\title{
Design and Control of a Cable-Driven Articulated Modular Snake Robot
}

\author{
Peter Racioppo
}

Thesis submitted to the faculty of the Virginia Polytechnic Institute and State University in partial fulfillment of the requirements for the degree of

\author{
Master of Science \\ In \\ Mechanical Engineering \\ Pinhas Ben-Tzvi, Chair \\ Lei Zuo \\ Alfred L. Wicks \\ December 11, 2017 \\ Blacksburg, Virginia
}

Keywords: robot dynamics; mobile robots; modeling; force control 


\title{
Design and Control of a Cable-Driven Articulated Modular Snake Robot
}

\author{
Peter Racioppo
}

\begin{abstract}
This thesis presents the design and control of a cable-actuated mobile snake robot. The goal of this research is to reduce the size of snake robots and improve their locomotive efficiency by simultaneously actuating groups of links to fit optimized curvature profiles. The basic functional unit of the snake is a four-link, single degree of freedom module that bends using an antagonistic cable-routing scheme. Elastic elements in series with the cables and the coupled nature of the mechanism allow each module to detect and automatically respond to obstacles. The mechanical and electrical designs of the bending module are presented, with emphasis on the cable-routing scheme, key optimizations, and the use of series elastic actuation. An approximate expression for the propulsive force generated by a snake as a function of its articulation (i.e. the number of links it contains divided by its body length) is derived and a closed-form approximation for the optimal phase offset between joints to maximize the speed of a snake is obtained by simplifying a previous result. A simplified model of serpentine locomotion that considers the forces acting on a single link as it traverses a sinusoid is presented and compared to a detailed multibody dynamic model. Control strategies for snake robots with coupled joints are developed, along with a feedback linearization of the joint dynamics. Experimental studies of force control, locomotion, and adaptation to obstacles using a fully integrated prototype are presented and compared with simulated results.
\end{abstract}




\title{
Design and Control of a Cable-Driven Articulated Modular Snake Robot
}

\author{
Peter Racioppo
}

\section{GENERAL AUDIENCE ABSTRACT}

This thesis presents the development of a cable-driven snake robot, with the goal of decreasing the size and mass of these devices and increasing their efficiency. Snake robots have potential applications in exploration and manipulation in cluttered or confined environments. The cable transmission system presented in this thesis allows for multiple links in a snake robot to be actuated simultaneously, allowing for increased articulation in a robot of fixed size and mass. Serpentine locomotion, in which a sinusoidal wave is propagated down the robot's length, is a silent and energy-efficient mode of transportation, widely employed in the animal kingdom. Snake robots achieve serpentine locomotion by driving their joints sinusoidally, with adjacent joints moving asynchronously, with the time lag between joints set by the value of a phase offset. An expression for the optimal phase offset to maximize forward velocity is derived by simplifying a previous result from the literature. An approximation of the dynamics of serpentine locomotion for a snake traveling at constant velocity is then derived, and this model is used to obtain an approximate limiting expression for the propulsive force generated per link as a function of the number of links in the snake. Methods to control a snake composed of coupled linkages are explored and the mechatronic design of a fully integrated prototype is presented. Experiments on force control, locomotion and turning, and detection and interaction with obstacles using the prototype are then described. 


\section{Design and Control of a Cable-Driven Articulated Modular Snake Robot Peter Racioppo}

Thesis Research Committee:

Pinhas Ben-Tzvi, Associate Professor of Mechanical Engineering, Associate Professor of Electrical and Computer Engineering, Virginia Polytechnic Institute and State University, Committee Chair

Lei Zuo, Professor of Mechanical Engineering, Virginia Polytechnic Institute and State University, Committee Member

Alfred L. Wicks, Associate Professor of Mechanical Engineering, Associate Professor of Electrical and Computer Engineering, Virginia Polytechnic Institute and State University, Committee Member 


\begin{abstract}
ACKNOWLEDGEMENTS
First of all, I want to thank my parents for always being there for me. I faced many unforeseen difficulties over the last two years, and I couldn't have done it without them.

I would also like to thank my advisor, Professor Ben-Tzvi, for motivating me to push myself in research and for encouraging me to pursue my own topics of interest. Thanks to him and the other members of the Robotics and Mechatronics Lab, I have become a better researcher and gained a new outlook on how to direct my future efforts. I want to thank the members of the lab who lent a hand with my project at one point or another. In particular, thanks to Prashant Kumar and Adam Williams for discussions on control, electronics, and more or less every aspect of my research. Thanks also to Vinaykarthik Kamidi for tips on design and other subjects, and Eric Refour and Hailin Ren for help with the electronics.
\end{abstract}




\section{TABLE OF CONTENTS}

ACKNOWLEDGEMENTS ........................................................................... iv

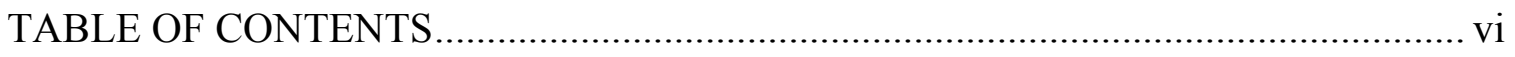

LIST OF FIGURES ..................................................................................... viii

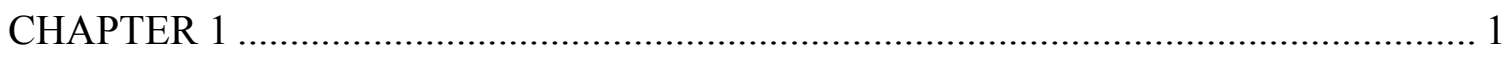

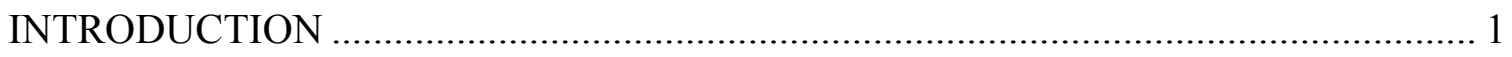

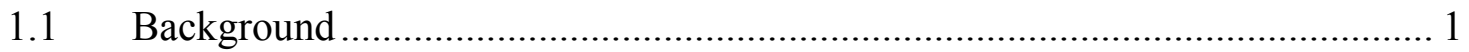

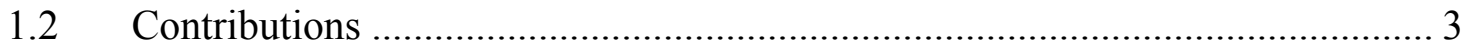

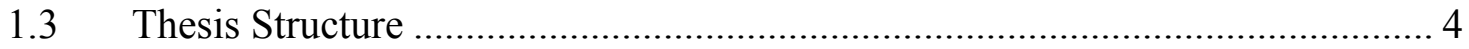

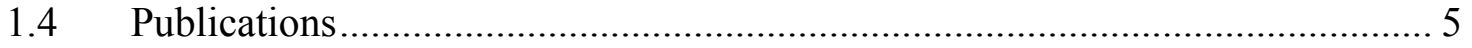

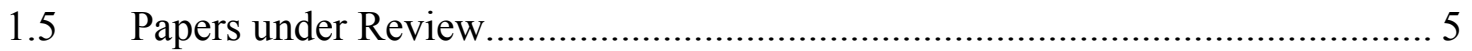

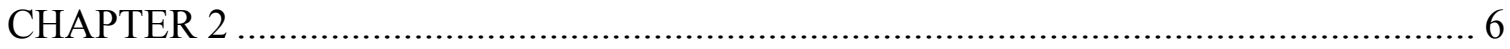

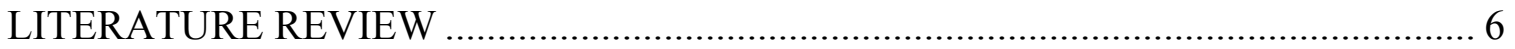

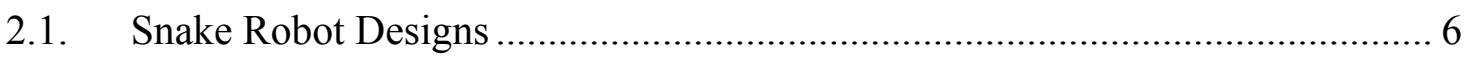

2.2. Modeling and Control of Snake Robots................................................... 9

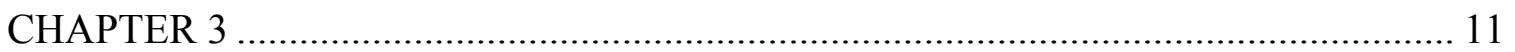

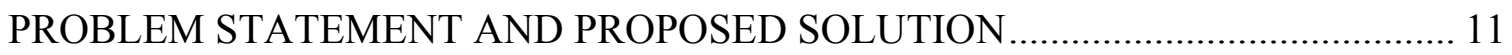

3.1. Motivations and Analysis of Related Research Problems .............................. 11

3.2. Research Objectives and Hypothesis ......................................................... 11

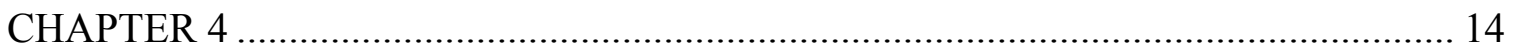

DESIGN OF THE CABLE-DRIVEN SNAKE ROBOT ........................................... 14

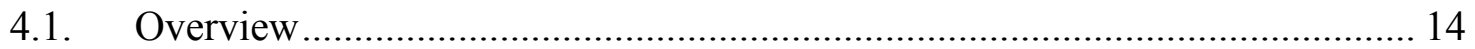

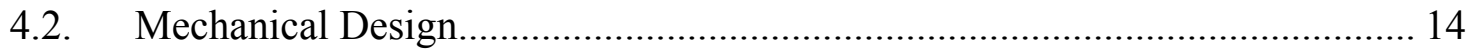

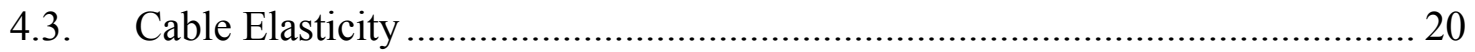

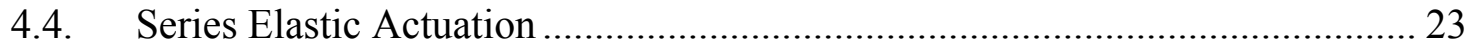

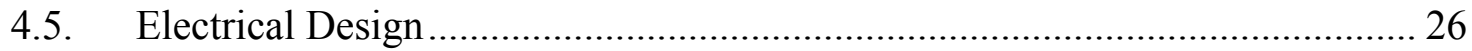

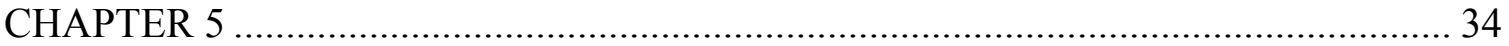

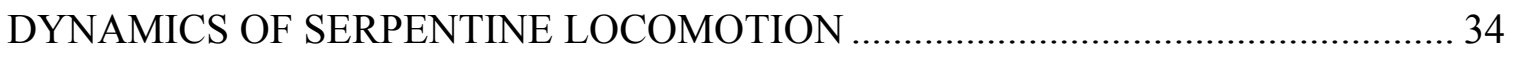

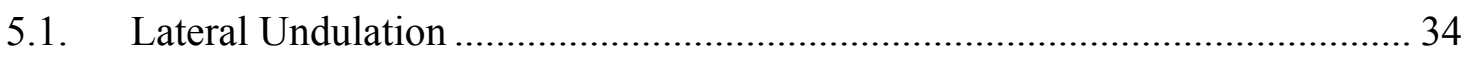

5.2. Derivation of an Approximation for the Optimal Joint Phase Offset .............. 34

5.3. Multibody Dynamic Model.......................................................................... 41 


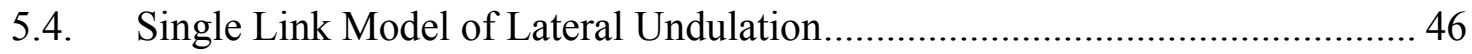

5.5. Bounds on Approximations by Discrete Linkages ........................................... 50

5.6. Online Estimates of Friction Coefficients......................................................... 51

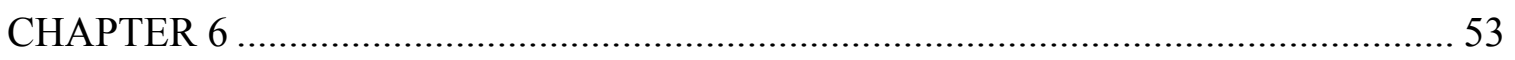

CONTROL OF A KINEMATICALLY CONSTRAINED SNAKE ROBOT .................. 53

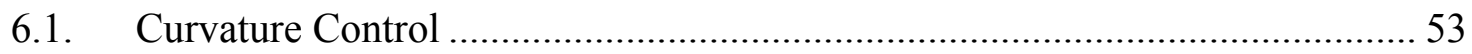

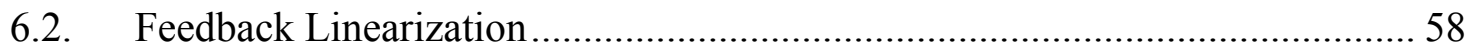

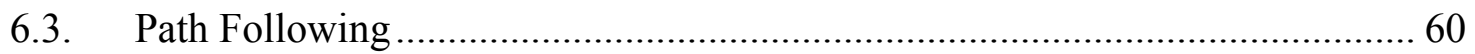

6.4. Simulation Study 1: Phase Offset Optimization ................................................. 63

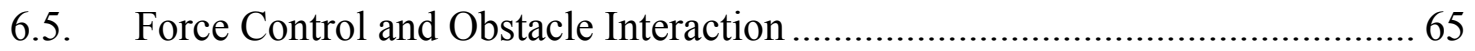

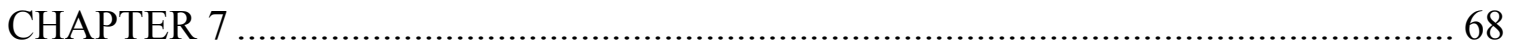

USE AS A MODULAR RECONFIGURABLE PLATFORM ……………………….... 68

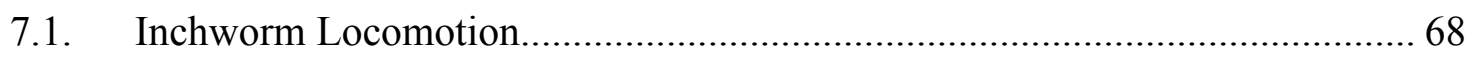

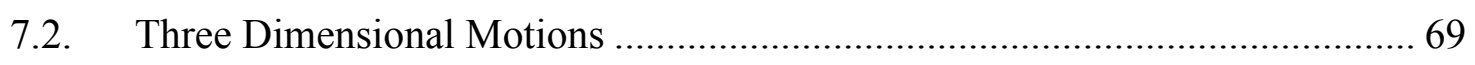

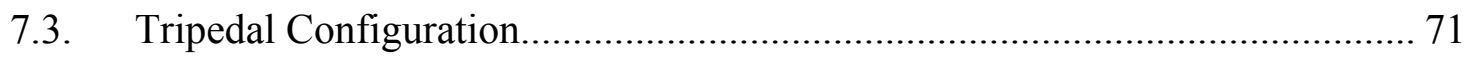

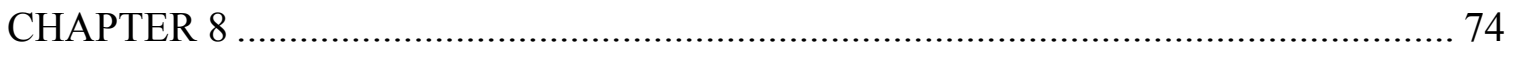

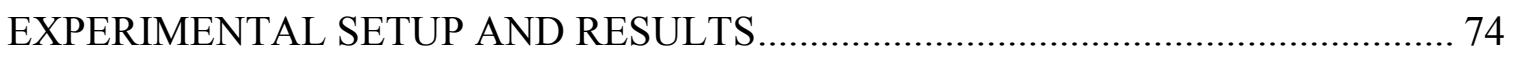

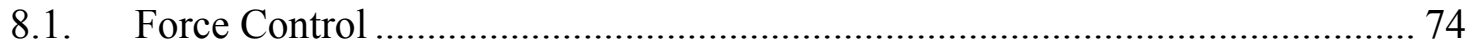

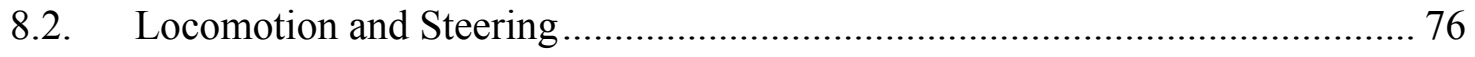

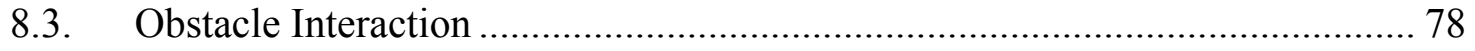

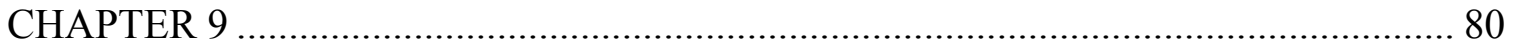

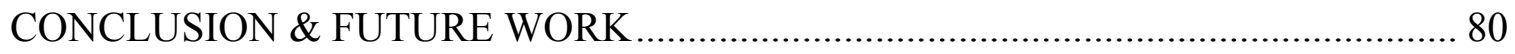

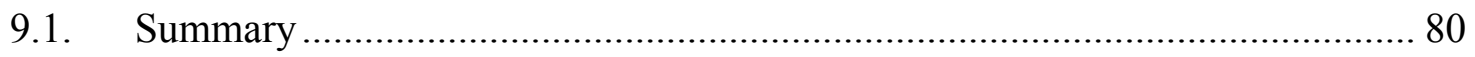

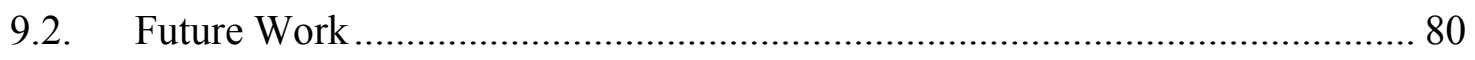

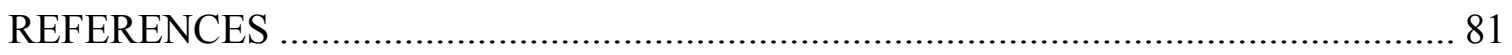




\section{LIST OF FIGURES}

Fig. 1. (a) Mechanical design of a four-link bending module near full extension.

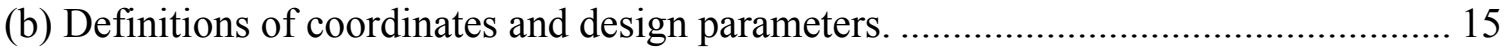

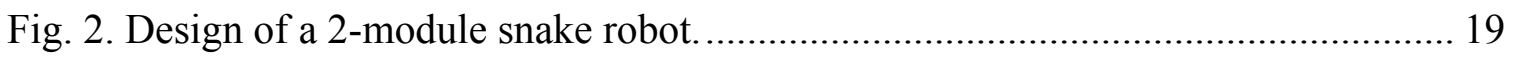

Fig. 3. Simplifed Cabling Schematic ................................................................... 20

Fig. 4. Illustration of conformation to obstacles, due to the mechanism's cabling. ......... 22

Fig. 5. Experimental measurements of joint angles after forced displacement of Link 2. 22

Fig. 6. Single-link model of series elastic actuation in the bending mechanism............. 24

Fig. 7. Step response of the closed-loop, single-link SEA model................................ 25

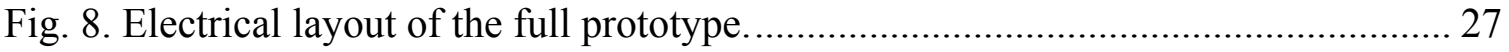

Fig. 9. (a) A single module, showing: (1) Actuation link, containing the servomotor. (2) A semicircular link. (b) Two-module snake robot prototype, showing (3) Head module,

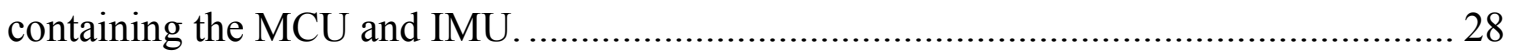

Fig. 10. Straight Wiring Scheme.............................................................................. 29

Fig. 11. Simulations of Cable Displacement (a) Cable Displacement / Link Length, (b)

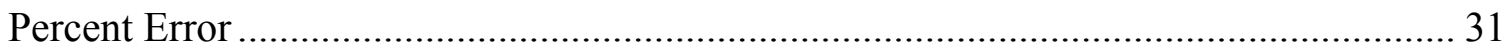

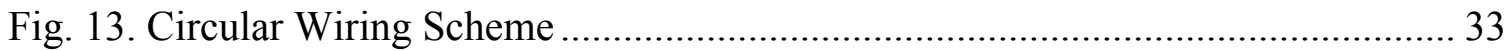

Fig. 14. Comparison of the three approximate expressions for $\delta_{\text {opt }}$, alongside the numerical solutions, for $N=3$ to 50 .

Fig. 16. Comparison of the full multibody dynamic model (black) with the single-link model (light) at various oscillation frequencies for (a) $\alpha=1 \mathrm{rad}$ and (b) $\alpha=0.5 \mathrm{rad}$..... 49 Fig. 17. Measurement of lateral friction coefficient, as errors are introduced into estimates of the snake's speed in the normal direction. 
Fig. 18. Simulation of Snake Locomotion: (a) Sum of Joint Angular Displacements, (b) Motor Torques 55

Fig. 19. Trajectory of an 8-link snake robot in a 120 second simulation. ...................... 56

Fig. 20. Forward speed of an 8-link snake robot in a 120 second simulation. 57

Fig. 21. Simulated steady-state speeds of two 2-DOF snake robots, one with three links (dotted line) and one with eight (solid line), as $a$ and $\omega$ are varied. 58

Fig. 22. Steady-state speed for a 2-module snake robot as estimates of external forces are varied between $0 \%$ and $140 \%$ percent of the actual values. 60

Fig. 23. Path following under a disturbance 62

Fig. 24. Optimal phase-offset vs. lateral friction coefficient. 64

Fig. 25. Steady state speed vs. joint angle ratio. 65

Fig. 26. The complete control scheme for the cable-driven snake robot.

Fig. 27. Position and forward speed of an 8-link snake robot. 70

Fig. 28. Modeled Moments of Inertia of the Bending Module..... 71

Fig. 29. Force control experiments for (A) Straight configuration $\left(\theta_{1}=0^{\circ}\right)$ and (B) Curved configuration $\left(\theta_{1}=30^{\circ}\right)$. 75

Fig. 30. Snapshots of the snake robot executing a counterclockwise turn, showing a half period of oscillation between (A) and (B). The black marker in the top left corner provides a point of reference.

Fig. 31. Trajectories of the front link of the snake robot over approximately four oscillation periods, for various values of $\theta_{0}$. (Positive values correspond to clockwise turning). 
Fig. 32. Trajectories of the back link of the snake robot during interaction with a circular obstacle. Initial position is denoted by two concentric circles at $(0,0)$. 79 


\section{CHAPTER 1}

\section{INTRODUCTION}

\subsection{Background}

Snake robots, like their biological counterparts, consist of serial-linkages of segments, and locomote primarily by pushing against external objects using undulatory waves [1]. These mechanisms are typically highly articulated, allowing them to change their body shape to manipulate external objects and operate in a wide variety of environments [2]. The lateral undulation gait, more commonly called serpentine or slithering locomotion, allows a snake to generate propulsive forces by propagating sinusoidal waves along its length, provided that it makes anisotropic frictional contact with the ground, with higher coefficients of friction normal to its body length than along it body length [3],[4]. Unlike wheeled or legged robots, a snake robot may thus engage any part of its body to locomote, making it well suited to cluttered or confined spaces likely to be encountered in e.g. search and rescue missions or inspection tasks [5]. Undulatory locomotion is also a notably energy-efficient and silent means of transportation, and is thus appropriate for use in a variety of prolonged or low-speed activities [4]. Snake-like robots have also found use as manipulators, including in surgical settings, where their ability to conform to narrow channels such as the throat, the ear canal, and difficult-to-reach spaces behind internal organs may make them key surgical instruments [6],[7].

Modern mobile snake robots typically feature about an order of magnitude less articulation per length than biological snakes [8], and rely on individual motors to drive each joint, as opposed to the complex, interwoven web of musculature used in nature. 
Direct drive mechanisms have been used in a wide variety of these robots, including the first snake robot ACM III [9]; the robots developed at CMU by Choset et al, which demonstrated pole climbing and obstacle traversal [10]-[12]; snakes with active wheels or treads such as the ACM-R4 and OmniTread OT-8, [13],[14]; the modular selfreconfigurable MTRAN and PolyBot robots [15],[16]; and the ACM R5 [17], which demonstrated locomotion in water. With fewer joints per length than biological snakes, robotic snakes must drive their joints at greater amplitude to approximate a given curvature profile, and are limited by the mass and cross-sectional area requirements of their actuators. Snake-like manipulators have achieved smaller cross-section than mobile snake robots through the use of axially routed cables, which allow actuators to be located away from the joints of the end-effector. Notable examples include the CardioARM robot, intended for heart surgery, which features cylindrical links of only $10 \mathrm{~mm}$ diameter actuated by internally routed cables [6], and the continuum manipulator of Ouyang et al, which consists of a three-segment, super-elastic nitinol rod backbone actuated by axial cables [18]. These systems achieve extremely small scale and high articulation, but require external pulleys and actuators, and thus are not suited for use in mobile robots. To address these issues, we have developed a multi-link bending module that can simultaneously actuate a group of four links using agonist-antagonist cable pairs routed around a multi-radius pulley. This modular design is intended to support two design paradigms, either (1) serving as the basic functional unit in a highly articulated snake robot or (2) allowing snake robots with fewer degrees of freedom (DOFs) to respond passively to obstacles, using inbuilt properties of the mechanism. 


\subsection{Contributions}

This thesis makes the following contributions to the snake robot literature:

- Design and integration of the first cable-driven mobile snake robot.

- Simplification of a previous expression for the optimal joint-angle phase offset to maximize the speed of a snake robot.

- Development of a simplified dynamic model of serpentine locomotion.

- An analysis of how the propulsive force produced by a snake robot depends on its level of articulation (i.e. how many links it contains per body length).

- Simulation experiments on serpentine locomotion using a detailed multibody dynamic model.

- Development of control strategies for a snake robot with kinematically coupled joints.

- Analyses of a novel cable-actuated mechanism, including computations of cable displacements and work on design synthesis, comparative analysis of related mechanisms, and initial work on the use of this module as a self-reconfigurable robotic platform.

- A partially linearizing feedback controller for this mechanism that accounts for cable dynamics.

- Modifications to a path following algorithm for a snake robot.

- A method for online measurement of friction coefficients during slithering. 


\subsection{Thesis Structure}

The thesis is organized as follows: Chapter 2 reviews previous designs of mobile snake robots and snake-like manipulators, as well major developments in the modeling and control of these systems. Chapter 3 outlines outstanding problems in the field and proposes a novel design paradigm to address these issues. Chapter 4 presents the mechanical and electrical design of the cable-driven snake robot developed in this thesis. The cable routing scheme of the present design is also explored and compared with alternative mechanisms. Chapter 5 presents a simplification of a previous expression for the optimal joint phase offset to maximize the speed of a snake robot and discusses a simplified "single-link model" of lateral undulation, intended to capture the central features of this locomotion style. A detailed dynamic model is also developed in this section, and compared with the simplified model. Chapter 6 develops a control scheme for the robot and includes discussions of feedback linearization, path following, and obstacle interaction. Additional uses of the module as a modular self-reconfigurable robotic platform are discussed in Chapter 7. Chapter 8 presents experiments on locomotion and obstacle interaction using a two-module prototype. Concluding remarks and plans for future work are discussed in Chapter 9. 
Disclosure: The material contained in this thesis resulted in the following publications.

\subsection{Publications}

P. Racioppo, W. Saab, and P. Ben-Tzvi, "Design and analysis of a reduced degree of freedom modular snake robot," International Design Engineering Technical Conferences \& Computers and Information in Engineering Conference, vol. 5B, pp. V05BT08A009, 2017.

P. Racioppo and P. Ben-Tzvi, "Modeling and control of a cable driven modular snake robot," IEEE Conference on Control Technology and Applications, pp. 468 - 473, 2017.

\subsection{Papers under Review}

P. Racioppo and P. Ben-Tzvi, "Design and Control of an Articulated Modular Snake Robot," Transactions on Mechatronics. Submitted Dec. 10, 2017.

W. Saab, P. Racioppo, A. Kumar, and P. Ben-Tzvi, "Design of a Miniature Modular Inchworm Robot with an Anisotropic Friction Skin," Submitted to Robotica, Nov. 17, 2017. 


\section{CHAPTER 2}

\section{LITERATURE REVIEW}

This section provides an overview of prior research into the design of snake-like mechanisms, and empirical studies of locomotion in biological snakes. Essential properties of snake robots are described, including friction characteristics, types of hyperredundant structures, the use of direct-drive versus cable-actuated systems, and compliance. The design motivation for the bending mechanism and general requirements for its operation are then discussed.

\subsection{Snake Robot Designs}

Early observational studies of serpentine motion in snakes, such as those of W. Mosauer and J. Gray, provided foundational insight into the basic mechanism by which ground friction in the transverse and lateral directions of a snake's body propels it forward, and the importance of the snake's curvature profile for locomotion [19],[3]. Curvature in biological snakes is controlled by an intricately arranged web of axial muscles, which, as shown by J. Gasc, produce angular displacements between adjacent vertebrae in the range of 10 to 20 degrees [20]. Significant angular displacements in biological snakes thus must involve underactuated groups of vertebrae.

Work on snake robots was initiated by S. Hirose's development of ACM III, which demonstrated serpentine motion on a flat plane [21]. Hirose's design employed passive wheels to produce the anisotropic ground friction characteristics necessary for forward locomotion. Passive wheels are a common means of producing anisotropic ground friction 
in snake robots, but may fail to make effective contact in cluttered or irregular environments. In biological snakes, anisotropic friction is instead produced by the geometrical and mechanical properties of scales [22]. B. Jayne studied the mechanical properties of the skins of six snake species and noted correlations between skin characteristics and locomotion style [23]. Marvi et al., studied active control of scales in corn snakes and developed the two-link inchworm robot ScalyBot, to study the effects of actively adjusted scales on locomotion, on flat and inclined planes [24]. Grooves, static scales, and friction skins have also been widely used to incorporate the desired friction characteristics directly into the construction of a snake robot's outer shell, as in the 3D printed scales employed by the Scaled Snake Robot, or the bristle-covered fabric used in the MMIR robot [25],[26].

The cyclical curving motion executed during serpentine motion may be achieved mechanically using either serpentine or continuum type mechanisms. Serpentine designs, which are defined by their serial chain of rigid links, may closely approximate continuous curves, provided a sufficient level of articulation, with the benefit of robust sub-structures convenient for housing actuation. Continuum designs, which make use of a compliant backbone to achieve continuous bending, eliminate the need for discrete joints, and are thus well suited to small-scale applications. However, continuum mechanisms exhibit problematic sagging under loading and pose difficulties to conventional means of modeling and sensing. Hybrid approaches include the use of multiple backbones [18], interleaved flexible and rigid links [27], and spherical joints situated around an elastic core [28].

The majority of snake robots in the literature are serpentine type mechanisms and employ direct drive at each link to maximize joint torque and drive stiffness. This design 
choice is incorporated by a wide variety of snake robots, including the unified modular snaked robot which features compact revolute joints to enable gripping and climbing [10]; the Wheeko robot [5], constructed to investigate serpentine motion on a flat plane; the Kulko robot, designed to investigate interaction with obstacles [5]; the reconfigurable PolyBot [16]; and the ACM R5 [17], which demonstrated locomotion in water with the use of fins mounted on each link. Direct drive is also widely used in snake robots which employ active wheels or treads, such as the ACM-R4 and OmniTread OT-8, respectively [29],[13].

Cable-driven actuation in both serpentine and continuum type robots has primarily been pursued in the context of manipulators, as a means of decreasing mass and cross sectional area requirements by allowing the mechanism's actuators to be located away from the joints they actuate [7]. Ota et al. built and tested the serpentine-type CardioArm robot consisting of a series of cylindrical links only $11 \mathrm{~mm}$ in radius, and actuated by internal cables [6]. Ouyang et al. developed a continuum-type robot composed of a three-segment, super-elastic nitinol rod backbone with concentric discs used for cable routing [18]. Cable actuation schemes allowed these robots to be small and highly articulated, but required the use of multiple externally driven pulleys.

In self-contained mechanisms, where space is limited, the use of cabling requires that pulley and actuation systems be made as compact as possible. Antagonistic cable pairs and multi-radius pulleys may be used in this context to consolidate the actuation of multiple links. A variety of mechanisms which use antagonistic pairs of cables to generate joint motion have been designed, including Hillbery and Rolamite joints, which employ cylindrical rollers actuated by antagonistic bands to produce low-friction, planar bending [30]. A number of cable actuated robotic hand designs have incorporated antagonistic cable 
pairs, including the DLR, a versatile, variable stiffness robotic hand, and the PISA/IIT SoftHand, which exploits underactuated, compliant cabling to produce adaptive grasping behavior [31],[30]. A disadvantage of straight-line routing of antagonistic cables in bending mechanisms is that displacements in antagonistic cable segments may exhibit nonlinearities during bending, depending on the details of the routing scheme. This challenge is discussed in more detail in Section 4.

Compliant elements may be included in snake robots to filter high frequency disturbances, shield motors, and enable automatic conformation to obstacles, highly desirable properties for robots that engage their entire bodies during locomotion [32], [33]. Rollinson et al. developed a snake robot that employed series elastic actuators (SEAs) consisting of conical rubber elements placed at each joint, and demonstrated threedimensional gaits and climbing of vertical poles [34]. Ahmed and Billah designed a compliant backbone snake robot incorporating flexible tendons made from electroactive polymer materials [35].

\subsection{Modeling and Control of Snake Robots}

Shigeo Hirose [1] is widely credited with pioneering the field of snake robotics with his development of ACM III, the first snake robot, and his discovery of the "serpenoid curve," an approximation for the shape of a biological snake performing serpentine motion. Further work on mathematical descriptions of serpentine locomotion was conducted by Shugen Ma [36], who compared the locomotive efficiency of various curvature profiles and developed an alternative to the serpenoid curve. Other approaches to achieving planar locomotion include Chernousko's study of a 3-link snake robot, in which the joint angles are made to perform "elementary motions" from which more complex gaits can be built 
[37]. Liljebäck et al. demonstrated a path following control scheme and waypoint guidance in a snake robot approximating the serpenoid curve, both in simulation and experimentally [38]-[39]. Liljebäck et al. also proved that anisotropic ground friction is a necessary condition for controllable locomotion, developed a simplified model of serpentine locomotion consisting of only translational motions, and tested jamming resolution schemes during interaction with obstacles [40]-[41]. Liljebäck et al. developed the Kulko robot to test these advances experimentally. Kulko does not make anisotropic frictional contact with the ground, and can thus be used as a control for obstacle interaction strategies [5]. Optimization of snake robot design parameters has also been a major focus of research, particularly with the aim of maximizing the robot's average speed [37],[2]. 


\section{CHAPTER 3}

\section{PROBLEM STATEMENT AND PROPOSED SOLUTION}

\subsection{Motivations and Analysis of Related Research Problems}

Lessons derived from the literature highlight several main factors that drove the design of the cable-driven snake robot.

- Biological snakes display small maximum angular displacements between adjacent links and continuous curvature during lateral undulation. Groups of links can thus be approximated with underactuated, curving structures. Biological snakes also feature highly coupled actuation, as a means of decreasing space requirements.

- Mobile snake robots are typically actuated with bulky, direct-drive motors. Snake-like manipulators tend to actuate multiple links with a single motor via cabling, allowing link mass and cross sectional area to be reduced and avoiding the problems associated with direct drive.

- Cable-driven serpentine mechanisms are often limited by the size of pulley actuation systems, which may be made smaller using antagonistic cable pairs and multi-radius pulleys.

- Straight routing schemes may produce nonlinearities in the displacement of antagonistic cable pairs. This problem can be avoided by routing cables along circular surfaces.

\subsection{Research Objectives and Hypothesis}

In light of the foregoing observations about the state of the art in snake robotics, this thesis hypothesizes the following: 
- Efficiency - Actuating kinematically coupled linkages may increase the average propulsive force produced by each degree of freedom in a snake robot and minimize the drag force by allowing the robot to better approximate desired curvature profiles.

- Size and Weight - Actuating multiple joints simultaneously using cables may allow for the cross-sectional area and weight of snake robots to be decreased considerably, by removing the need for large and heavy actuators at each joints.

- Series Elastic Actuation - Series elastic actuators can provide a robot with force control and impulse filtering capabilities, which are particularly useful in a snake robot, which must contact the environment along its entire body length. Extension springs are lighter, smaller, and easier to manufacture than specialized torsional elastic elements. Cable-actuation provides a natural means of implementing series elasticity without the use of torsional elements.

- Obstacle Interaction - Actuating multiple joints with elastic cables naturally results in local, uncontrollable behavior at the joints, which may provide a snake robot with a means of automatically conforming to external obstacles without the use of actuators at every joint.

- Simplicity - A snake robot can locomote and steer in the plane with as few as two degrees of freedom. Constructing such a snake robot from two, highly articulated modules would allow the robot to approximate optimal curvature profiles, and thus be highly efficient, without the need for many degrees of freedom. The decrease in 
redundancy can be offset by the automatic behavior provided by the series elasticity.

- Force Control - Adding elasticity in series with cables may allow a snake robot to control the force it exerts on its environment by measuring cable displacement. In turn, cable displacements can be related to joint deflections by the kinematic constraints. Force control may allow a snake robot to sense when it contacts obstacles during locomotion and perform other useful functions such as grasping or pushing off external objects.

- Modular Reconfigurability - Snakes are well suited to at least two distinct types of activity: navigating confined or irregular terrain and manipulating objects. Designs of this type can thus potentially act as modular self-reconfigurable units that act independently to move in difficult environments and then interact at a target to perform complex manipulation functions. 


\section{CHAPTER 4}

\section{DESIGN OF THE CABLE-DRIVEN SNAKE ROBOT}

\subsection{Overview}

This section presents the design of the snake robot, first discussing the mechanical design and cable-routing of an individual bending module, then describing the use of series elastic actuation and the resulting obstacle-interaction behavior, and finally outlining the electronic design and implementation of a preliminary two-module prototype.

\subsection{Mechanical Design}

This section describes the mechanical design of the Planar Bender, shown in Fig. 1, and the mechanism's cable-routing scheme.

Each bender is a modular, single-DOF unit, composed of a serial chain of $N$ rigid links connected by parallel revolute joints. The mechanism is actuated by an antagonist-pair tendon transmission system, in which opposing cables coordinate their contraction and elongation phases to avoid interfering with each other's motion. The $N-1$ cable pairs are routed along the circular exteriors of the links in circular grooves, which prevent lateral slippage and cable interference. Routing cables along circular paths rather than routing in 


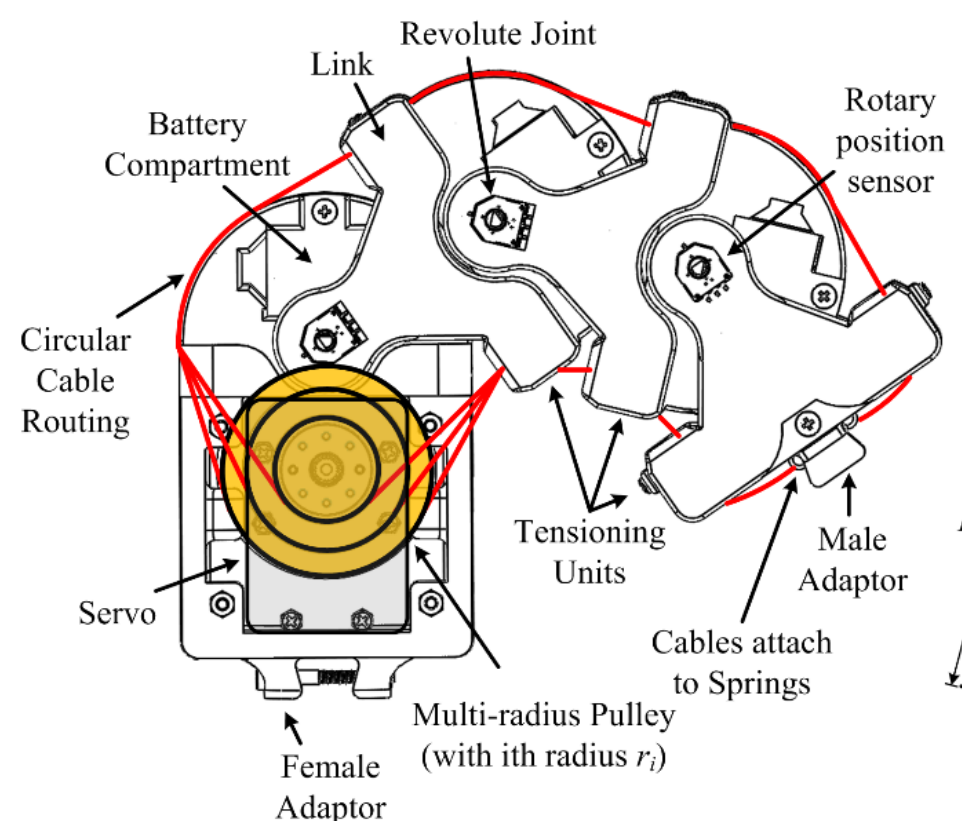

(a)

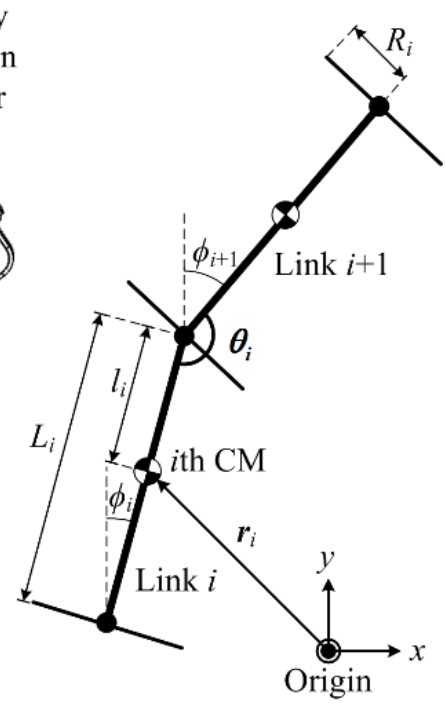

(b)

Fig. 1. (a) Mechanical design of a four-link bending module near full extension. (b) Definitions of coordinates and design parameters.

straight lines from link to link constrains the cable displacements on opposite sides of the mechanism to be equal and opposite in value. Each cable pair attaches to a single link and terminates on a multi-radius pulley, located in the actuation unit. Rotating the pulley displaces each cable segment, inducing a curvature profile in the mechanism that depends on the ratios of the pulley radii. The cable pair that connects to the $n$th link is routed along all of the $n-1$ intermediate links, resulting in kinematic coupling, since the cable displacement that occurs at the $n$th link is influenced by the rotation of those between it and the base. The pulley is driven with a high-torque servomotor, which is inserted into the base compartment via a detachable side-wall, to ensure proper alignment of the pulley channels with the exterior cable grooves. Given the pulley radii, a single rotary encoder on the servomotor shaft is sufficient to determine the full bending profile of the mechanism. 
Each of the driven links is hollow and low mass, providing extra space for electronics and on-board batteries.

Cable tensioning, which is necessary to prevent backlash and hysteresis-nonlinearities, and ensure that the mechanism is in a straight configuration at equilibrium, is accomplished using a sliding tensioning unit, which inserts into slots in laterally placed bars on the side of each link. Tightening screws on a tensioning unit forces it further into its slot, increasing the tension on the wires that pass underneath. These units also prevent cables from slipping normal to the link surfaces. Tensioning must be performed sequentially, starting with the link closest to the base, in order to ensure that the kinematic constraints consistent with a straight configuration are satisfied at each step.

Compliance is of particular importance in the context of a reduced-DOF snake, as it allows the robot to automatically conform and rebound from obstacles in the absence of individually actuated joints. Elastic elements also perform the vital task of filtering the high-frequency disturbances that arise during locomotion, which decreases the shock impulses experienced by the motor. Compliance in the Planar Bender is achieved with extension springs attached in series with each of the cables, removing the need for a specially designed torsional element, which would increase the size of the base compartment. Cable lengths at the home configuration are selected to produce a small extension in each of the springs, such that all of the springs remain in tension throughout bending.

The anisotropic friction characteristics necessary for locomotion are produced using a bristle-covered fabric whose friction characteristic depend on the orientation of the bristles relative to the ground plane. This friction-skin material produces different coefficients of 
friction in its lateral and transverse orientations, defined relative to the alignment of the bristles, and has already been characterized by the authors in the context of inchworm locomotion [26]. The small-scale structure of the friction skin is well suited to producing anisotropic friction on regular and even terrain, such as carpeted or tiled surfaces. During planar movement, the rigid structure of the bender ensures that every part of the friction skin is on a level plane and remains uniformly engaged. The skin has the property that the coefficient of friction that opposes forward motion is lower than the corresponding coefficients for both backward and lateral motion. The combination of these two properties is ideal for producing forward locomotion, as it both supports the serpentine gait and prevents back-slipping.

The Planar Bender is designed to be a self-contained experimental platform for investigating a variety of modes of snake locomotion. To that end, each module includes a male-female interface as a simple means of connecting modules in series, while the curvature profile of the mechanism may be modified at any time by replacing the pulley. Connecting multiple Planar Benders in series produces a "Planar Slitherer," displayed in Fig. 2, two modules being the minimum required for serpentine locomotion and turning. Modifying the curvature of each Planar Bender sinusoidally and out of phase with the adjacent module produces forward locomotion. Turning may be achieved by oscillating the benders asymmetrically, such that the maximum angular displacements on the left and right sides are unequal, resulting in a moment which rotates the snake. The ability of each module to independently execute a full bending motion allows for the addition of components with other functions, without the loss of slithering functionality. Modules that enable additional degrees of freedom, such as inchworm-like linear progression or rotation 
about the snake's principal axis, may be linked in series with bending modules to enable both planar slithering and three-dimensional motion, as shown in Fig. 2.

Rotating the multi-radius pulley by an angle $\theta_{P}$ induces a curvature profile in the mechanism, given in terms of the angle of the $i$ th joint $\theta_{i}$ (where $i=1$ at the actuation module), by the relation:

$$
\theta_{i}=\left(\frac{r_{i}}{\sqrt{l_{i}^{2}+R_{i}^{2}}}-\frac{r_{i-1}}{\sqrt{l_{i-1}^{2}+R_{i-1}^{2}}}\right) \theta_{P}
$$

where $r_{i}$ is the $i$ th pulley radius, $R_{i}$ is the radius of the $i$ th semicircular link, and $l_{i}$ is the half-length of the $i$ th link, as illustrated in Fig. 1(b). The pulley radii can thus be optimized to fit a given static curvature profile. Progressive bending along the mechanism requires a monotonic increase in the corresponding pulley radii: $r_{1}<r_{2}<\ldots<r_{n}$, which limits the number of links that the mechanism can accommodate for a given cross sectional area. A single module cannot bend in multiple directions at once, so a chain of $n$ modules can have at most $n-1$ inflection points. The multi-radius pulley is rotated with a high-torque servomotor equipped with a rotary encoder, which together with the kinematic constraints in (1), is sufficient to measure the full body-shape of the module, provided that the extension springs are unextended. Rotary position sensors located at each joint measure displacements in the springs, for use in the obstacle interaction procedure described in Sec. 6.

As can be seen in Fig. 3, the tension forces acting on link $i$, in the cables above and below that link, occur in equal and opposite pairs. For link $i$, the only cable forces that generate a moment in joint $i$ are the pair acting between links $i-1$ and $i$. The joint torques of an $N$-link module due to a motor torque $\tau_{\mathrm{M}}$ can be computed by solving recursively from 
the end of the mechanism to its base, so that the cable dynamics of the module are fully defined by a set of $N$ coupled algebraic equations:

$$
\tau_{\mathrm{M}}=\sum_{i=1}^{N-1} F_{i} r_{i}, \tau_{i}=\sum_{j=i}^{N-1} F_{j} R_{j}
$$

where $F_{i}$ is the $i$ th cable tension and $\tau_{i}$ is the $i$ th joint torque [42]. The bottom of each link features wheels oriented parallel to the length of the zsnake, giving the robot's bottom surface the appropriate orientation-dependent friction coefficients. Adjacent modules can connect via a rigid male-female interface.

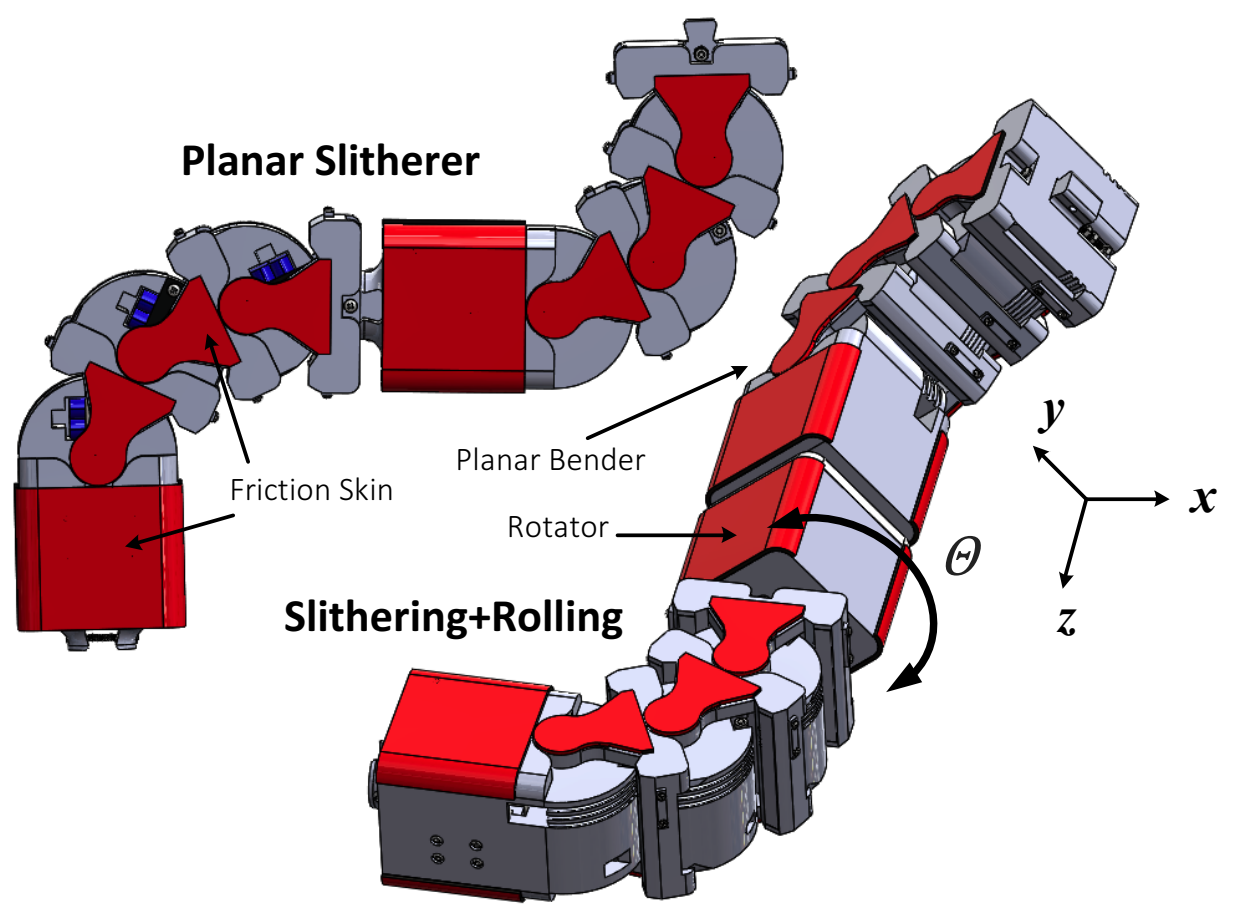

Fig. 2. Design of a 2-module snake robot. 


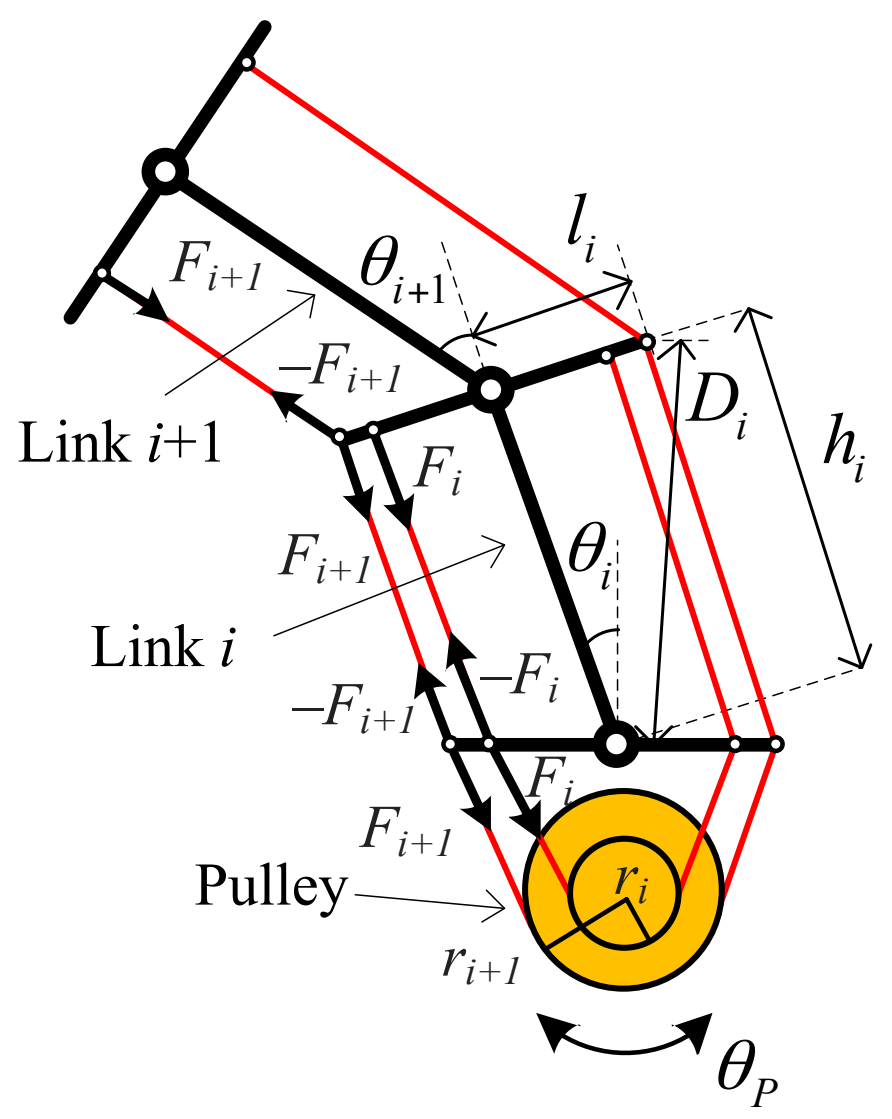

Fig. 3. Simplifed Cabling Schematic

\subsection{Cable Elasticity}

Elastic elements are commonly used in robotics applications as a means of low-pass filtering impacts, storing energy during locomotion, and enabling force control by mapping displacements to forces [43]. In snake robots, previous work on elastic elements includes the development of a torsional rubber elastic element by Rollinson et al, for use in a poleclimbing snake robot [44],[45]. In this section, we consider the use of extension springs placed in series with each cable. Extension springs are smaller and easier to implement than specialized torsional springs and are well suited to a cable transmission system. Here, we have chosen equal spring constants of approximately $500 \mathrm{~N} / \mathrm{m}$ each, selected in order to permit desired angular displacements under anticipated contact forces. 
During unimpeded motion, displacements in antagonistic cables are equal and opposite, so the springs in line with the cables are not engaged. However, if an external force is applied from one side of a link, the spring on this side of the link will extend and an angular displacement may be induced in the adjacent joints. Adjacent links will then experience angular displacements in the opposite direction, since no extra cable length has been provided for them, causing the mechanism to passively bend toward the obstacle, as shown in Fig. 4. In the same way, if links $n$ through $m$ are forcibly displaced, the $(m+1)$ th link must rotate to compensate for their rotation. If also obstructed, the $(m+2)$ nd must compensate for the remaining cable displacement, and so on:

$$
\sum_{i=n}^{m} \theta_{i}=-\sum_{j=m+1}^{N} \theta_{j}
$$

Each bending module will thus tend to automatically bend around obstacles with low radius of curvature and conform to obstacles with high radius of curvature. The relationship in (3) was tested in a prototype of the four-link mechanism described in Sec. 2, the results of which are shown in Fig. 5. After a sinusoidal forced displacement of Joint 1 beginning at about 7 seconds, Joint 2 compensates by moving in the opposite direction. A forced displacement in the opposite direction begins at about 27 seconds, but Joint 2 reaches an obstruction at about 35 seconds, after which Joint 3 must compensate for the remaining displacement in Joint 1. 


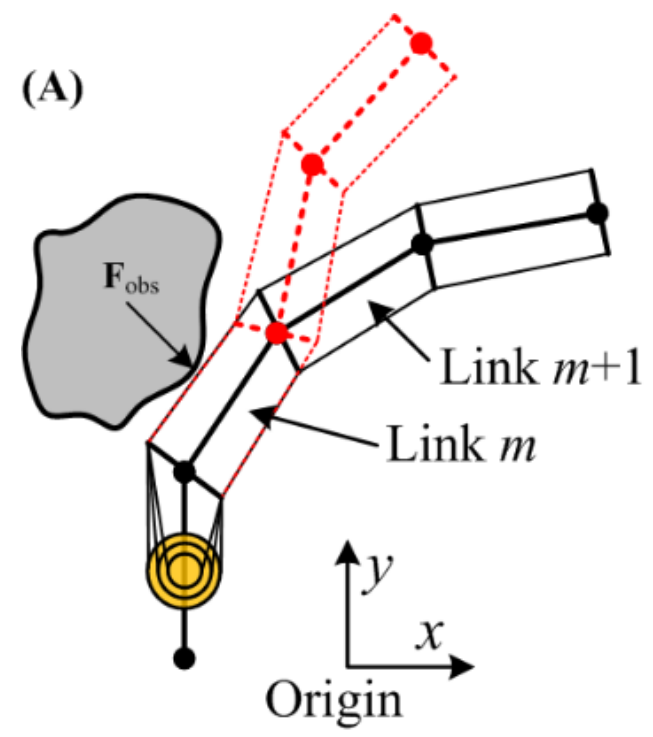

(B)

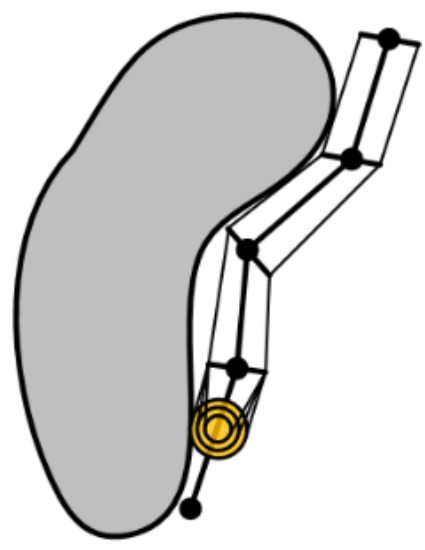

Fig. 4. Illustration of conformation to obstacles, due to the mechanism's cabling.

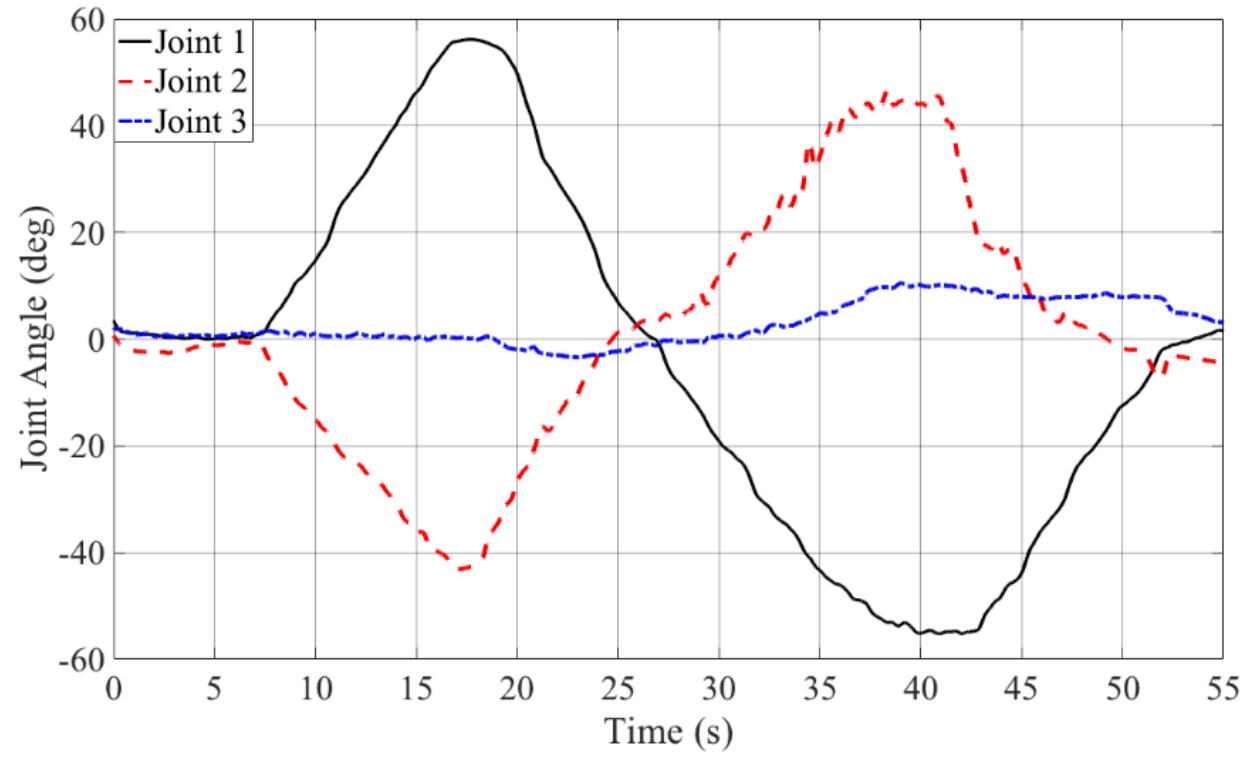

Fig. 5. Experimental measurements of joint angles after forced displacement of Link 2. 


\subsection{Series Elastic Actuation}

The cable-pair springs described in the previous section are always located between the motor shaft and external forces and can therefore function as a set of coupled series elastic actuators (SEAs). The full SEA system can be modeled by a system of $N$ differential equations in $N$ unknowns: $\left\{\begin{array}{c}\tau_{m}+\tau_{k}=J_{m} \theta_{m}, i \in[1, \ldots, N-1] \text {, where } \\ \tau_{i}=I_{i} \theta_{i}\end{array}\right.$ $\left\{\begin{array}{l}\tau_{k}=-\sum_{j=i}^{N-1} r_{j} k_{j}\left(r_{j} \theta_{m}-D_{j} \theta_{j}\right) . \text { Here, } \tau_{k} \text { is the "elastic torque" acting on the motor due to the } \\ \tau_{i}=\sum_{j=i}^{N-1} R_{j} k_{j}\left(r_{j} \theta_{m}-D_{j} \theta_{j}\right)\end{array}\right.$ displacements of the springs, and the $\tau_{i}$ are the torques that the springs cause at each of the mechanism's joints. This situation can be simplified by ignoring link inertia, reducing the set of $N$ differential equations to a single differential equation with $N-1$ algebraic equations. The system can be simplified further by considering a mechanism with only a single link, as in Fig. 6.

In this final simplification, considering only actuation torque $\tau_{m}$, elastic torque on the motor due to the springs $\tau_{k}$, and motor inertia $J_{m}$, the equation of motion for the motor is: $\tau_{m}+\tau_{k}=J_{m} \theta_{m}$. The elastic torque is the product of the pulley radius $r$ and the cable tension $F_{k}$ due to a spring extension $\Delta x$, with a spring constant $k: \tau_{k}=r F_{k}=-r k \Delta x$. The spring extension due to an angular displacement at the motor of $\theta_{m}$ and an angular displacement of the first joint of $\theta_{1}$ is approximately: $\Delta x=r \theta_{m}-R \theta_{1}$, (taking $D \approx R$ ). Thus, $\tau_{k}=-r k\left(r \theta_{m}-R \theta_{1}\right)$. The elastic torque at joint one is $\tau_{1}=-R F_{k}=R k\left(r \theta_{m}-R \theta_{1}\right)$, where $R$ is the width of a link, which implies that $\tau_{k}=-\frac{r}{R} \tau_{1}$. 


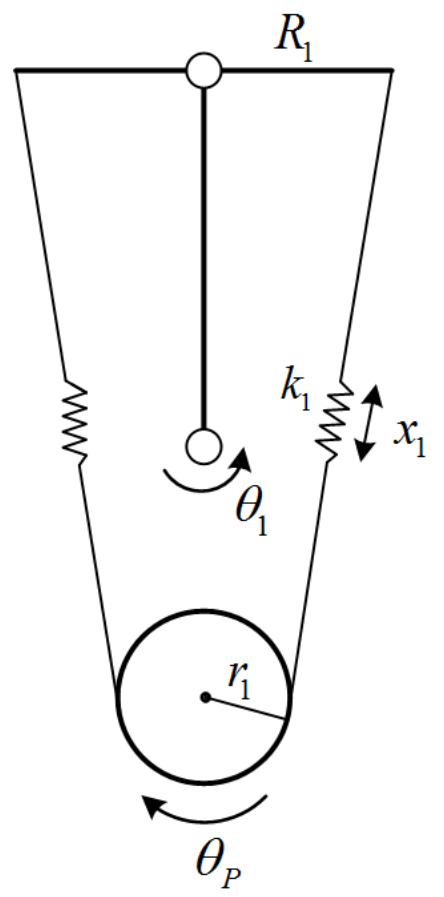

Fig. 6. Single-link model of series elastic actuation in the bending mechanism.

Substituting this expression into the equation of motion for the motor, we have that $\tau_{m}=\frac{r}{R} \tau_{1}+J_{m} \theta_{m}$, and taking a Laplace transform, $\tau_{m}=\frac{r}{R} \tau_{1}+J_{m} \theta_{m} s^{2}$. Solving for $\theta_{m}$ in the above expression for $\tau_{1}$ gives that $\theta_{m}=\frac{1}{r}\left(\frac{\tau_{1}}{R k}+R \theta_{1}\right)$, and substituting gives that $\tau_{m}=\frac{r}{R} \tau_{1}+\frac{J_{m}}{r}\left(\frac{\tau_{1}}{R k}+R \theta_{1}\right) s^{2}$. Collecting terms, we arrive at $\tau_{m}=\frac{1}{R}\left(r+\frac{J_{m}}{r k} s^{2}\right) \tau_{1}+\frac{R}{r} J_{m} s^{2} \theta_{1}$. If the motor is clamped, the transfer function between joint torque and motor torque is $\frac{\tau_{1}}{\tau_{m}}=R\left(r+\frac{J_{m}}{r k} s^{2}\right)^{-1}$. The transfer function between joint position and joint torque is: 
$\frac{\tau_{1}}{\theta_{1}}=\frac{-\frac{R^{2}}{r} J_{m} s^{2}}{r+\frac{J_{m}}{r k} s^{2}}=\frac{-k R^{2} J_{m} s^{2}}{k r^{2}+s^{2} J_{m}}$. Adding a PID feedback term, $\frac{\tau_{1}}{\theta_{1}}=\frac{-R^{2} J_{m} s^{2}}{r^{2}+\frac{J_{m}}{k} s^{2}+\left(k_{p}+k_{d} s+k_{i} / s\right)}$.

The closed-loop step response corresponding to this transfer function is displayed below, with model parameters set using values from the computer aided design.

In the case of multiple links (but still not considering link inertia), we again write $\tau_{m}+\tau_{k}=J_{m} \theta_{m}$ for the motor, but the elastic torque at the motor is now $\tau_{k}=-\left[r_{1} k_{1}\left(r_{1} \theta_{m}-R_{1} \theta_{1}\right)+r_{2} k_{2}\left(r_{2} \theta_{m}-R_{2} \theta_{2}\right)+r_{3} k_{3}\left(r_{3} \theta_{m}-R_{3} \theta_{3}\right)\right]$. The elastic torques at joints three, two, and one, are: $\tau_{3}=R_{3} k_{3}\left(r_{3} \theta_{m}-R_{3} \theta_{3}\right), \tau_{2}=R_{2} k_{2}\left(r_{2} \theta_{m}-R_{2} \theta_{2}\right)+R_{3} k_{3}\left(r_{3} \theta_{m}-R_{3} \theta_{3}\right)$, and $\tau_{1}=R_{1} k_{1}\left(r_{1} \theta_{m}-R_{1} \theta_{1}\right)+R_{2} k_{2}\left(r_{2} \theta_{m}-R_{2} \theta_{2}\right)+R_{3} k_{3}\left(r_{3} \theta_{m}-R_{3} \theta_{3}\right)$. Solving this system for $\theta_{1}, \theta_{2}$, and $\theta_{3}$ gives: $\theta_{1}=\frac{\left(\tau_{2}-\tau_{1}+k_{1} r_{1} R_{1} \theta_{m}\right)}{k_{1} R_{1}^{2}}, \theta_{2}=\frac{\left(\tau_{3}-\tau_{2}+k_{2} r_{2} R_{2} \theta_{m}\right)}{k_{2} R_{2}^{2}}, \theta_{3}=\frac{\left(-\tau_{3}+k_{3} r_{3} R_{3} \theta_{m}\right)}{k_{3} R_{3}^{2}}$, from which it

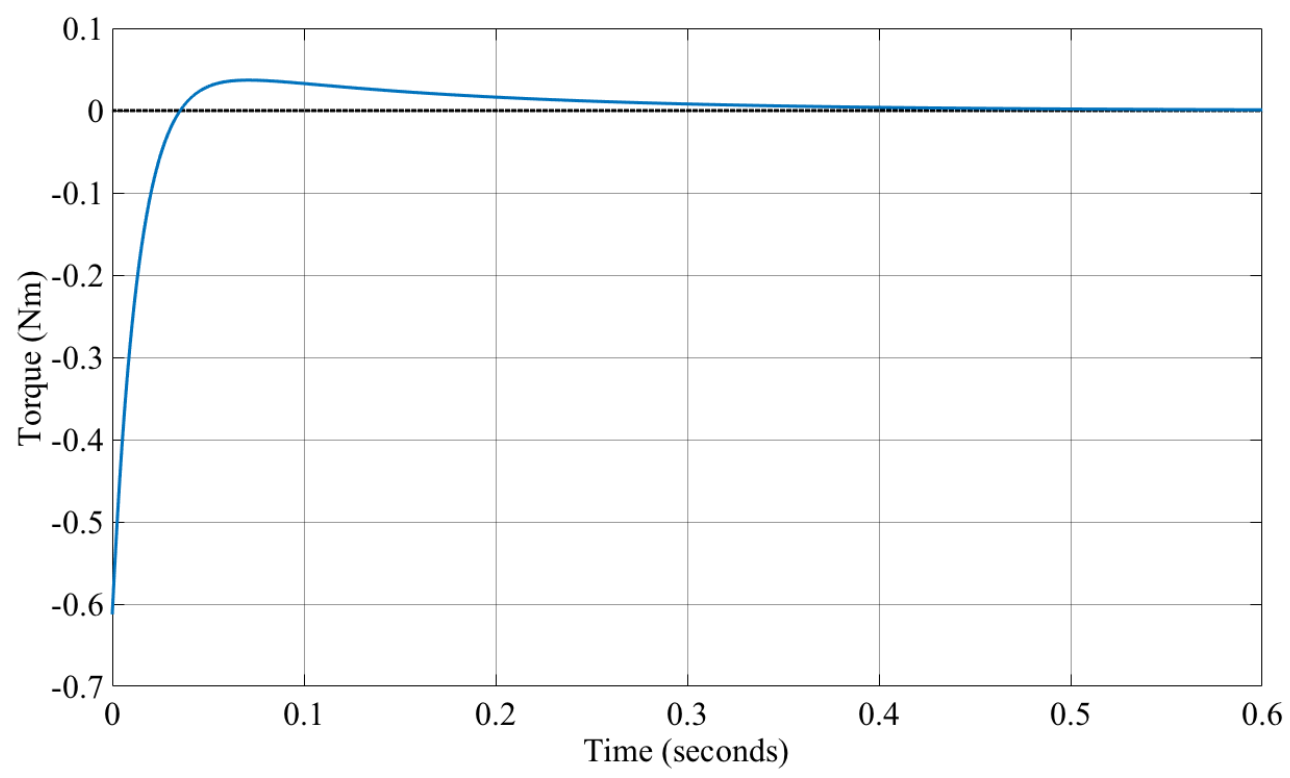

Fig. 7. Step response of the closed-loop, single-link SEA model. 
follows that $\tau_{k}=-\left[\frac{r_{1}}{R_{1}} \tau_{1}+\left(\frac{r_{2}}{R_{2}}-\frac{r_{1}}{R_{1}}\right) \tau_{2}+\left(\frac{r_{3}}{R_{3}}-\frac{r_{2}}{R_{2}}\right) \tau_{3}\right]$, and the transfer functions above can be altered accordingly.

\subsection{Electrical Design}

Each module is powered by a single external power supply, or internally by two 600 $\mathrm{mAh}, 3.7 \mathrm{~V}$ lithium polymer batteries per link. The batteries are wired in series along the module's central axis, and power the servomotor, rotary sensors, and an ARM Cortex M4 microcontroller, which operates as a microcontroller unit (MCU). Each pulley is actuated with an RX-28 servomotor, rated for a stall torque of $3.7 \mathrm{~N} \cdot \mathrm{m}$ at a no load speed of 79.4 RPM. Each servomotor features an inbuilt MAX485 driving chip and an angular encoder with $0.29^{\circ}$ resolution. Servomotors in adjacent modules are daisy-chained in sequence, so that linking two modules simply requires connecting a single power line. The robot receives high-level steering commands from a PC-based user interface, using $2.4 \mathrm{GHz}$ radio frequency transceiver modules. A small "head module," which draws power from the front-most bending module, carries the microcontroller and supporting electronics as well as a 9-axis inertial measurement unit (IMU), which acquires magnetic orientation, angular rates, and acceleration data at a $200 \mathrm{~Hz}$ update rate and transmits to the $\mathrm{MCU}$ via an $\mathrm{I}^{2} \mathrm{C}$ bus. Analog rotary position sensors located at each joint draw power locally from each module and relay joint angular displacement data to the MCU. Measurements when the robot is not encountering obstacles are used to calibrate the kinematic constraint constants in (1). A schematic of the full electrical design of the snake robot is displayed in Fig. 8. 


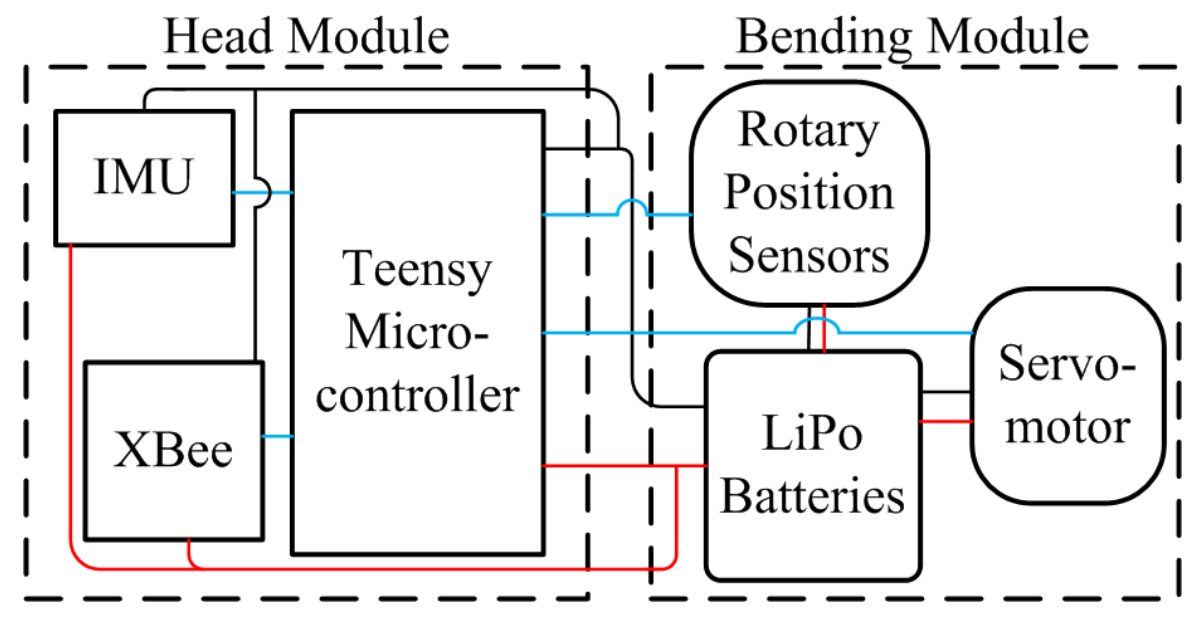

Fig. 8. Electrical layout of the full prototype.

The fully integrated prototype, pictured in Fig. 9, measures $53 \mathrm{~cm} \times 7 \mathrm{~cm} \times 7 \mathrm{~cm}$ at full extension, with a mass of $1.53 \mathrm{~kg}$, and permits joint angles of $55^{\circ}, 48.5^{\circ}$, and $42^{\circ}$ at joints one, two, and three in each module.

\subsection{Straight-Line Cable Displacements}

Straight cable routing schemes, though advantageous for their simplicity, pose potential problems for antagonistically routed systems due to potential nonlinearities in the sum of the displacements that occur in cable pairs. The present analysis investigates these nonlinearities and means of offsetting them, with reference to the diagram displayed in Fig. 10. Here, the angles $\alpha$ and $\beta$ are design parameters that define the angles 


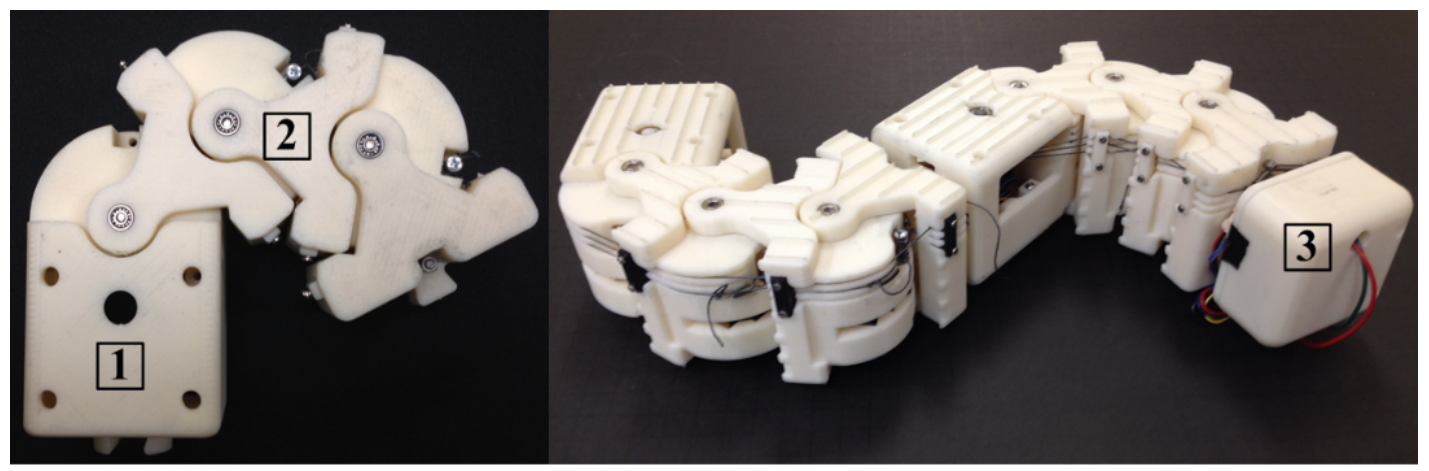

(a)

(b)

Fig. 9. (a) A single module, showing: (1) Actuation link, containing the servomotor. (2) A semicircular link. (b) Two-module snake robot prototype, showing (3) Head module, containing the MCU and IMU.

at which the cables connect to the lower portion of the $(i+1)$ th link and the upper portion of the $i$ th link, respectively, in terms of the local coordinate frames of the links. The lengths of the straight cable sections on the left and ride sides of the $i$ th link are given, respectively, by the norms

$$
L S_{i}=\left\|R_{i}\left(\left[\begin{array}{l}
0 \\
h_{i}
\end{array}\right]-R_{\alpha}\left[\begin{array}{l}
l_{i} \\
0
\end{array}\right]\right)+R_{i-1} R_{-\beta}\left[\begin{array}{l}
l_{i} \\
0
\end{array}\right]\right\|_{2}, R S_{i}=\left\|R_{i}\left(\left[\begin{array}{l}
0 \\
h_{i}
\end{array}\right]+R_{-\alpha}\left[\begin{array}{l}
l_{i} \\
0
\end{array}\right]\right)-R_{i-1} R_{\beta}\left[\begin{array}{c}
l_{i} \\
0
\end{array}\right]\right\|_{2}
$$

where $R_{i}$ is the rotation matrix corresponding to the angle $\theta_{i}$, and $R_{\alpha}$ and $R_{\beta}$ are the rotation matrices for the angles $\alpha$ and $\beta$, respectively.

The total change in the length of the $n$th cable (i.e. the cable that originates on the $n$th pulley section and terminates on the $n$th link) required to accommodate a pulley rotation of $\theta_{P}$ is given by the sum of the segment displacements on the two sides: 


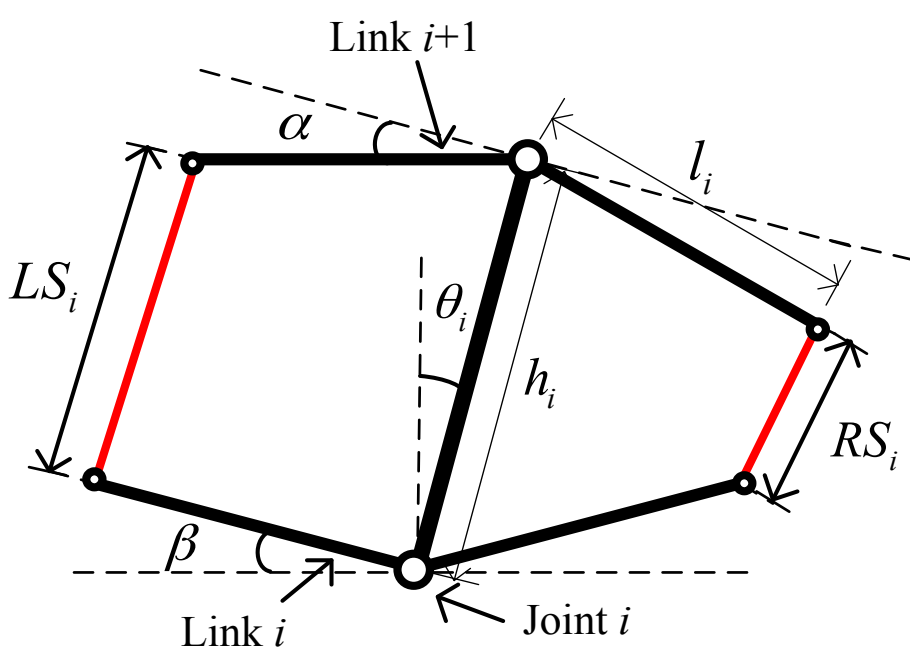

Fig. 10. Straight Wiring Scheme

$$
\begin{gathered}
\Delta_{n}=\Delta_{L, n}+\Delta_{R, n} \\
\Delta_{L, n}=\sum_{i=1}^{n}\left(L S_{i}-L S_{i, 0}\right)+r_{n} \theta_{P} \\
\Delta_{R, n}=\sum_{i=1}^{n}\left(R S_{i}-R S_{i, 0}\right)-r_{n} \theta_{P}
\end{gathered}
$$

where $L S_{i, 0}$ and $R S_{i, 0}$ are the values of $L S_{i}$ and $R S_{i}$ when the mechanism is straight (i.e. $\theta_{P}$ $=0)$.

Since cable length must remain constant, each $\Delta_{n}$ should equal zero for all $\theta_{P}$. However, as equation (4) shows, $\Delta_{n}$ in a mechanism described by (5) is nonlinear and, in general, nonzero for $\theta_{P} \neq 0$.

One method of compensating for cable nonlinearities is to place extension springs in series with the cables so that over-extended cables can shift their attachment point to accommodate desired angular displacements. Nonlinearities in cable displacements are thus replaced by nonlinearities in cable tensions, due to the forces exerted by the springs. Namely, a torque $\tau_{\text {Elastic }, i}=-k_{i} l_{i} \Delta_{i}$ is exerted on each joint, where $k_{i}$ is the spring constant of 
the $n$th extension spring. These nonlinearities can be approximately canceled out by adding a feedforward control torque. In order to compensate for the elastic torques occurring at each of the $N-1$ joints, the feedforward torque $\tau_{F F}$ must simultaneously satisfy the $N-1$ conditions:

$$
\tau_{\mathrm{FF}}=\sum_{i=1}^{N-1} r_{i}\left(\frac{k_{i} \Delta_{i} r_{i}}{R_{i}}-\frac{k_{i+1} \Delta_{i+1} r_{i+1}}{R_{i+1}}\right)
$$

Thus, if the ratios of the displacement errors in the cables remain approximately constant, spring constants may be selected according to (6) so that a single control term applied at the base motor approximately cancels the torques produced by the nonlinearities in cable displacement, at every joint.

Fig. 11(a) plots the total cable displacement in each of the three cables in a four-link mechanism described by (4), as it executes a series of sinusoidal bending motions. Here dimensional parameters were set to: $l_{i}=1.5 h_{i}$, with $l_{i}$ and $h_{i}$ constant for all $i, r_{1}=0.2 h_{i}, r_{2}$ $=1.5 r_{1}, r_{3}=2 r_{1}, \alpha=30^{\circ}$, and $\beta=10^{\circ}$, with an input frequency of $1.5 \mathrm{~Hz}$ and maximum joint angle of $35^{\circ}$. Fig. 11(b) plots the error $\Delta_{n}$ in the length of each cable as a percent of each cable's total length at the mechanism's home configuration. During this motion, $\Delta_{2} / \Delta_{1}$ and $\Delta_{3} / \Delta_{1}$ exhibit large nonlinearities as the mechanism approaches a singular configuration (i.e. as $\left\{\Delta_{1}, \Delta_{2}, \Delta_{3}\right\} \rightarrow 0$ ).

While (6) provides a means of compensating for cable displacement errors, it is also desirable to understand how these errors can be minimized or avoided by appropriate selection of model parameters. In particular, an understanding of the dependence of cable error on link geometry informs the process of design synthesis. For this purpose, a sensitivity analysis, displayed in Fig. 12, was performed on the percent error in cable 
displacement in a two-link mechanism with respect to $h / l$ as the mechanism bends, with the first joint angle $\theta_{1}$ in this case increasing from $0^{\circ}$ to $40^{\circ}$.
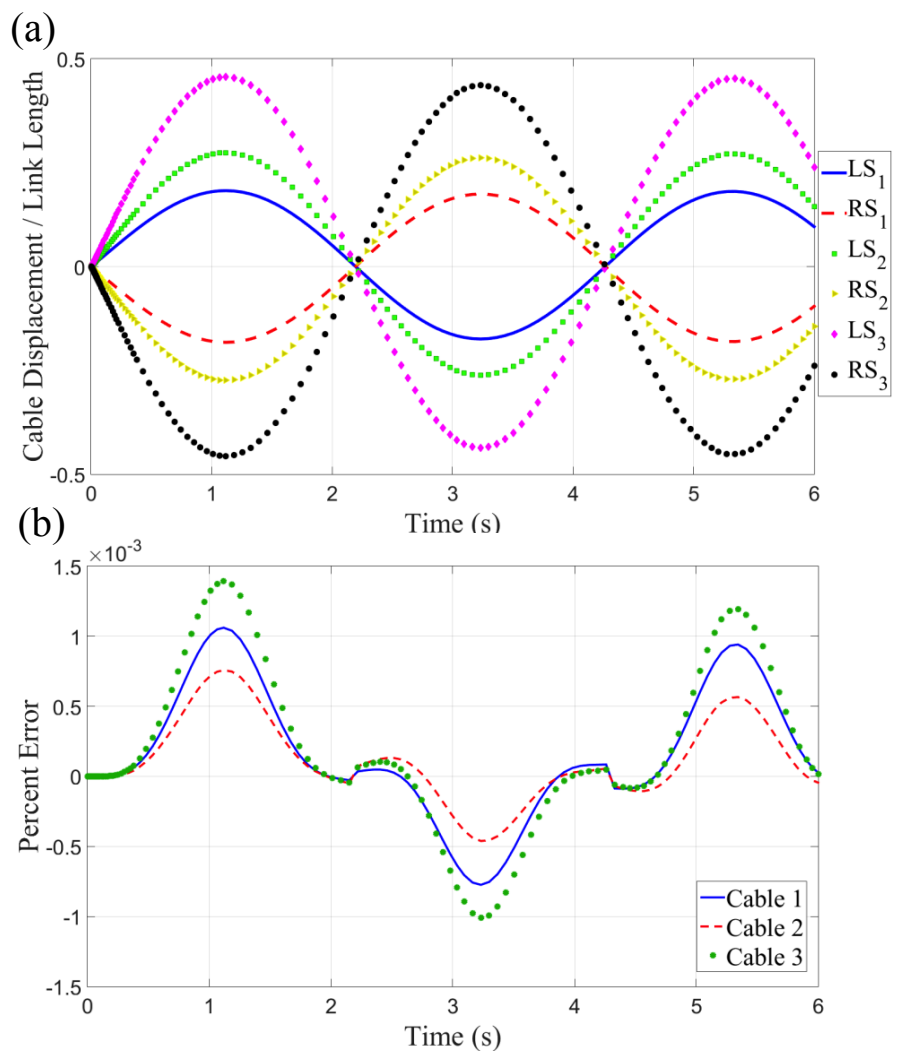

Fig. 11. Simulations of Cable Displacement (a) Cable Displacement / Link Length, (b) Percent Error

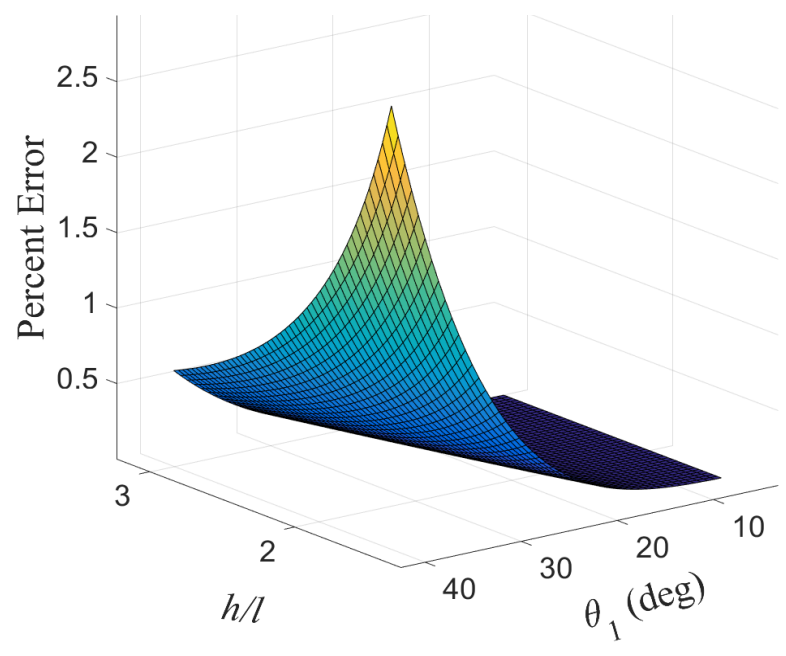

Fig. 12. Cable Displacement Error for Varying Link Geometry 
Here, the design parameters $\alpha$ and $\beta$ are taken to be zero. For large $\theta_{1}$, cable displacement error can be seen to increase sharply as $h / l$, the height to width ratio of the links, approaches zero.

\subsection{Circular Wiring Scheme}

While the nonlinearities that occur in straight cable routing schemes may be offset using the techniques described in Sec. 4.5, they may be avoided altogether by constraining the cables to follow circular paths. A schematic of the paths taken by cables around a bending mechanism with circular links is shown in Fig. 13. As in Sec. 4.5, the angle $\alpha$ specifies the last points at which the cables make contact with the lower portions of the links, in terms of the links' local coordinate frames. The points at which the cables contact the upper portion of each link's circular surface are defined for the left and right sides by the angles $\beta_{l}$ and $\beta_{r}$. The design parameter $\eta$ specifies the angle at which each link's circular arc extends beyond the local $x$-axis, where $\eta=0^{\circ}$ for a semicircle and $\eta=90^{\circ}$ for a full circle. Unlike in Sec. 4.5, $\alpha$ and $\beta$ are variable and may be determined by finding the line which is tangent to both circles for some $\theta_{i}$. In the case of equal, circular links, (constant $l_{i}$ ) where $\eta \geq 0^{\circ}$, the line between $\alpha$ and $\beta$ tracks $\theta_{i}$ and is therefore parallel to the line between circle centers. In this case, $\alpha=0^{\circ}$ and $\beta_{l}=-\beta_{r}$, so that for $\theta_{i} \leq \eta, L S_{i}=R S_{i}$ and the total cable length remains constant, that is, $\Delta_{n}=0$ for all $n \in\{1,2, \ldots, N-1\}$. Thus, the design parameter $\eta$ is equal to the maximum angular displacement that the links can undergo before encountering nonlinearities in cable displacement. 


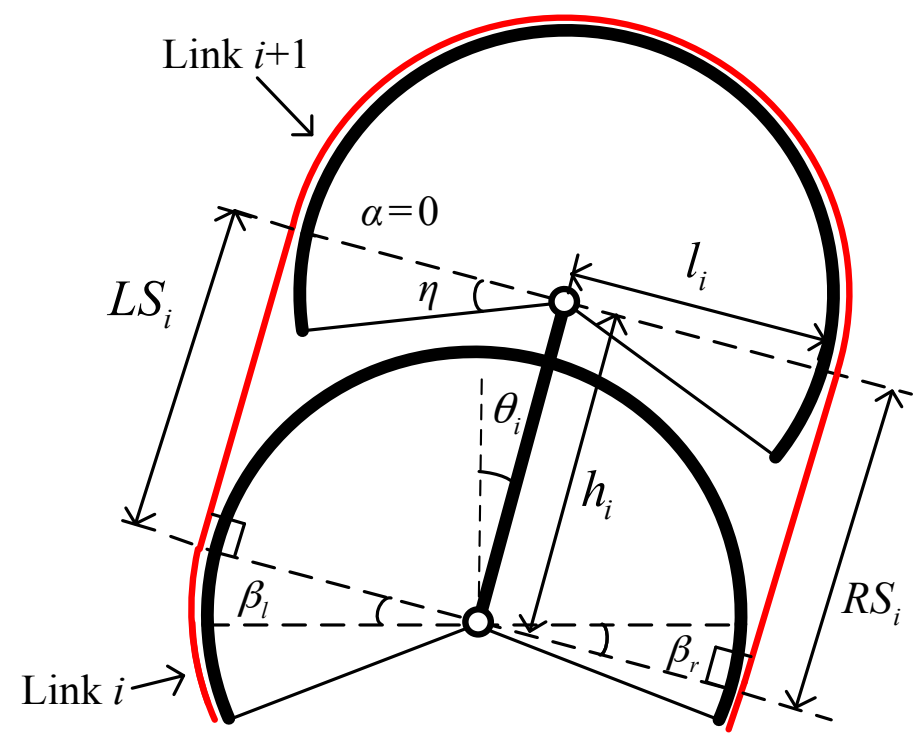

Fig. 13. Circular Wiring Scheme

In order to avoid mechanical interference between the circular and straight-line portions of adjacent links, link geometry must satisfy the condition

$$
\begin{cases}\eta \leq \frac{\pi}{2}-\arcsin \left(\frac{h}{2 l}\right) & \text { for } \frac{h}{l} \leq \sqrt{2} \\ \frac{h}{l}=\sin \eta+\sin \left(\frac{\pi}{2}+\eta\right) & \text { for } \frac{h}{l} \geq \sqrt{2}\end{cases}
$$

A design tradeoff must thus be made between minimizing the total length of the mechanism and maximizing the range of the joint angles over which $\Delta_{n}=0$. Nonlinearities that occur when $\theta_{i} \geq \eta$ may be dealt with by the same means as in Section 4.5 , since $\beta$ will remain fixed at the link's endpoint in this range.

In the Planar Bender, we have chosen to implement the circular wiring scheme described above using circular grooves, and we have chosen $\eta \approx 35^{\circ}$. 


\section{CHAPTER 5}

\section{DYNAMICS OF SERPENTINE LOCOMOTION}

\subsection{Lateral Undulation}

This section presents an overview of serpentine motion in snake robots that actuate every joint individually, and applies these principles to devise a locomotion strategy for a snake robot composed of underactuated bending mechanisms. A discrete approximation to the sinusoidal shape of a biological snake undergoing planar lateral undulation, for a snake robot with $N$ equal, straight links is given in terms of the snake's $i \in\{1, \ldots, N-1\}$ joint angles by the expression

$$
\theta_{\mathrm{ref}, i}=\alpha \sin (\omega t+(i-1) \delta)+\phi_{0}
$$

where $\alpha$ and $\omega$ are the amplitude and angular frequency of the angular displacement, respectively, $\delta$ is the phase offset between adjacent joints, and $\phi_{0}$ is a parameter which induces turning for nonzero values, assumed here to be constant [5]. Serpentine motion with constant $\alpha, \omega$, and $\phi_{0}$ (i.e. constant turning radius and speed) may thus be parameterized by a single variable, and is therefore a single degree-of-freedom motion, with an additional degree of freedom sufficient to parameterize planar turning.

\subsection{Derivation of an Approximation for the Optimal Joint Phase Offset}

Liljebäck et al. [5] showed that $\delta_{\text {opt, }}$, the $\delta$ that maximizes the speed of a snake robot (in a simplified dynamic model) during serpentine locomotion is given by the $\delta$ that maximizes

$$
k_{\delta}=\sum_{i=1}^{N-1} \sum_{j=1}^{N-1} c_{i j} \sin ((j-i) \delta)
$$


where $c_{i j}$ is the $i j$ th entry of $\mathbf{C}=\mathbf{A} \overline{\mathbf{D}} \in \mathbb{R}^{(N-1) \times(N-1)}$, with $\overline{\mathbf{D}}=\mathbf{D}^{\mathrm{T}}\left(\mathbf{D} \mathbf{D}^{\mathrm{T}}\right)^{-1} \in \mathbb{R}^{N \times(N-1)}$ and

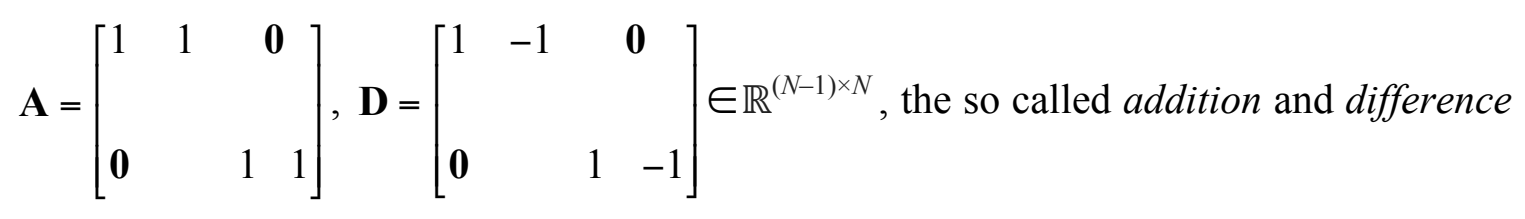
matrices.

We obtain a closed form approximation of $\delta$ as a function of $N$ by deriving some properties of $\mathbf{C}=\mathbf{A} \mathbf{D}^{\mathrm{T}}\left(\mathbf{D} \mathbf{D}^{\mathrm{T}}\right)^{-1}$. First of all, we define $n=N-1$, to clean up the notation.

We also define the matrix $\mathbf{B}=\left(\mathbf{D D}^{\mathrm{T}}\right)^{-1} \in \mathbb{R}^{n \times n}$, with $i j$ th element $b_{i j}$. Note that since a matrix times its transpose is symmetric, and the inverse of a symmetric matrix is also symmetric, B is symmetric. We will need the following:

Prop. 1. The sum of the $k$ th diagonal of $\mathbf{B} \in \mathbb{R}^{n \times n}$ is $b_{k} \equiv \frac{T_{k}}{n+1}$, where $T_{k}$ is the $k$ th tetrahedral number $T_{k}=\frac{1}{6} k(k+1)(k+2)$.

Note that by the $k$ th diagonal, we mean counting from the bottom left or top right corner of a matrix and moving toward the main diagonal. For example, a $\mathbf{B}$ matrix of dimension $n=4$, with each of the diagonals marked with its own bracket style, could be represented as:
$\mathbf{B}_{4}=\frac{1}{5}\left[\begin{array}{cccc}\langle 4\rangle & \{3\} & {[2]} & (1) \\ \{3\} & \langle 6\rangle & \{4\} & {[2]} \\ {[2]} & \{4\} & \langle 6\rangle & \{3\} \\ (1) & {[2]} & \{3\} & \langle 4\rangle\end{array}\right]$

To prove Prop. 1, we will need to show three things: 
1.1) The lower left element and the top right element of $\mathbf{B}$ are both $\frac{1}{(n+1)}$, and the rows and columns increases sequentially in units of $\frac{1}{(n+1)}$ as we move left along the top row, right along the bottom row, up along the leftmost column, or down along the rightmost column.

1.2) The first $n$ diagonals (starting at the bottom left corner) of a $\mathbf{B}$ matrix of dimension $n$ are precisely the diagonals of all of its lower dimensional counterparts, scaled by $\frac{1}{(n+1)}$

1.3) The sum of the main diagonal of $\mathbf{B}$ is $b_{n}=\frac{T_{n}}{n+1}$.

To prove 1.1., note that for the first column of $\mathbf{B},-b_{n-1,1}+2 b_{n, 1}=0 \Rightarrow b_{n-1,1}=2 b_{n, 1}$ and $-b_{n-2,1}+2 b_{n-1,1}-b_{n, 1}=0 \Rightarrow b_{n-1,1}=3 b_{n, 1}$. That the first column increases sequentially by factors of $b_{n, 1}$ from the $n$th element to the first can be proved by induction on the $i$ th element from the end, with base case $i=2$ (We've already shown cases $i=2$ and $i=3$, and case $i=0$ is true by definition). Namely, suppose $b_{n-i, 1}=(i+1) b_{n, 1}$ for some $i \geq 2$. Then, $-b_{n-i-1,1}+2 b_{n-i, 1}-b_{n-i+1,1}=0 \Rightarrow b_{n-i-1,1}=2(i+1) b_{n, 1}-i b_{n, 1}=(i+2) b_{n, 1} \Rightarrow b_{n-i-1,1}-b_{n-i, 1}=b_{n, 1}$, which proves the claim. Reaching the first element, we have $2 b_{1,1}-b_{2,1}=1 \Rightarrow 2 n b_{n, 1}-(n-1) b_{n, 1}=1 \Rightarrow b_{n, 1}=\frac{1}{(n+1)}$. Applying the same argument to the last column of $\mathbf{B}$ shows that its elements increase in units of $\frac{1}{(n+1)}$ from the top right corner to the lower right corner. Thus, since the matrix is symmetric, the top and bottom 
rows increase in the same way: right to left along the top row and left to right along the bottom row.

For 1.2., notice that the $i$ th element on the $k$ th diagonal, $(n-(k-1)+i, k+i)$ (where $k \in[1, n]$ and $i \in[1, k])$, is recursively generated by the two elements directly under it according to the rule $-b_{n-(k-1)+i, k+i}+2 b_{n-(k-1)+i+1, k+i}-b_{n-(k-1)+i+2, k+i}=0$ until we get to the second to bottom row, where $b_{n-(k-1)+i, k+i}=2 b_{n-(k-1)+i+1, k+i}$. Thus, the first $n$ diagonals of $\mathbf{B}$ are generated from the bottom row in the same manner for a $\mathbf{B}$ matrix of any dimension, and differ only by the bottom row's scaling factor of $\frac{1}{(n+1)}$.

Now for 1.3. Here $\mathbf{D D}^{\mathrm{T}}=\left[\begin{array}{cccc}2 & -1 & & \mathbf{0} \\ -1 & & & \\ & & & -1 \\ \mathbf{0} & & -1 & 2\end{array}\right]$ is the so called "second difference matrix," in which, by definition, the sum of the $k$ th lower diagonal, moving from the bottom left toward the main diagonal, is $2 \sum_{i=1}^{k} b_{i+(n-k), i}-\left(\sum_{i=1}^{k-1} b_{i+(n-k),(i-1)}+\sum_{i=1}^{k-1} b_{i+(n-k),(i+1)}\right)$. Since $\mathbf{D D}^{\mathrm{T}} \mathbf{B}=\mathbf{I} \in \mathbb{R}^{n \times n}, \operatorname{tr}\left(\mathbf{D D} \mathbf{D}^{\mathrm{T}} \mathbf{B}\right)=n$, and since $\left(\mathbf{D} \mathbf{D}^{\mathrm{T}}\right)^{-1}$ is symmetric, we can write $\sum_{i=1}^{n} b_{i, i}-\sum_{i=1}^{n-1} b_{i, i-1}=\frac{n}{2}$ for the main diagonal. So, the difference of the sums of the inmost two diagonals is related to the dimension of the matrix. Define $f(k)$ as the sum of the $k$ th diagonal in an $n$-dimensional $\mathbf{B}$ matrix, moving from the outside in. Then, for the case $k=n$, the difference of the sums of the inner two adjacent diagonals is given by the recursion relation $f(n)-f(n-1)=\frac{n}{2}=\frac{n}{2}(n+1) \frac{1}{n+1}$, which we recognize as $\frac{T_{n}}{n+1}$. 
We can now prove by inverse induction on $n$ that the $k$ th diagonal of a $\mathbf{B}$ matrix of dimension $n$ sums to $\frac{T_{k}}{n+1}$. This is clearly the case for $n=1$ or $n=2$. For $n=m \geq 2$, the sum of the main diagonal is $\frac{T_{m}}{m+1}$, and the sums of the outer diagonals are all given by $\frac{T_{k}}{m+1}$ by 1.3 , provided that the claim holds for all $n<m$, which is true by the induction hypothesis. This proves Prop. 1.

Now, multiplying $\left(\mathbf{D D}^{\mathrm{T}}\right)^{-1}$ from the left by the transpose difference matrix $\mathbf{D}^{\mathrm{T}}$ has the effect of subtracting adjacent diagonals, from the top right to the bottom left. In other words, the sum of the $k$ th diagonal $\sum_{i=1}^{n} b_{i+k, i}$ for some $k \in[0, n-1]$ becomes $\sum_{i=1}^{n} b_{i, j-k}-\sum_{i=1}^{n} b_{i+1, j-k}$. Thus, the sum of the $k$ th diagonal in the upper triangle of $\overline{\mathbf{D}}=\mathbf{D}^{\mathrm{T}}\left(\mathbf{D D}^{\mathrm{T}}\right)^{-1}$ becomes $\frac{T_{k}-T_{k-1}}{n+1}$ and the lower triangle is just the negative of this. But $T_{k}-T_{k-1}$ is just the $k$ th triangular number $t_{k}=\frac{1}{2} k(k+1)$. So to summarize, the sum of the $k$ th diagonal of a $\overline{\mathbf{D}}$ matrix of dimension $(n-1) \times n$ is $\bar{d}_{k} \equiv \pm \frac{t_{k}}{n+1}$, where the plus sign corresponds to the diagonals in $\overline{\mathbf{D}}$ 's upper triangle and the minus sign to those in the lower triangle.

We now show that the diagonals of $\mathbf{C}=\mathbf{A} \overline{\mathbf{D}}$ sum to $c_{k} \equiv \pm \frac{2 t_{k}}{n+1}$, except for the main diagonal, where the plus sign again corresponds to the diagonals in the upper triangle and the minus sign to those in the lower triangle. We will do this by looking separately at the 
upper and lower triangles of $\mathbf{N} \equiv \mathbf{A M}$, where $\mathbf{M}$ is an arbitrary matrix of dimension ( $n-$ 1) $\times n$. First note that left-side multiplication by the $\mathbf{A}$ matrix adds each row of $\mathbf{M}$ to the one above it, and the bottom row is cut off. But in the lower triangle of $\mathbf{M}$, moving an element up by one row also moves it up by one diagonal, so an alternate way of viewing leftmultiplication by $\mathbf{A}$ is that each diagonal is added to the one above it for $k \leq n-1$, and the element belonging to the first row of $\mathbf{M}$ is removed. But as we have already shown, the element of the $k$ th diagonal of the lower triangle of $\mathbf{M}$ that belongs to $\mathbf{M}$ 's bottom row is just $k$. So, denoting the sum of the $k$ th diagonal in the lower triangles of $\mathbf{N}$ and $\mathbf{M}$ as $n^{k}$ and $m_{k}$, respectively, $n_{k}=m_{k}+m_{k+1}-k$. But the recursion relation for the triangular sequence is just $f(k)=f(k-1)+k$, so $n_{k}=2 m_{k}$. The situation for the upper triangle is exactly the same, except that the element which is removed from the $k$ th diagonal of $\mathbf{M}$ is the one that belongs to M's top row, and as we have shown, the element of the $k$ th diagonal of the upper triangle of $\mathbf{M}$ that belongs to $\mathbf{M}$ 's top row is $k$. Thus, the sum of the $k$ th diagonal of $\mathbf{N}$ is twice the sum of the $k$ th diagonal of $\mathbf{M}$, except for the middle diagonal. We summarize these facts in the following result:

Prop. 2. The $k$ th diagonal of $\mathbf{C}$ sums to $c_{k}= \pm \frac{2 t_{k}}{n+1}$.

Given this fact about $\mathbf{C}$, we can now simplify the double sum $k_{\delta}=\sum_{i=1}^{n} \sum_{j=1}^{n} c_{i j} \sin ((j-i) \delta)$. Since the definition of the $k$ th diagonal is that $j-i=k$, for some $0<k<N-1$, the sine term is unchanged along each diagonal, and its sign flips exactly when C's sign flips. Thus, we can change $k_{\delta}$ to a sum over the diagonals: 


$$
\sum_{i=1}^{n} \sum_{j=1}^{n} c_{i j} \sin ((j-i) \delta)=2 \sum_{k=1}^{n} c_{k} \sin ((n-k) \delta)=2 \sum_{k=1}^{n} \frac{2 k(k+1) / 2}{n+1} \sin ((n-k) \delta)
$$

$=\left(\frac{2}{n+1}\right) \sum_{k=1}^{n} k(k+1) \sin ((n-k) \delta)$. Plugging in $N-1$ for $n$, we arrive at the following:

Prop. 3. $\quad k_{\delta}=\sum_{i=1}^{N-1} \sum_{j=1}^{N-1} c_{i j} \sin ((j-i) \delta)=\frac{2}{N} \sum_{k=1}^{N-1} k(k+1) \sin ((N-1-k) \delta)$

We can expand the summation:

$$
k_{\delta}=\frac{1}{4 N}\left[\csc ^{3}\left(\frac{\delta}{2}\right)\right]\left[(N-2)(N+1) \cos \left(\frac{1}{2} \delta\right)-N(N-1) \cos \left(\frac{3}{2} \delta\right)+2 \cos \left(\frac{1}{2} \delta(1-2 N)\right)\right]
$$

Taking a derivative with respect to $\delta$, and setting the result equal to zero,

$$
[1+N(N-1)] \cos \delta+(N-2) \cos (\delta N)-(N+1)[N-2+\cos (\delta(N-1))]=0
$$

Taylor expanding at $\delta=0$ and solving,

$$
\delta_{\mathrm{opt}} \approx \sqrt{\frac{30}{3 N^{2}-3 N+2}}
$$

Taking an additional term in the Taylor series,

$$
\delta_{\text {opt }} \approx \operatorname{Re} \sqrt{2} \sqrt{\frac{42 N^{2}-42 N+\sqrt{14}\left(-24 N^{4}+48 N^{3}-96 N^{2}+72 N-34\right)^{\frac{1}{2}}+28}{5 N^{4}-10 N^{3}+13 N^{2}-8 N+3}}
$$

This last expression approximates the exact solution to within 1.6 percent error for all $N \geq 3$, and falls below 0.1 percent error for all $N \geq 40$.

Note: It is easy to verify that $\delta=\frac{\pi}{N-1}$ maximizes $\sum_{k=1}^{N-1} k \sin ((N-1-k) \delta)$ and is also close to optimal for $k_{\delta}=\sum_{k=1}^{N-1} k(k+1) \sin ((N-1-k) \delta)$, so we can use this expression as a less unwieldy alternative to the Taylor expansion:

$$
\delta_{\text {opt }} \approx \frac{\pi}{N-1}
$$

This last expression provides us with a physical interpretation of $\delta_{\text {opt }}$ the optimal joint phase offset is approximately the value required for the first and last joint to be $\pi$ out of 
phase. In other words, a snake driving its joints at $\delta_{\text {opt }}$ attempts to form a discrete approximation to a single period of the serpenoid curve. Fig. 14 compares the three closedform approximations for $\delta_{\mathrm{opt}}$ in (11), (12), and (13) with numerical solutions of the two exact expressions in (9) and (10).

\subsection{Multibody Dynamic Model}

This section presents a multibody dynamic (MBD) model of a snake robot and a brief treatment of cable actuation in this context. The advantage of an MBD approach is that it removes the need for the idealized no side-slip constraints commonly employed in previous models, and clearly partitions forces internal to the mechanism into a vector of Lagrange multipliers.

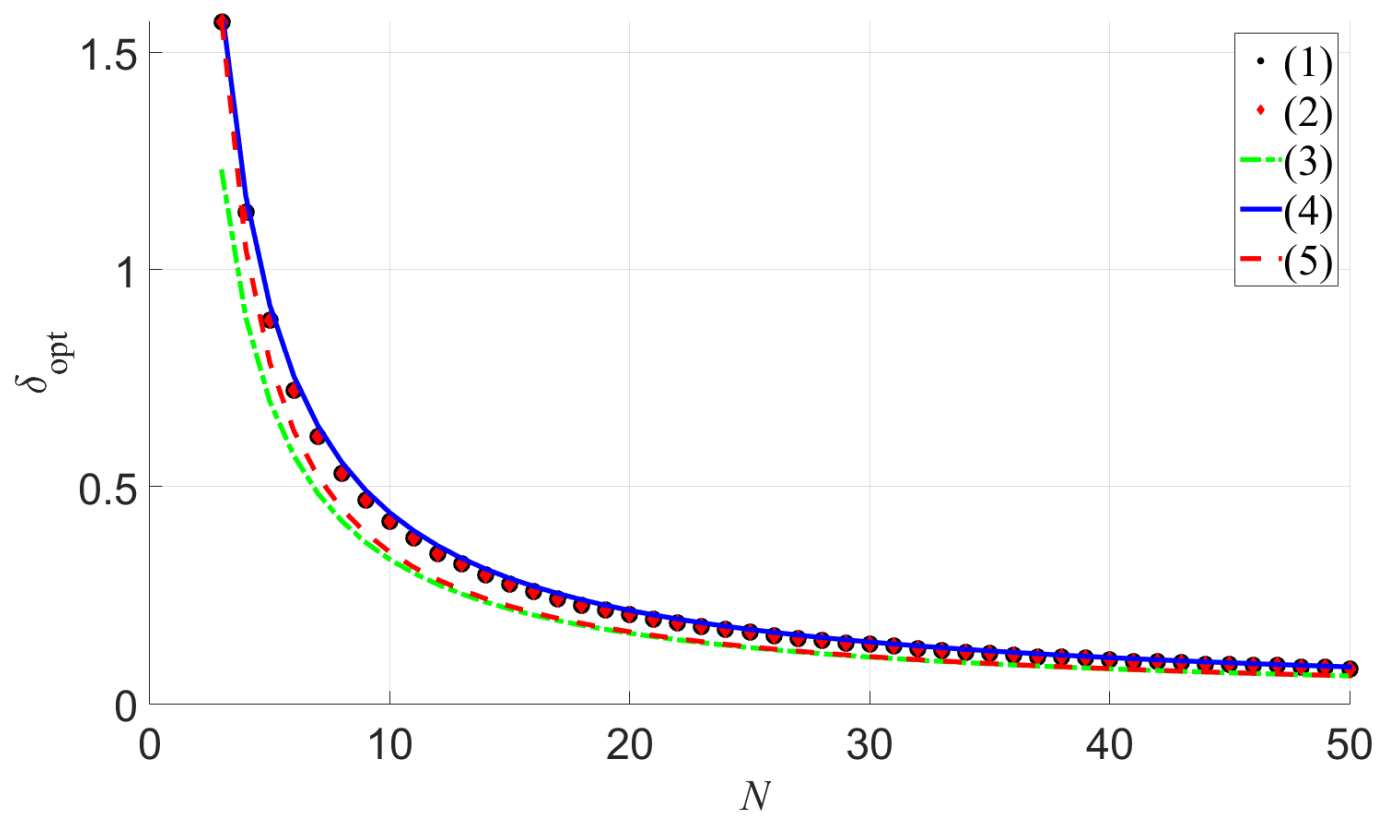

Fig. 14. Comparison of the three approximate expressions for $\delta_{\text {opt }}$, alongside the numerical solutions, for $N=3$ to 50 . 
The snake robot is modeled as $N$ straight, rigid links, connected in series by revolute joints, where link $i$ is of half-length $L_{i}$, mass $m_{i}$, and moment of inertia $I_{i}$. The positions of the $N$ link centers in the $x y$-plane are specified by a vector of generalized coordinates $\mathbf{q}=$ $\left[\mathbf{q}_{1}, \ldots, \mathbf{q}_{N}\right]^{\mathrm{T}}$ where $\mathbf{q}_{i}=\left[\mathbf{r}_{i}, \phi_{i}\right]^{\mathrm{T}}$ gives the global position $\mathbf{r}_{i}=\left[x_{i}, y_{i}\right]^{\mathrm{T}}$ and orientation $\phi_{i}$ of the $i$ th link, with respect to a fixed, global frame at the origin, as shown in Fig. 15. Each revolute joint is defined by two algebraic constraint equations, which are assembled into the vector equation $\boldsymbol{\Phi}(\mathbf{q}, t)=0$, of length $N_{c}=2(N-1)$.

The rotation matrix $\mathbf{R}_{i}$, corresponding to a rotation about the $z$-axis by an angle $\phi_{i}$, transforms the global position $\boldsymbol{r}_{i}$ of a link from the global frame to the $i$ th link frame. The local $x$ and $y$ coordinates are taken to be normal to the link and along the link, respectively.

frictional torque due to a pure rotation must be accounted for separately

The frictional force acting on the $i$ th $\mathrm{CM}$, in the global frame is:

$$
\begin{gathered}
\mathbf{F}_{f, i}=\mathbf{R}_{i} \mathbf{f}_{f, i} \\
\mathbf{f}_{f, i} \text { is the force in the local frame: } \\
\mathbf{f}_{f, i}=\left[f_{f, x, i} f_{f, y, i}\right]^{\mathrm{T}}=-\mathbf{\Omega}_{i} \mathbf{R}_{i}^{\mathrm{T}} \dot{\mathbf{r}}_{i}
\end{gathered}
$$

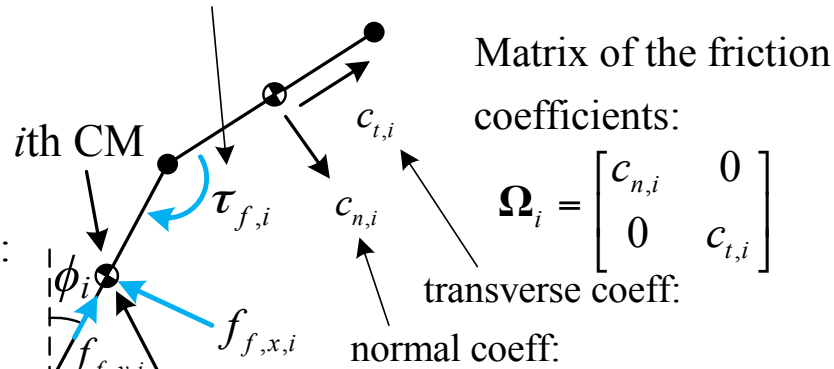

$\mathbf{R}_{i}$ is the rotation matrix for a rotation by $\phi_{i}$ about the global $z$-axis

$$
\mathbf{R}_{i}=\left[\begin{array}{cc}
\cos \phi_{i} & -\sin \phi_{i} \\
\sin \phi_{i} & \cos \phi_{i}
\end{array}\right]
$$
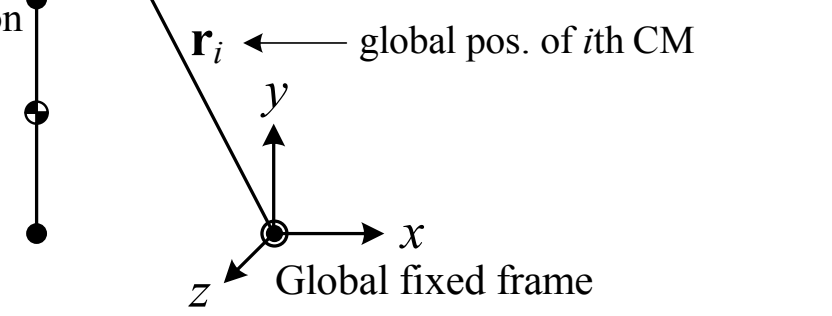

Fig. 15. Free body diagram of the snake robot, with coordinate and force definitions. 
The anisotropic frictional characteristics of the snake are modeled by defining normal and transverse coefficients of friction $c_{n}$ and $c_{t}$, which act along the local $x$ and $y$ directions, respectively. The external frictional force produced by the motion of the $i$ th link's center of mass (CM) directly depends on the global velocity vector $\mathbf{r}_{i}$. The friction force $\mathbf{F}_{f, i}\left(\mathbf{r}_{i}, \phi_{i}\right)$ acting on link $i$ is determined by rotating $\mathbf{r}_{i}$ into the local frame, to account for the locally defined frictional characteristics, and then rotating the local force back into the global frame. A viscous force on the CM of the $i$ th link is thus given by

$$
\mathbf{F}_{f, i}\left(\mathbf{r}_{i}, \phi_{i}\right)=-\mathbf{R}_{i} \mathbf{\Omega}_{i} \mathbf{R}_{i}^{\mathrm{T}} \mathbf{r}_{i}
$$

where $\boldsymbol{\Omega}_{i}=\operatorname{diag}\left(c_{n, i}, c_{t, i}\right)$ is a diagonal matrix of the normal and transverse coefficients of friction, respectively. Alternatively, a Coulomb frictional force acting on the $i$ th link's CM may be defined by

$$
\mathbf{F}_{f, i}\left(\mathbf{r}_{i}, \phi_{i}\right)=-m_{i} g \mathbf{R}_{i} \mathbf{\Omega}_{i} \mathbf{s g n}\left(\mathbf{R}_{i}^{\mathrm{T}} \mathbf{r}_{i}\right)
$$

where $g$ is gravitational acceleration and sgn is a vector operator which performs the signum function on each element of its argument. For simplicity, all simulations in this paper assume viscous friction.

The frictional forces due to translation of a link and pure rotation of the link about its CM must be accounted for separately. To account for the frictional torque produced by a pure rotation, each half-link is treated as consisting of $n$ equal masses, separated by a length $l_{n, i}=2 L_{i} /(2 n+1)$. For a link of orientation $\phi_{i}$ and angular velocity $\phi_{i}$, the $x$ and $y$ velocities of the $n$th segment due to a pure rotation of the link are $v_{x, n}=\phi_{i} l_{n, i} \cos \phi_{i}$ and $v_{y, n}=-\phi_{i} l_{n, i} \sin \phi_{i}$, respectively. The frictional forces on each segment may then be calculated using (14) or (15). Since the frictional forces on the $n$th segment are linear with 
$l_{n, i}$, the forces produced by the $n$ segments are equivalent to a force on a single mass, located at an effective length along the link of

$$
l_{\text {eff }, i}=l_{n, i} \sum_{n=0}^{n} \frac{n^{\prime}}{n}=\frac{n+1}{2 n+1}
$$

and in the limit that $n$ goes to infinity, $l_{\mathrm{eff}, i}=1 / 2$. Defining $\mathbf{v}_{i}=\phi_{i} l_{\mathrm{eff}, i}\left[\begin{array}{ll}\cos \phi_{i} & \sin \phi_{i}\end{array}\right]^{\mathrm{T}}$, the frictional torque acting on link $i$ may be written, with reference to (14) and (15), as

$$
\tau_{\mathrm{f}, i}=l_{\mathrm{eff}, i} \mathbf{F}_{\mathrm{f}, i}\left(\mathbf{v}_{i}, \phi_{i}\right)
$$

The torque produced at each of the joints by the action of the cables may be calculated by observing that all of the cable tensions acting on joint $i$ occur in equal and opposite pairs, except those acting between links $i-1$ and $i$. The relation between motor torque and joint torque is thus given in terms of the $i$ th link radius $R_{i}$ and cable tension $T_{i}$ by the system of equations

$$
\tau_{\mathrm{M}}=\sum_{i=1}^{N} F_{i} r_{i}, \tau_{i}=\sum_{j=i}^{N} F_{j} l_{j}
$$

where $\tau_{\mathrm{M}}$ is the torque applied by the motor and $r_{j}$ is the $j$ th pulley radius [21]. Springs of spring constant $k_{i}$ are attached in series with each of the cables, producing a cable tension on the $i$ th segment of $T_{i}=-k_{i} r_{i} \theta_{P}$, where $r_{i}$ is the $i$ th pulley section and $\theta_{P}$ is the pulley's angular displacement. The approximate angular displacement $\theta_{i}$ at each joint due to a pulley rotation of $\theta_{P}$ is given by the relation

$$
\theta_{i}=\alpha_{i} \theta_{\mathrm{P}}
$$

where $\alpha_{i}=\left(r_{i} / D_{i}-r_{i-1} / D_{i-1}\right)$ and $D_{i} \approx\left(L_{i}^{2}+R_{i}^{2}\right)^{1 / 2}$ [21]. The torque applied on joint $i$ by the springs is $\tau_{i}=-\kappa_{i} \theta_{i}$ where 


$$
\kappa_{i}=\alpha_{i} \sum_{i=j}^{N-1} \frac{k_{j} r_{j} R_{j}}{\alpha_{j}^{2}}
$$

Choosing the $i$ th spring constant according to (20) so that $\kappa_{i}=\kappa_{j}$ for all $i, j \in\{1, \ldots, N-1\}$, the effect of the linear springs may be treated as equivalent to that produced by equally valued torsional elements located at each joint. Positive spring constants obeying this condition may always be found, provided that $\alpha_{i}$ increases monotonically with increasing values of $i$.

The forces acting on each link are assembled into the generalized force vector $\mathbf{Q}^{\mathrm{A}}$. Defining a mass matrix $\mathbf{M}=\operatorname{diag}\left(m_{i}, m_{i}, I_{i}, \ldots, m_{N}, m_{N}, I_{N}\right)$, the $3 N+N_{c}$ constrained variational equations of motion may then be written as

$$
\delta \mathbf{q}^{\mathrm{T}}\left[\mathbf{M q}-\mathbf{Q}^{\mathrm{A}}\right]=0
$$

where $\delta \mathbf{q}$ is an infinitesimal displacement in the generalized coordinate vector $\mathbf{q}$, consistent with $\boldsymbol{\Phi}(\mathbf{q}, t)=0$ [22]. Introducing a vector $\lambda$ of Lagrange multipliers, the system may be reformulated as a matrix differential algebraic equation of motion (DAE)

$$
\left[\begin{array}{ccc}
\mathbf{M} & \boldsymbol{\Phi}_{\mathbf{q}}^{\mathrm{T}} & \mathbf{0} \\
\boldsymbol{\Phi}_{\mathbf{q}} & \mathbf{0} & \mathbf{0} \\
\mathbf{0} & \mathbf{0} & \mathbf{I}
\end{array}\right]\left[\begin{array}{c}
\mathbf{q} \\
\boldsymbol{\lambda} \\
\mathbf{q}
\end{array}\right]=\left[\begin{array}{c}
\mathbf{Q}^{\mathrm{A}} \\
\boldsymbol{\gamma} \\
\mathbf{q}
\end{array}\right]
$$

where $\boldsymbol{\Phi}_{\mathbf{q}}$ is the Jacobian and $\boldsymbol{\gamma}$ is a vector of acceleration independent terms [23]. Initial velocities must be consistent with the constraint $\boldsymbol{\Phi}_{\mathbf{q}} \mathbf{q}=-\boldsymbol{\Phi}_{t}$, where $\boldsymbol{\Phi}_{t}$ is the time derivative of the constraint vector $\boldsymbol{\Phi}$. Inverting the left-hand-side matrix in (22), the state vector $\mathbf{Y}=\left[\begin{array}{lll}\mathbf{q} & \boldsymbol{\lambda} & \mathbf{q}\end{array}\right]^{\mathrm{T}}$ may be found with the use of a numerical DAE solver. 


\subsection{Single Link Model of Lateral Undulation}

This section presents a semi-empirical model of serpentine locomotion that attempts to simplify the dynamics of lateral undulation by considering the forces acting on an individual link as it moves through a serpenoid-like trajectory. The goal of this approach is to predict, with less computational cost than a fully detailed dynamic model, the approximate value of the snake's steady-state speed, the size of the oscillations about this speed, and other characteristics of the motion, for arbitrary values of $\alpha$ and $\omega$. We compare this model to a detailed multibody dynamic (MBD) model, which we describe in [49], in which a snake robot drives its joints according to a PD control law: $\tau_{i}(t) \propto$ $k_{p}\left(\theta_{r e f, i}-\theta_{i}\right)+k_{d}\left(\dot{\theta}_{r e f, i}-\dot{\theta}_{i}\right)$, where $k_{p}$ and $k_{d}$ are gains.

We consider a snake robot with $N$ links of uniform length $L$ and mass $m$ undergoing lateral undulation in the $x y$-plane at constant period-averaged speed. Taking the snake's direction of travel to be along the $y$-axis and assuming viscous frictional contact, the acceleration of its $i$ th link $\mathbf{r}_{i}=\left[x_{i} y_{i}\right]^{\mathrm{T}}$ is given by

$$
\mathbf{r}_{i}=\frac{1}{m}\left[\begin{array}{cc}
-\left(c_{t} \cos ^{2} \phi_{i}+c_{n} \sin ^{2} \phi_{i}\right) & \left(c_{n}-c_{t}\right) \sin \phi_{i} \cos \phi_{i} \\
\left(c_{n}-c_{t}\right) \sin \phi_{i} \cos \phi_{i} & -\left(c_{t} \sin ^{2} \phi_{i}+c_{n} \cos ^{2} \phi_{i}\right)
\end{array}\right] \mathbf{r}_{i}
$$

where the terms on the main diagonal correspond to braking forces and those on the off diagonal correspond to propulsive forces [5]. Here, $\phi_{i}=a \sin (\omega t+(i-1) \delta)$ is the link's global orientation, of amplitude $a$, and $c_{n}$ and $c_{t}$ are the coefficients of friction in the normal and transverse directions of each link.

The maximum lateral displacement of a snake moving along the $y$-axis is half the snake's peak-to-peak distance, that is half the distance between links that are parallel to the $y$-axis. 
Suppose, without loss of generality, that the orientation of link $k$ at $t=0$ is set to $\phi_{k}=0$. The maximum lateral displacement is half the sum of the projections onto the $x$-axis of links $k$ through $m$, where $m$ is given by the constraint equation $\sum_{i=k}^{2 m-1+k} \phi_{i+1}=0$, which implies that $m=\pi / \delta$. Here, we have implicitly assumed that the snake contains an odd number of links, which we will take as a good approximation for the general case. The $x$-displacement of link $k$, provided that $a$ is small, is then: $x_{k}=-\frac{1}{2} \sum_{i=k}^{m} L \cos \left(\frac{\pi}{2}-\phi_{i}\right)=$ $-\frac{L}{2} \sum_{i=k}^{m} \sin [\operatorname{asin}(\omega t+i \delta)] \approx-\frac{L a}{2} \sum_{i=k}^{m} \sin (\omega t+i \delta)$. Expanding the summation,

$$
x_{k} \approx \frac{-L a}{2} \csc \left(\frac{\delta}{2}\right) \cos \left(\omega t+\frac{\delta}{2}\right)
$$

and $\dot{x}_{k} \approx \frac{L a \omega}{2} \csc \left(\frac{\delta}{2}\right) \sin \left(\omega t+\frac{\delta}{2}\right)$. But, since the snake is traveling at approximately constant speed and $\theta_{0} \approx 0$, each link must trace out the same approximate path over a period of oscillation [1]. By (23), the $y$-component of the snake's total period-averaged propulsive force, normalized by its body length, is then

$F_{\text {prop }} \approx\left(\frac{a \omega}{2 N}\right)\left(c_{n}-c_{t}\right) \csc \left(\frac{\delta}{2}\right)\left(\frac{\omega}{2 \pi}\right) \int_{0}^{2 \pi} \sin (\phi) \cos (\phi) \sin \left(\omega t+\frac{\delta}{2}\right) d t$

$\approx J 1(c n-c t) \frac{(a \omega) 2}{2 N} \csc \left(\frac{\delta}{2}\right) \cos \left(\frac{\delta}{2}\right)$, where $J_{n}(z)$ is the Bessel function of the first kind and where $\phi=a \sin (\omega t)$. Note that we require $\delta \approx \delta_{\text {opt }}$ since otherwise the assumption of the model that the snake approximately conforms to the serpenoid curve is not well obeyed. In the limit of large $N$,

$$
F_{\text {prop }} \approx \sqrt{10} J_{1}\left(c_{n}-c_{t}\right)(a \omega)^{2} \cos \left(\frac{\delta}{2}\right)
$$

since, by (11), $\lim _{N \rightarrow \infty} \delta_{o p t}=\frac{\sqrt{10}}{N}$. Thus, $\cos \left(\delta_{\text {opt }} / 2\right)$ is an approximate measure of the propulsive efficiency of a snake robot, growing monotonically as a function of the number of links the snake contains and reaching a maximum at $N=\infty$. The least efficient case, 
when $N=3$ and $\delta_{\text {opt }}=\pi / 2$, can be seen in this approximation to be $\sqrt{2}$ times less efficient than the limiting case of a continuous snake robot.

Under the conditions of straight, low-amplitude serpentine locomotion, the approximate, period-averaged dynamics of the snake's center of mass $(\mathrm{CM})$ are fully defined by a system of two differential equations from (23), together with the constraint in (24). At constantspeed, the CM acceleration in the direction of travel $\ddot{y}$ can be decoupled from $x$ and $\phi$ if the link angle amplitude $a$ is known. The amplitude $a$ reaches a maximum value $\bar{a}$ at steadystate speed, which can be approximated by a fit to the MBD model. We take the angular acceleration of the $i$ th joint to be $\ddot{\theta}_{l}=\tau_{i}(t) / I \propto(\alpha \omega / I) \cos [\omega t+(i-1) \delta]$, where $I$ is the link moment of inertia. We thus fit an expression of the form $\bar{a} \approx \eta \alpha /(\beta+I \omega)$ to the results of the MBD model, where $\eta$ and $\beta$ are fitting parameters.

Substituting the above approximations into (23) and making the change of variables $Y=$ $\dot{y}$, we may approximate the $y$-axis acceleration of the snake's CM as

$$
\begin{gathered}
Y(t)=a_{x}(t)-a_{y}(t) Y(t) \\
\left\{\begin{array}{l}
a_{x}(t)=\left(c_{n}-c_{t}\right) \frac{L \bar{a} \omega}{2 m} \csc \left(\frac{\delta}{2}\right) \sin \phi \cos \phi \sin \left(\omega t+\frac{\delta}{2}\right) \\
a_{y}(t)=\frac{1}{m}\left(c_{t} \sin ^{2} \phi+c_{n} \cos ^{2} \phi\right)
\end{array}\right.
\end{gathered}
$$

with $|\delta|<\pi$ required for locomotion. For straight-line, low-amplitude locomotion, the snake's $O(N)$ differential algebraic equations of motion (DAEs) can thus be approximated by a single first-order differential equation. Equation (26) was solved for $Y$ with the ode45 numerical solver in MATLAB over a 30 second interval, where the robot starts from rest and has model parameters $L=2, m=1, I=m L^{2} / 12, c_{n}=1, c_{t}=0, \gamma=\pi / 4, \eta=0.7$, and $\beta=$ 0.1 . The results of these simulation experiments are displayed for a 3-link snake robot in Fig. 16 for $\alpha=\{0.5,1\}$ rad and $\omega=\{2,5,10,20\} \mathrm{Hz}$. Because $a$ converges to $\bar{a}$, the single- 
link model will have a lower rise time than the detailed model, but should converge to the same steady-state speed. In Fig. 16, steady-state speed and the amplitude of oscillations about this speed can be seen to exhibit approximate qualitative and quantitative agreement across a wide range of $\alpha$ and $\omega$.

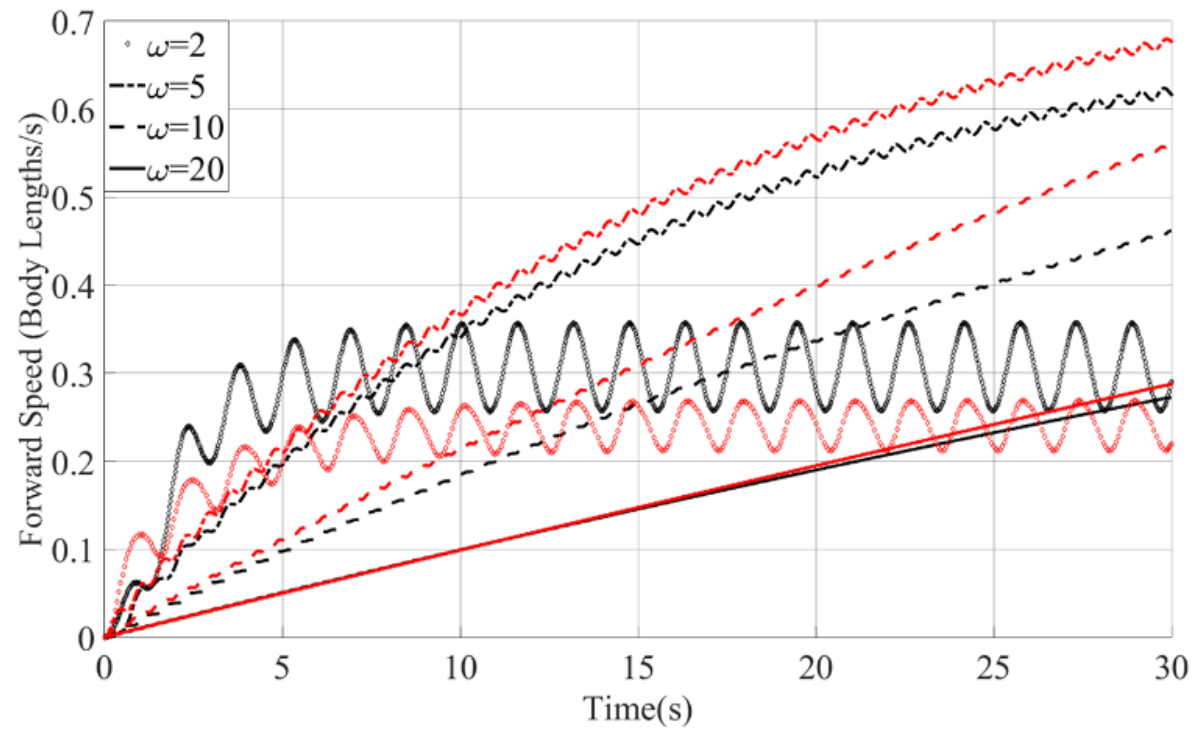

(a)

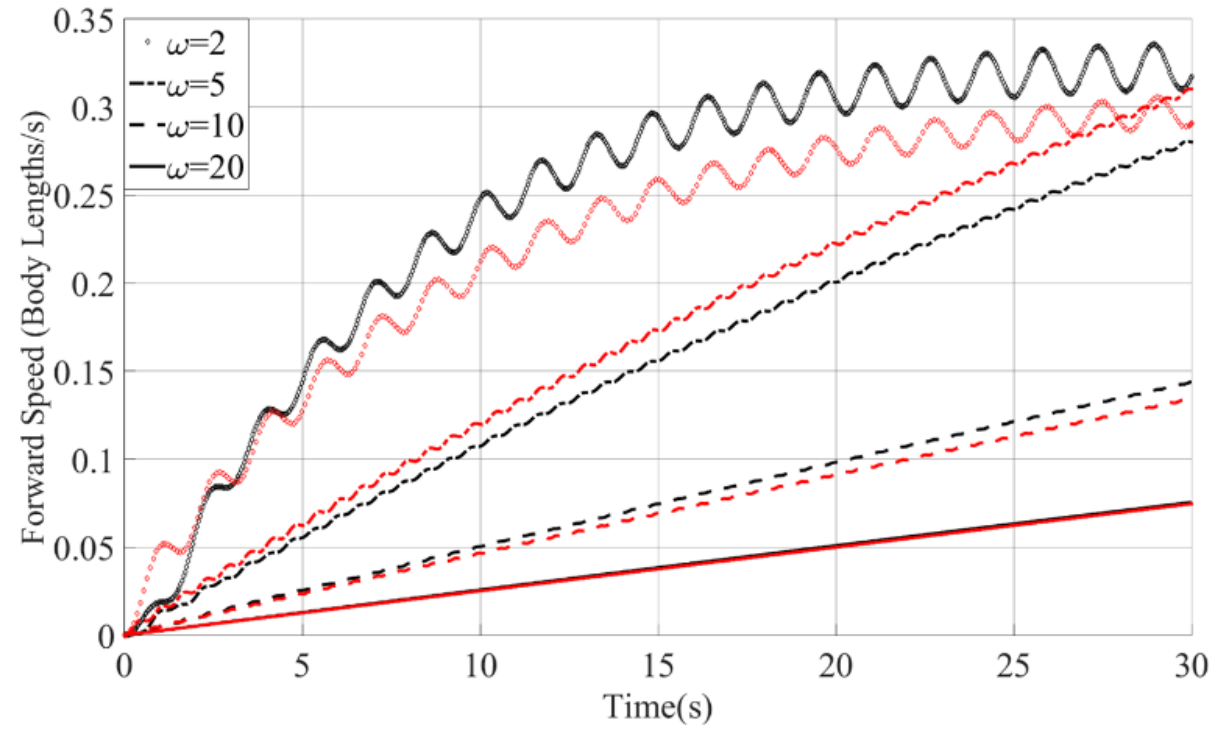

(b)

Fig. 16. Comparison of the full multibody dynamic model (black) with the singlelink model (light) at various oscillation frequencies for (a) $\alpha=1 \mathrm{rad}$ and (b) $\alpha=0.5 \mathrm{rad}$. 


\subsection{Bounds on Approximations by Discrete Linkages}

Imagine a half-period of a sine wave where the first and last points can slide up and down the $x$-axis as the amplitude of the wave changes, such that the arc length of the wave is invariant. It is obvious that such a curve can be perfectly approximated by a coupled linkage with infinite links, but less obvious that adding articulation improves the fit monotonically. We can show that increasing the articulation of a convex polygonal linkage by a factor of two always improves the fit to a half-period of a not necessarily static sine wave, by the following argument. Suppose that the wave is fit at some instant by a twolink linkage whose first and last points coincide with the first and last points of the sine wave, and which lies completely underneath it. At step $i$, draw two equal links for each link in step $i-1$ that end on the joints from step $i-1$. Also, require that each such link pair has a total length greater than the length of the link that they replace, but also that the sum of all link lengths at any step is less than the total arc length of the sine wave. By the triangle inequality, each such link pair will always lie between the link of the previous step and the sine wave.

We will now show bounds on how well $N$ uncoupled links can approximate a half period of a static sine wave, in terms of the difference in the areas enclosed by the two shapes $\varepsilon_{\text {Area }}$. To compute a lower bound, imagine a procedure in which at step $j$ we fit $j$ links of length $S_{j} / j$, where $S_{j}$ is the length of the full mechanism in that step and is no more than the arc length of the sine curve. In the $j$ th step, define $\delta_{i, j}$ as the distance from joint $i$ of the linkage to the sinusoid along the perpendicular bisector of joints $i-1$ and $i+1$. The area-error at this step is no greater than the area of the triangle formed by links $i$ and $i+1$ and the line joining joints $i-1$ and $i+1$, which is less than or equal to $\delta_{i, j} L$ (this only works 
because of the convexity of sine over a single period). So the area-error is $\varepsilon_{\text {Area }, j} \leq \sum_{i=1}^{j} \delta_{i, j} L_{j} \leq j \delta_{i, j, \text { max }}\left(S_{j} / j\right)=S_{j} \delta_{i, j, \text { max }}$ where $\delta_{i, j, \text { max }}$ is the maximum $\delta_{i, j}$ for this step. In the following step, we subdivide the triangular region into two sub-triangles formed by the intersection of the old perpendicular bisector with the sinusoid and the links from the previous step, with new links half the length of the previous step. This can be no better than the best case, since this is always one of the possible cases. In this step, $\delta_{i, j}{ }^{2}=(L / 2)^{2}-$ $\left(L^{2}-\delta_{i, j-1}^{2}\right) / 4$, so $\delta_{i, j}=\delta_{i, j-1} / 2$ and thus $\delta_{i, j} \propto 1 /\left(2^{j}+1\right)=1 /(N+1)$. Thus, $\varepsilon_{\text {Area }} \propto 1 / N$.

On the other hand, the area under a half-period of a sine wave can be approximated by evaluating sine at each of $N_{d}+1$ equally separated points on the $x$-axis and calculating the area $P_{A}$ of the resulting polygon:

$P_{A}=\frac{A \lambda}{4 N_{d}} \sum_{i=1}^{N_{d}}\left[2\left(N_{d}-i\right)+1\right]\left[\sin \left(\frac{i}{N_{d}} \frac{\pi}{2}\right)-\sin \left(\frac{i-1}{N_{d}} \frac{\pi}{2}\right)\right]=\frac{A \lambda}{4 N_{d}} \cot \left(\frac{\pi}{4 N_{d}}\right)$. We can insure that this approximation for the area under the sine curve is at least as good as the optimal linkage fit by ensuring that the projection onto the $x$-axis of the smallest interval $x_{\min }$ in the optimal fit is no less than $\lambda /\left(4 N_{d}\right)$. But $x_{\min } \geq L \cos \left(\theta_{\max }\right)=\lambda /(4 N) \cos [\arctan (2 \pi A / \lambda)]=$ $\lambda /(4 N)\left[(2 \pi A / \lambda)^{2}+1\right]^{-1 / 2}$, so we can set $N_{d}=N \sqrt{(2 \pi A / \lambda)^{2}+1}$. The area error from a fit by an $N$-link linkage is then $\Delta_{\text {Area }} \geq A \lambda / \pi-A \lambda \cot \left(\pi /\left(4 N_{d}\right)\right) /\left(4 N_{d}\right) \geq \pi A \lambda /\left(48 N_{d}^{2}\right) \propto 1 / N^{2}$. In summary, we've established that the area-error of the linkage is $O(1 / N)$ and $\Omega\left(1 / N^{2}\right)$.

\subsection{Online Estimates of Friction Coefficients}

Using the single link model to estimate $x_{i} / y_{i}$ at steady-state speed and using the fact that $\sum_{i=1}^{N} f_{x}\left(\phi_{i}\right) x_{i}$ and $\sum_{i=1}^{N} f_{y}\left(\phi_{i}\right) y_{i}$ are approximately in phase at steady-state, estimates of external frictional forces can be obtained using only the global speed and acceleration of 
the snake robot in the direction of travel. Assuming that the normal and transverse friction coefficients, $c_{n}$ and $c_{t}$, have some fixed ratio, (26) can then be used to make online estimates of the friction coefficients. (We make this assumption here for simplicity, but it can easily be removed by also measuring the speed and acceleration of the snake normal to the direction of travel.) In general, a snake robot's optimal control parameters are functions of $c_{n}$ and $c_{t}$, so estimates of these parameters can be used in e.g. a gain scheduling scheme on $\delta$, according to the relationship in [49]. Fig. 17 displays calculations of the normal friction coefficient $c_{n}$ in simulations of a 3-link snake robot, over a period of 60 seconds. We assume perfect knowledge of the forward speed and acceleration, and calculate the friction coefficients as estimates of the snake's speed in the normal direction are varied. The snake reaches $95 \%$ of its steady-state speed after about 27 seconds, at which point estimates of $c_{n}$ also reach approximately constant values. Whereas an exact solution using perfect knowledge of the velocities of each link accurately predicts the friction coefficients under all conditions, estimates using the single-link model require that the snake be near its steady-state speed.

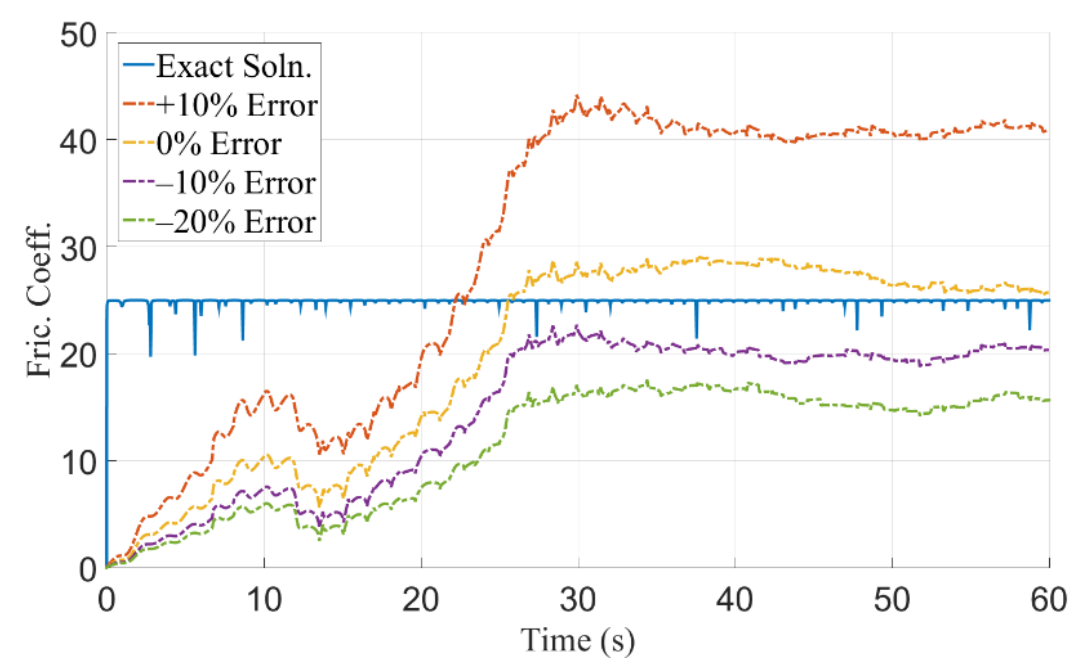

Fig. 17. Measurement of lateral friction coefficient, as errors are introduced into estimates of the snake's speed in the normal direction. 


\section{CHAPTER 6}

\section{CONTROL OF A KINEMATICALLY CONSTRAINED}

\section{SNAKE ROBOT}

We begin this section with a discussion of serpentine locomotion in a snake robot composed of kinematically coupled segments, such as the bending mechanism described in Sec. 4.1. We then introduce a controller that takes advantage of the increased articulation of the coupled units, and describe a method to perform a "best case" feedback linearization of the angular coordinates. Finally, we describe how the cable SEAs described in Sec. 4.2 can be used to perform force control and interact with obstacles during locomotion.

\subsection{Curvature Control}

As discussed in Sec. 5.1, a snake robot performing lateral undulation forms a discrete approximation to a sinusoid of invariant arc length, and the efficiency of the snake's locomotion depends on the closeness of this approximation. A group of kinematically coupled links such as the bending mechanism described in Sec. 4.1 can fit a section of a static sinusoid of fixed amplitude over wavelength $A / \lambda$ by an optimization of the coupling parameters, but can fit no more than a half period of this wave, since any more would require the mechanism to include an inflection point. Since the kinematic constraints in our mechanism are fixed, the closeness of the fit will decrease as $A / \lambda$ is varied, though no more than for a more highly articulated linkage. (It is easy to verify that, fitting a half-period of a sine wave from underneath with a convex polygonal linkage, it follows from the triangle inequality that the accuracy of the fit can always be improved by doubling the number of links in the linkage.) 
By analogy with (8), a snake robot composed of $i$ coupled linkages described by (2) can approximate the serpenoid curve by applying a control torque at the $i$ th motor of

$$
\tau_{\mathrm{M}, i}=k_{p}\left(\theta_{\mathrm{ref}, i}-\sum_{j=1}^{N_{b}-1} c_{j} \theta_{i, j}\right)+k_{d}\left(\theta_{\mathrm{ref}, i}-\sum_{j=1}^{N_{b}-1} c_{j} \theta_{i, j}\right)
$$

where $N_{b}$ is the number of links in each coupled linkage, $k_{P}$ and $k_{D}$ are proportional and derivative gains, $\theta_{i, j}$ is the $j$ th joint angle of the $i$ th linkage, $\theta_{\mathrm{ref}, i}$ is given by $(8)$, and $c_{j}=\left(r_{j}-\right.$ $\left.r_{j-1}\right) / r_{N-1}$ weights the joint angles by their contribution to bending, where $r_{j}$ is the $j$ th pulley radius. Here, the pulley radii were chosen by a least squares optimization of the link CMs to a sine wave of the desired $A / \lambda$ value.

The controller in (28) was demonstrated in a dynamic simulation of serpentine locomotion and turning in a two-bender snake robot. Each bending mechanism was represented as a serial chain of straight links connected by revolute joints and obeying the kinematic constraints specified by (1), with exterior frictional forces taken to be viscous. The sums of the joint angles in the two benders and corresponding motor torques are displayed in Fig. 18, for 70 seconds of snake locomotion. In the simulation in Fig. 18, the snake robot has a total mass of $1.4 \mathrm{~kg}$, pulley radii of $r_{1}=6 \mathrm{~cm}, r_{2}=11 \mathrm{~cm}$, and $r_{3}=16$ $\mathrm{cm}, l_{i}=35 \mathrm{~cm}$ for all links, and frictional coefficients of $c_{t}=0$ and $c_{l}=1$ in the transverse and lateral directions, respectively. The control parameters are set at $\alpha=1 \mathrm{rad}, \omega=2 \mathrm{~Hz}$, and $d=1.5 \mathrm{rad}$, with a maximum motor torque of $0.1 \mathrm{Nm}$. The snake is made to travel in a straight line between $t=0 \mathrm{~s}$ and $t=10 \mathrm{~s}$, turn $135^{\circ}$ clockwise between $t=10 \mathrm{~s}$ and $t=30$ $\mathrm{s}$, turn $90^{\circ}$ counterclockwise between $t=30 \mathrm{~s}$ and $t=50 \mathrm{~s}$, and then resume straight travel between $t=50 \mathrm{~s}$ and $t=70 \mathrm{~s}$. The intervals of turning may be identified in Fig. 18 as those periods of time during which the angle sums integrate to nonzero values. During its $70 \mathrm{~s}$ of travel, the snake robot moved a total distance of approximately $9.3 \mathrm{~m}$. The maximum 
steady velocity in simulation occurred at $d \approx 0.7 \mathrm{rad}$, about $45 \%$ lower than the prediction given by (9). This discrepancy is likely due to the presence of side slipping and details of link geometry.
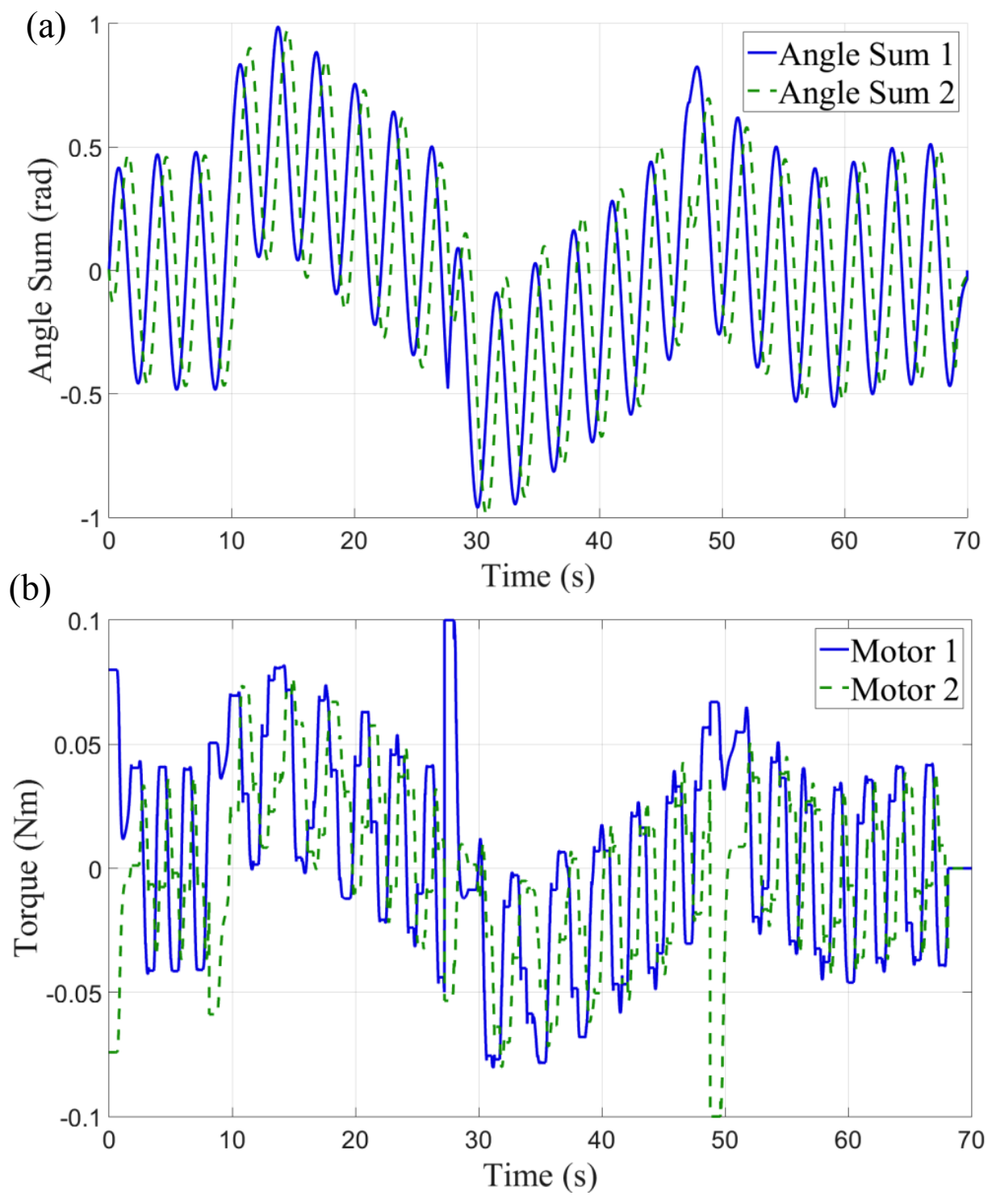

Fig. 18. Simulation of Snake Locomotion: (a) Sum of Joint Angular Displacements, (b) Motor Torques 
Trajectory and velocity results in a 120 s simulation are shown respectively in Fig. 19 and Fig. 20, where mass and length parameters are set using values from computer aided design, and with $A=5$ and $\omega=1$. The simulated snake robot can reach a maximum period averaged velocity on carpet of about 0.25 body lengths per second, after accelerating for about 7 seconds. The robot accelerates until it reaches the first waypoint at about 20 seconds, turns $45^{\circ}$ toward the second waypoint, which it reaches around 40 seconds, then sharply decelerates to execute a $90^{\circ}$ turn. Having lost velocity, it performs a low-radius-of-curvature turn in a series of jerks that rotate it without inducing significant forward motion, but then begins to accelerate again around 90 seconds and reaches the final waypoint at about 115 seconds.

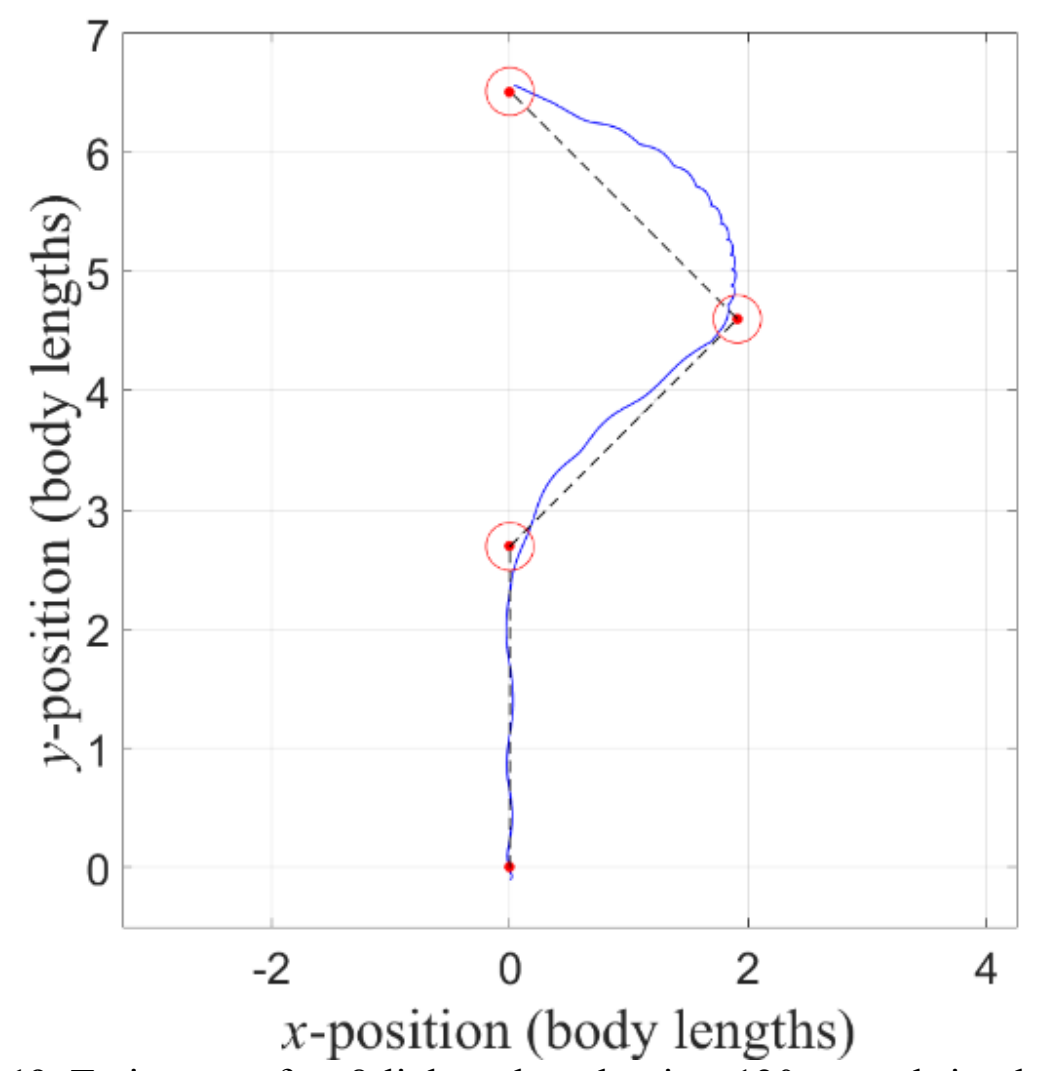

Fig. 19. Trajectory of an 8 -link snake robot in a 120 second simulation. 


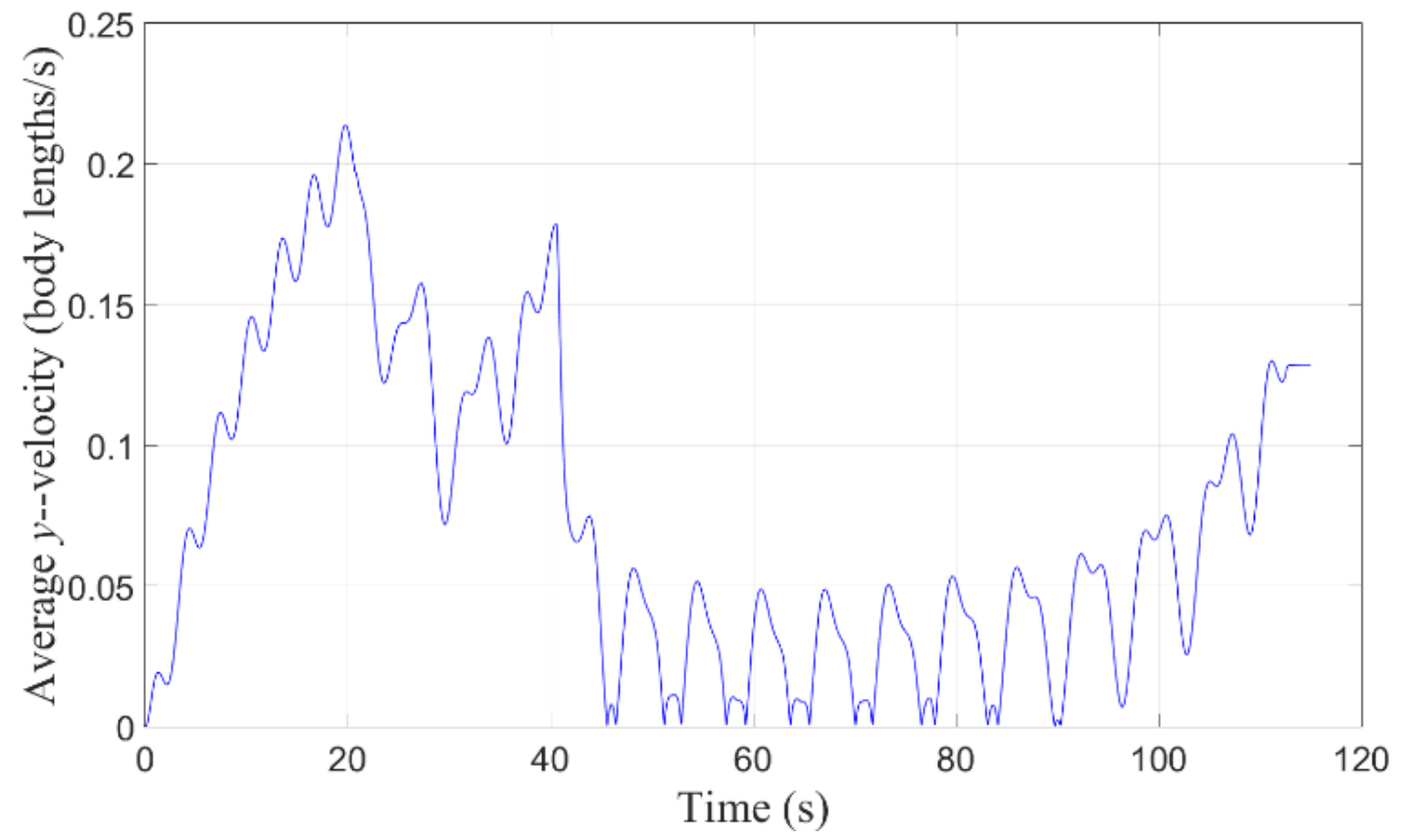

Fig. 20. Forward speed of an 8 -link snake robot in a 120 second simulation.

Using the MBD model of [49], we tested the effect of increased articulation in a twoDOF snake by comparing the simulated steady-state speed of a three-link snake with a snake composed of two, four-link coupled linkages. The two simulated snakes were of equal body length and both applied (28), with identical control parameters. The results of this simulation experiment, displayed in Fig. 21, indicate that the more highly articulated snake outperforms the three-link snake across all combinations of tested control parameters. 


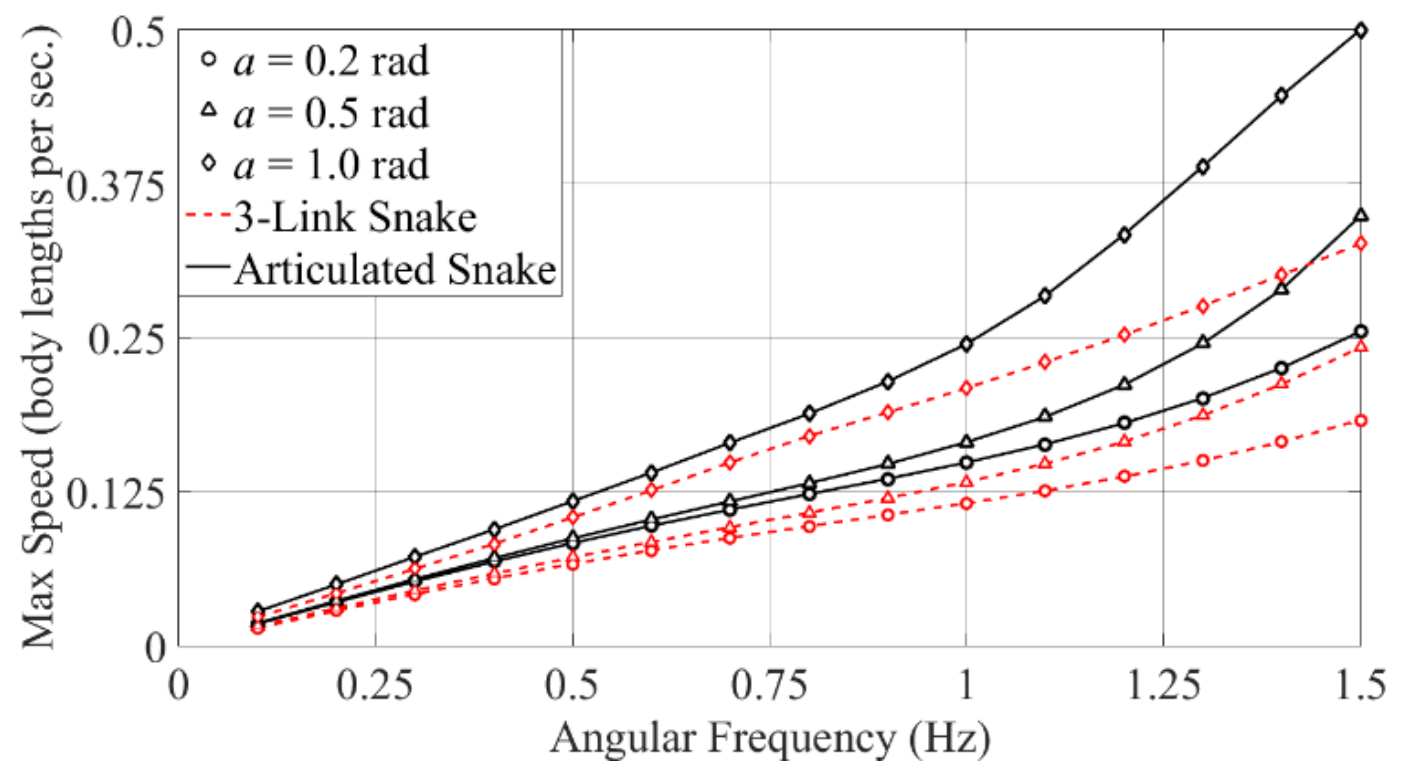

Fig. 21. Simulated steady-state speeds of two 2-DOF snake robots, one with three links (dotted line) and one with eight (solid line), as $a$ and $\omega$ are varied.

\subsection{Feedback Linearization}

As discussed in Sec. 5.3, an $N$-link snake robot moving in the plane can be modeled in a multibody dynamic approach as a serial chain of straight links joined by revolute joints, with $3 N$ DAEs per link and $2(N-1)$ per revolute constraint. The global position of the $i$ th link CM is specified by a vector of generalized coordinates $\mathbf{q}_{i}=\left[\mathbf{r}_{i}, \phi_{i}\right]^{\mathrm{T}}$, where $\mathbf{r}_{i}=\left[x_{i}, y_{i}\right]$ is the global position of the $i$ th link in the $x y$-plane and $\phi_{i}$ is its global orientation. Defining a vector of Lagrange multipliers $\lambda_{i}=\left[\lambda_{x, i}, \lambda_{y, i}\right]^{\mathrm{T}}$, a diagonal $3 \times 3$ generalized mass matrix $\mathbf{M}_{i}$, a generalized external force vector $\mathbf{F}_{\text {ext, }, i}$, and a vector of generalized actuation forces $\mathbf{U}_{i}$ for the $i$ th link, the system's equations of motion may be written in the form

$$
\left\{\begin{array}{c}
\mathbf{M}_{i} \mathbf{q}_{i}=\mathbf{F}_{\mathrm{ext}, i}+\mathbf{f}\left(\phi_{i}, \boldsymbol{\lambda}_{i}, \boldsymbol{\lambda}_{i-1}\right)+\mathbf{U}_{i} \\
\mathbf{r}_{j}-\mathbf{r}_{j+1}=\mathbf{g}\left(\phi_{j}, \phi_{j+1}, \phi_{j}, \phi_{j+1}\right)+\mathbf{h}\left(\phi_{j}, \phi_{j+1}, \phi_{j}, \phi_{j+1}\right)
\end{array}\right.
$$


( $i \in N$ and $j \in N-1$ ), where $\mathbf{f}, \mathbf{g}$, and $\mathbf{h}$ are vector valued functions that account for the mechanism's internal forces. Note that since joint torques are the only control inputs, only the angular components of $\mathbf{U}_{i}$ are nonzero: $\mathbf{U}_{i}=\left[0,0, U_{i}\right]^{\mathrm{T}}$.

Thus, as was observed in [50], the angular coordinates of a snake robot can be linearized by setting $U_{i}=U_{i \text {, lin }}+\bar{U}_{i}$, where $\bar{U}_{i}$ is set to track (4) and $U_{i, \text { lin }}$ is set to cancel undesirable forces, in this case the angular components of $\mathbf{F}_{\mathrm{ext}, i}$ and $\mathbf{f}$. Solving a system of $4 N-2$ equations for all of the $\lambda_{i}$, each of the $U_{i, \text { lin }}$ may be written as functions of the generalized coordinates and their derivatives.

The MBD model described above can be modified to represent a snake robot composed of the coupled linkages described in Sec. 4.2 by including the cable equations given by (2). The linearizing control input $U_{i, \text { lin }}$ at each joint can in this case be determined in exactly the same way as described above, but the system lacks sufficient DOFs to simultaneously apply these torques at each joint. We therefore take the following approach: the output $\tau_{\mathrm{M}}$ of the controller in (28) is input to a function $\mathbf{M} \rightarrow \mathbf{J}$, which determines the effect of a motor torque on each of the mechanism's joints. A feedback linearization is then performed exactly as before, outputting a linearizing control torque $U_{i}$ at each joint. Since by (2) the joint torques are all linear with the motor torque, the $U_{i}$ can then be input to a function $\mathbf{J} \rightarrow \mathbf{M}$, which solves the cable equations backwards to determine the torque that would be induced at the motor by each of the desired joint torques, and sums these values. The resulting "best case" linearized motor torque $\tau_{\mathrm{M}, \ell}$ is the motor torque that cancels the greatest possible fraction of the nonlinearities at each of the joints, since it is effectively an average over the linearizing joint torques. This method was tested in simulation in a snake robot composed of two 4-link bending modules, where estimates of the external forces 
were input to each of the $\mathbf{F}_{\text {ext, } i}$ and $\boldsymbol{\lambda}_{i}$. As shown in Fig. 22, feedback linearization increased the maximum achievable steady-state speed for all force estimates between $0 \%$ and $100 \%$ of the true values, with a maximum increase of between $15.5 \%$ and $27.5 \%$ for perfect force estimates.

\subsection{Path Following}

A snake robot driving its joint angles according to (8) moves in a straight line for $\theta_{0}=$ 0 and at constant radius of curvature for constant, nonzero $\theta_{0}$ [24]. In Liljebäck et al. [38], a path following controller is defined by setting

$$
\theta_{0}=A_{h}\left(h-h_{\text {ref }}\right)
$$

where the heading $h=\frac{1}{N-1} \sum_{i=1}^{N} \theta_{i}$ is the average joint angle, $A_{h}$ is a constant gain, and $h_{\text {ref }}$ is the desired heading, defined as

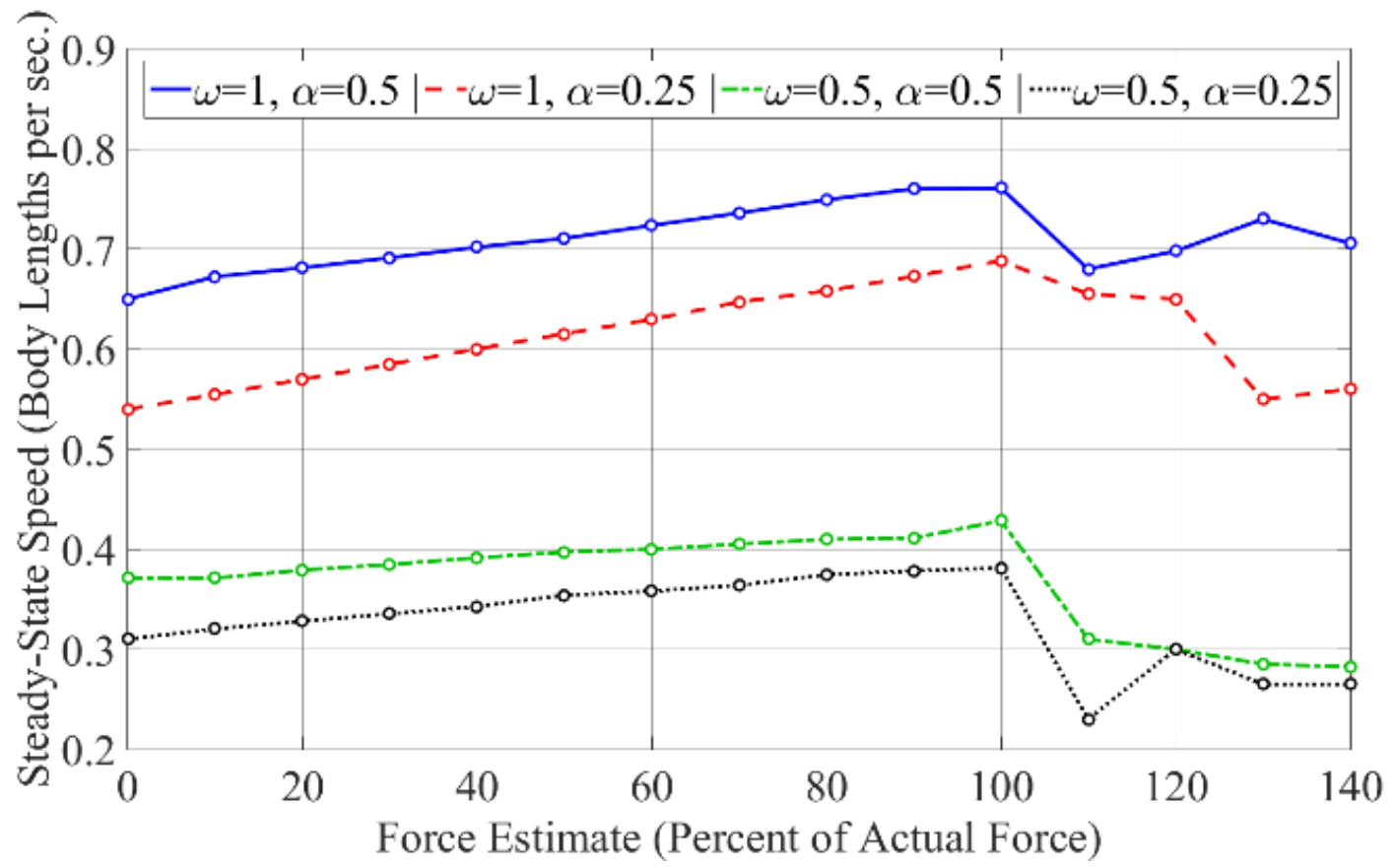

Fig. 22. Steady-state speed for a 2-module snake robot as estimates of external forces are varied between $0 \%$ and $140 \%$ percent of the actual values. 


$$
h_{\text {ref }}=-\arctan \left(P_{\mathrm{X}} / \Delta\right)
$$

where the "cross track error" $P_{\mathrm{X}}$ is the shortest distance from the snake's $\mathrm{CM}$ to the desired path and $\Delta$ is the constant "look-ahead distance."

Alternatively, the cross track error for the $i$ th link and $j$ th waypoint may be defined as

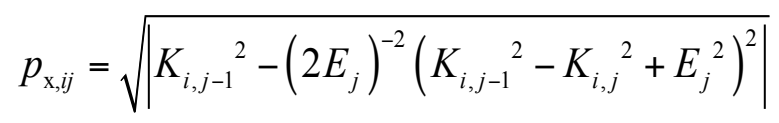

where $K_{i, j}$ is the distance from the $i$ th link frame to the $j$ th waypoint and $E_{j}$ is the distance between the (j-1)th and $j$ th waypoints. A single cross track error $p_{x, j}$ may then be defined as a weighted average over the links. Defining $\mathbf{v}_{i j}=\left[v_{i j, x} v_{i j, y}\right]^{\mathrm{T}}$ as the vector from the $i$ th link frame to the current waypoint and $\mathbf{w}_{j}=\left[\begin{array}{ll}w_{j, x} & w_{j, y}\end{array}\right]^{\mathrm{T}}$ as the vector between the current waypoint and the previous waypoint, a signed cross track error may be defined as $P_{\mathrm{X}, j}=-$ $\operatorname{sgn}\left(\theta_{\text {rel }}-\theta_{\mathrm{wp}}\right) p_{\mathrm{x}, j}$, where

$$
\theta_{\text {rel }}=\arctan \left(\frac{v_{i j, x}}{v_{i j, y}}\right), \quad \theta_{\text {wp }}=\arctan \left(\frac{w_{j, x}}{w_{j, y}}\right)
$$

Here, the signum function determines which side of the desired path the robot is on. The angle between desired paths $\theta_{\mathrm{wp}}$ is also used to ensure that when switching paths the robot always turns through the smaller angle between paths. In place of (31), the desired heading for the $j$ th waypoint is set according to

$$
h_{\mathrm{ref}}=-\arctan \left(P_{h} P_{\mathrm{X}, j}+I_{h} \int_{0}^{t} P_{\mathrm{X}, j} d t^{\prime}+D_{h} P_{\mathrm{X}, j}\right)+\theta_{\mathrm{wp}}
$$

The integral term in (34) acts to compensate for steady state error in the distance of the snake robot from the desired path, under large disturbance forces due, for example, to obstacles, water currents, or gravitational forces. Special care must be taken to avoid instabilities in the integral term under large errors, and in particular during the transient 
states when the snake is switching targets. Thus, if either the cross track error $P_{\mathrm{X}}$ or the heading error $\left|\theta_{\text {rel }}-\theta_{\mathrm{wp}}\right|$ pass some maximum values, the integral term is reset to zero. The heading gains must also obey the condition $I_{h} / P_{h}<H$ for a constant $H$, to prevent the snake from banking too hard. Fig. 23 displays a comparison of path-following errors with and without the integral term, for a 3-link, full-DOF snake following the line $x=0$, under a constant disturbance in the positive $x$-direction. The link length $L_{i}$ is taken to be a constant $L$ for all $i$, and the position of the robot's "head" (the end of its first link) is plotted in units of $L$.

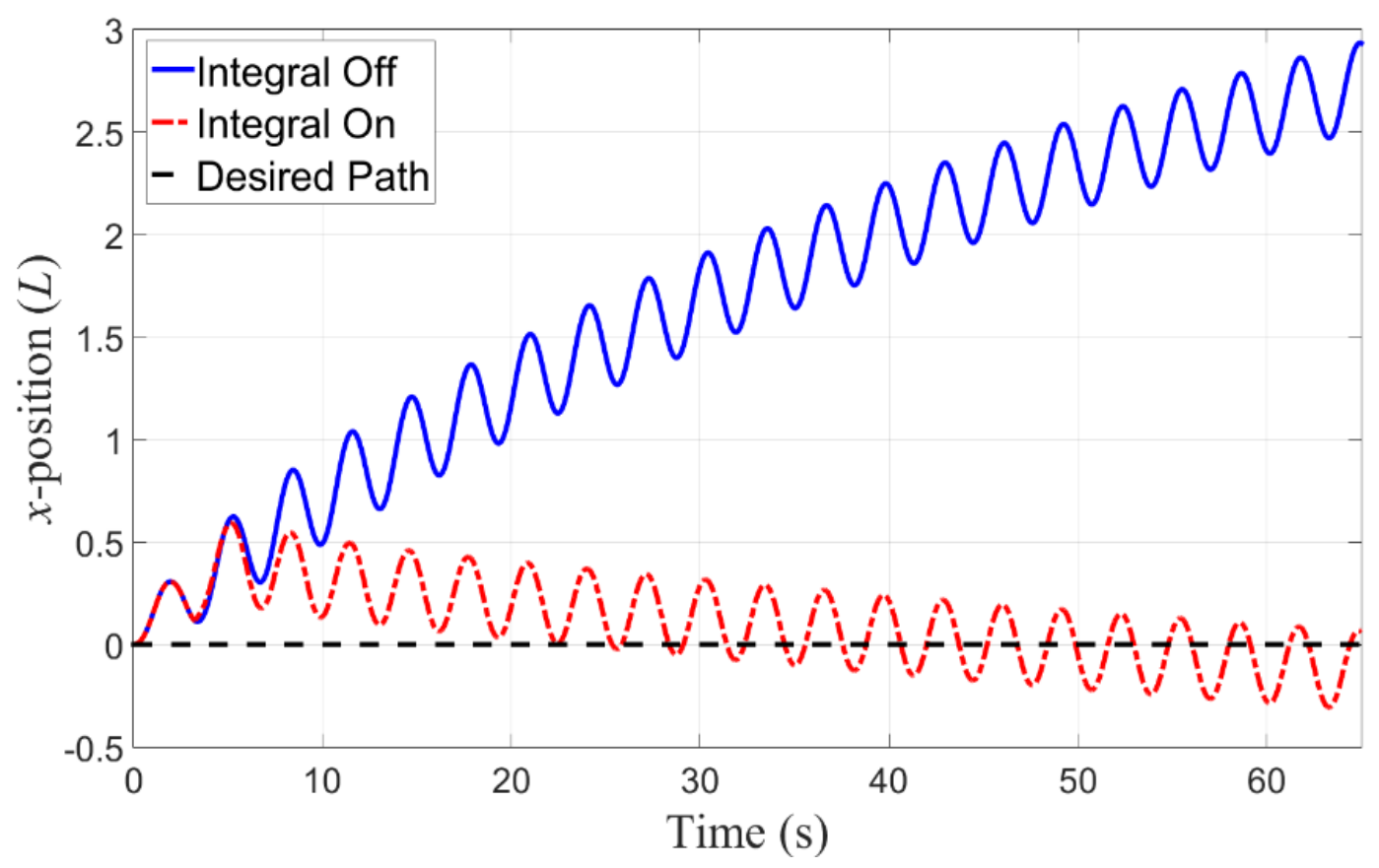

Fig. 23. Path following under a disturbance 
We can modify the controller further by setting $h_{r e f}=-\arctan \left[P_{h} P_{X, j}+\right.$ $\left.I_{h} \arctan \left(\int_{0}^{t} P_{X, j} d t^{\prime}\right)\right]+\phi_{p}$, so that, in the spirit of the full controller, the integral term avoids instability with the use of an arctangent function. Defining $\phi_{\text {AoA }}$ to run from $0^{\circ}$ to $180^{\circ}$ on one side of the path and from $0^{\circ}$ to $-180^{\circ}$ on the other, the controller can be improved by setting $P_{h}=p_{1}\left(\arctan ^{2}\left(p_{2} \phi_{\text {AoA }}\right)+p_{3}\right)$, where $p_{1}, p_{2}$, and $p_{3}$ are gains, so that the snake more aggressively approaches the desired path when the heading error is large. This definition of the heading gain ramps down the cross-track error correction as the snake approaches the correct heading in order to cause a smooth approach to the desired path. This approach was observed to increase the speed of a snake following a series of paths in simulation, essentially by decreasing path overshoot.

\subsection{Simulation Study 1: Phase Offset Optimization}

A reduced-DOF snake with $N_{\mathrm{b}}$ bending modules of $N$ links each has the same number of DOFs as a full-DOF snake with $\left(N_{\mathrm{b}}+1\right)$ links, but a structure similar to a full-DOF snake with $N_{\mathrm{b}} N$ links. We thus conjecture that the $\delta_{\text {opt }}$ that maximizes steady state speed in a reduced-DOF snake will fall between the optimal values for these two cases.

Fig. 24 displays $\delta_{\text {opt }}$ for full-DOF snake robots of various length, as $c_{n}$ in (15) is varied, and where $c_{t}=0$. The values of $\delta_{\text {opt }}$ for a reduced-DOF snake with equal link lengths, with $N_{\mathrm{b}}=2$ and $N=4$, are also displayed, and as expected, remain between the corresponding values for the 3 -link case and the 8 -link case. Unlike the prediction given by (9), $\delta_{\text {opt }}$ depends on $c_{n}$ in simulation. The results differ most sharply in the case of very low friction, where slipping is most pronounced. Note that $\delta_{\text {opt }}$ is undefined for $c_{n}=0$. 


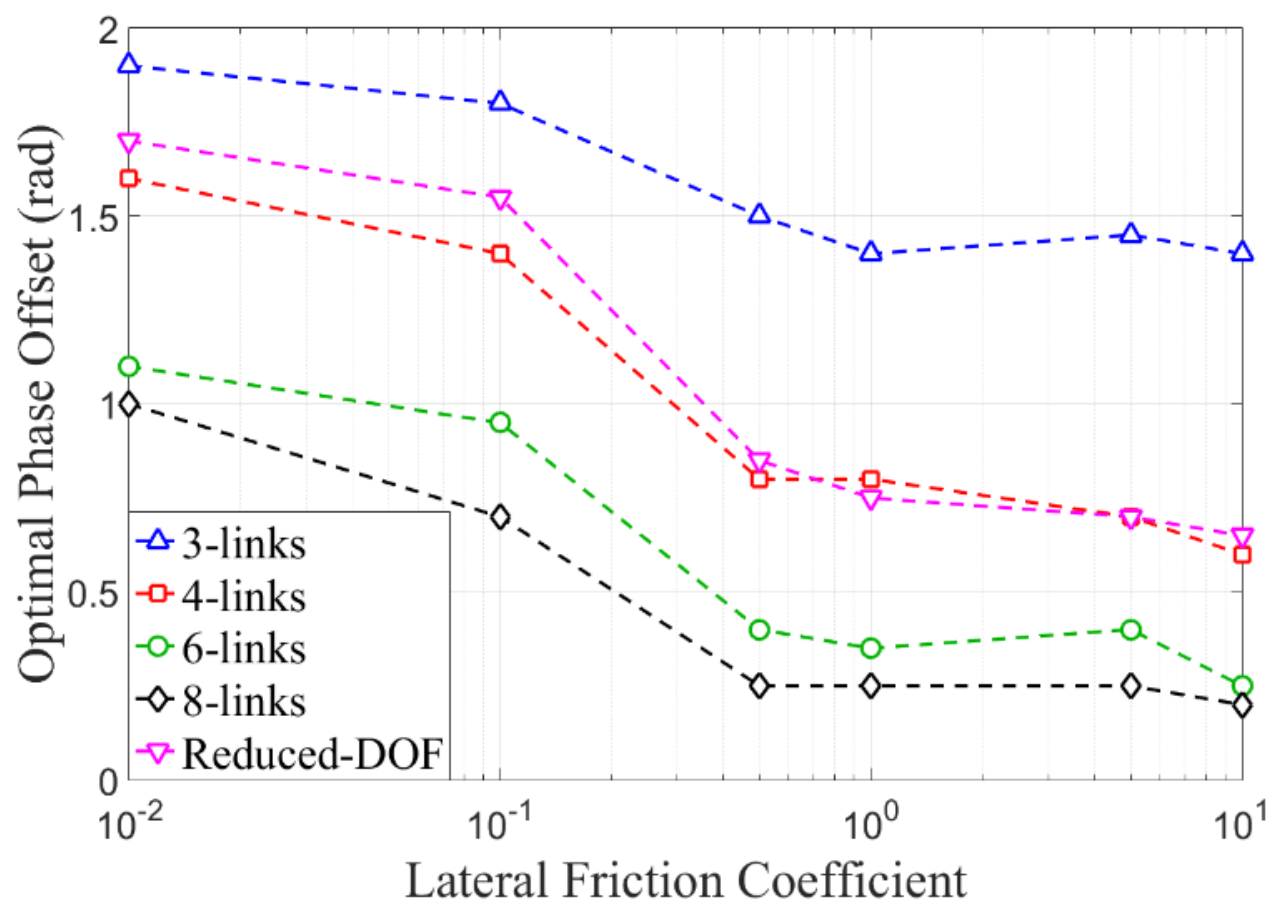

Fig. 24. Optimal phase-offset vs. lateral friction coefficient.

Fig. 25 displays steady state speed for a reduced-DOF snake robot with $N_{\mathrm{b}}=2, N=4$, and fixed $\delta$, as a function of bender geometry. Here, the middle two links are of constant length $L$ and the outer two links lengths $L_{\text {out }}$ are varied as some fraction of $L$, with mass and moment of inertia scaled according to link length. The steady state speed for each link length combination is a function of the ratio of the middle joint angle $\theta_{\text {mid }}$ to the outer two joint angles $\theta_{\text {side. }}$ As Fig. 25 shows, in the case of equal link lengths, steady state speed is maximized by setting $\theta_{\text {mid }}=\theta_{\text {side. }}$. The optimal joint angle ratio grows with increasing outer link length, so that the longer links remain parallel to the direction of motion for longer periods than the short links, and the drag force on the snake is minimized. 


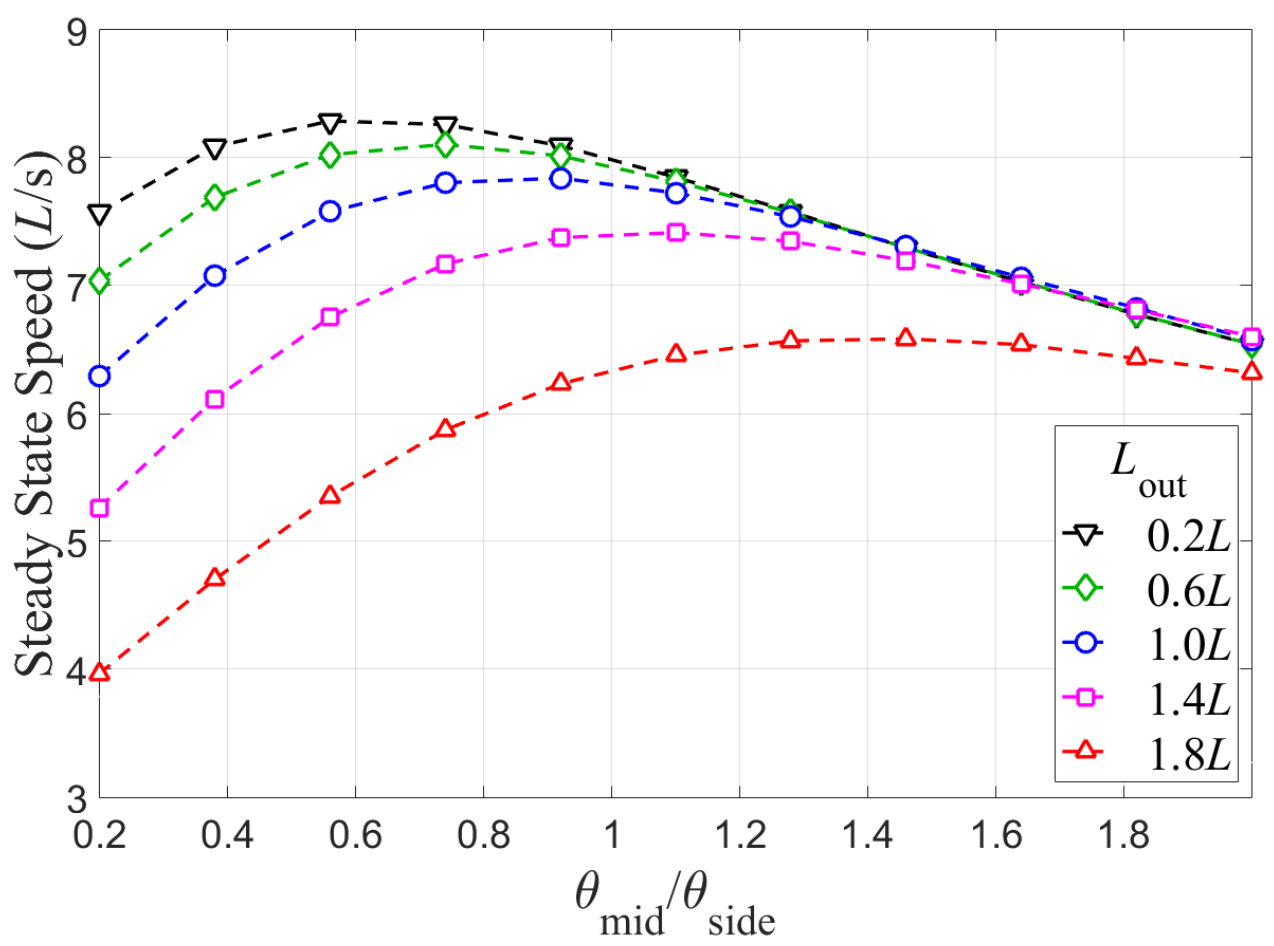

Fig. 25. Steady state speed vs. joint angle ratio.

\subsection{Force Control and Obstacle Interaction}

When the base of a bending module is fixed with respect to an external object, and under the assumption of static equilibrium, the torque acting on each joint is related to its angular displacement by a simple application of Hooke's Law. The joint torques $\boldsymbol{\tau}=\left[\tau_{i}, \ldots, \tau_{N-1}\right]^{\mathrm{T}}$ can then be written in terms of the net force $\mathbf{F}_{e}=\left[F_{e, x}, F_{e, y}\right]^{\mathrm{T}}$ applied by the end effector (the last link of the module) as $\boldsymbol{\tau}=\mathbf{J}^{\mathrm{T}}\left(\mathbf{R}_{0 e}\right)^{\mathrm{T}} \mathbf{F}_{e}$, where $\mathbf{J}$ is the module's Jacobian and $\mathbf{R}_{0 e}$ is the rotation matrix from the base frame to the end effector frame. Multiplying this equation by the pseudoinverse of $\mathbf{J}^{\mathrm{T}}$, the end effector force can be written as a function of the joint torques: $\mathbf{F}_{e}=\mathbf{R}_{0 e}\left(\mathbf{J} \mathbf{J}^{\mathrm{T}}\right)^{-1} \mathbf{J} \boldsymbol{\tau}$, where $\boldsymbol{\tau}$ is given in terms of the pulley angular displacement $\Delta \theta_{\mathrm{P}}$ by (1). Numerically solving the two resulting transcendental equations for the two components of $\mathbf{F}_{e}, \Delta \theta_{\mathrm{P}}$ can in general be written as a function of $F_{x}$ or $F_{y}$. Since the 
mechanism has only a single DOF, either component of $\mathbf{F}_{e}$, but not both, can be controlled by controlling $\Delta \theta_{\mathrm{P}}$, sufficient for pushing off of or grasping external objects.

Adaptation to obstacles can be handled by adding an obstacle offset $\theta_{\text {obs }}=$ $k_{o b s} \sum_{i=1}^{N_{b}-1} c_{i} e_{i}$ to $\theta_{0}$, where $k_{\mathrm{obs}}$ is a gain, $e_{i}$ is the obstacle induced displacement in the $i$ th joint, and $c_{i}$ is the joint weighting defined in Sec. 6.1. Here, we set $e_{i}$ as the $i$ th joint displacement measured by the rotary position sensor minus the angle predicted for this joint by the kinematic constraints in (1), for a pulley angle $\theta_{\mathrm{P}}$. This definition is essentially an application to an underactuated mechanism of the principle outlined in [51] and [52], where a snake robot is able to slide around obstacles by applying a control torque proportional to a power of obstacle-induced curvature. Alternately, we can accomplish the same effect without reference to pulley angle by comparing every joint to every other joint. There are $\left(\begin{array}{c}N-1 \\ 2\end{array}\right)$ combinations of joints, so we set $\theta_{\mathrm{obs}}=-k_{\mathrm{obs}} \varepsilon$, where

$$
\varepsilon=\frac{2}{(N-1)(N-2)} \sum_{j=1}^{N-1} \sum_{i=j+1}^{N-1}\left(\theta_{i}-C_{i j} \theta_{j}\right)
$$

and where $C_{i j}=\frac{r_{i}-r_{i-1}}{r_{j}-r_{j-1}}$ relates the $i$ th and $j$ th joints. Notice that, due to the SEAs, the application of a control torque of this form results in an equivalent spring force between the snake and an obstacle. The curvature controller and obstacle offset together constitute an impedance controller, which acts in parallel with the more local obstacle interaction behavior described in Sec. 4.3.

A block diagram of the full control algorithm for the snake robot is displayed in Fig. 26. In overview, the heading offset $\theta_{0}$ is set according to a path-following controller and modified by an obstacle offset, and is then input to the "curvature controller," which determines the desired motor torque $\boldsymbol{\tau}_{\mathrm{M}}$ in order to bend the module sinusoidally, taking 
as feedback the present positions of each of the joints. The resulting torque output is then modified by the partial feedback linearization block, which attempts to cancel the greatest possible portion of the nonlinear forces internal to the mechanism, and outputs a final, linearized motor torque $\tau_{\mathrm{M}, \ell}$ at the pulley. In the diagram, "Lin." represents the feedback linearization and "Force est." is an estimation of external frictional forces, consisting in this case of a viscous frictional model. In simulation, the force estimator was usually set to output a given percentage of the actual frictional forces, and the actual frictional forces were (optionally) overlaid with a Gaussian noise term.

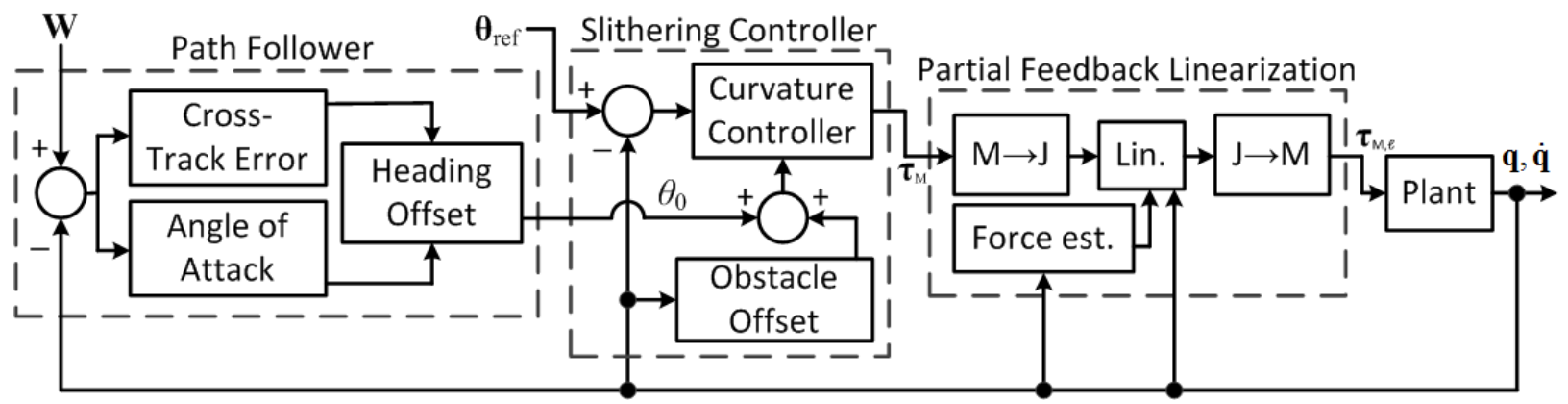

Fig. 26. The complete control scheme for the cable-driven snake robot. 


\section{CHAPTER 7}

\section{USE AS A MODULAR RECONFIGURABLE PLATFORM}

\subsection{Inchworm Locomotion}

In an $S$ cable routing scheme, where cables are alternately routed on the left and right sides of a link, the amount by which a segment rotates relative to the segment beneath it is given by a modification of (1): $\theta_{i}=(-1)^{i} \alpha_{i} \theta_{\mathrm{P}}$, with the same definition of $\alpha_{i}$, so ensuring that $r_{i} / D_{i}-r_{i-1} / D_{i-1}$ is constant for all $i$ guarantees that every other joint angle is equal and opposite, and the module will form an $S$-shape. A single module can thus approximate an entire period of a sinusoid. However, single-DOF slithering locomotion is not possible with this configuration, since there is no phase difference in the propagation of the sine wave along the length of the module, and the propulsive force averaged over an oscillation period is zero. However, $S$-routing can be used for single-module, inchworm like locomotion.

We can calculate the propulsive force contributed by the $i$ th link during the downward phase of its motion by solving the transcendental equation $L_{i-1} \sin \phi_{i-1}=L_{i} \sin \phi_{i}$, where we take $\phi_{i}-\phi_{i-1}=A \sin (\omega t)$ for all $i$. Assuming equal half-link lengths $L$, this equation may be solved exactly, so that $\cos \left(\phi_{i}\right)=|\sin (A \sin (\omega t))|$. The net propulsive force is then given by

$$
F_{n e t}=\frac{N \tau}{4 L}\left(c_{f}-c_{b}\right)|\sin (A \sin (\omega t))|
$$

where $N$ is the number of links in the mechanism, $\tau$ is the torque applied at each joint, and $c_{f}$ and $c_{b}$ are the coefficients of friction for forward and backward motion, respectively. 


\subsection{Three Dimensional Motions}

The rigid structure of each Planar Bender module allows for rotation about the axis which runs along the length of its body (which we call the $z$-axis) without a change in bending due to coupling or sagging. Thus, a rolling motion of a bender about the $z$-axis by an angle $\Theta$ transforms the local coordinate frame by $R_{z, \Theta}$, the rotation matrix about the $z$ axis for that angle.

The Planar Bender is designed for both planar maneuvers and spatial maneuvers in conjunction with a rotational unit. Including a pure rolling-DOF departs from the biological design, and may allow for the use of gaits not found in nature. A gait similar to sidewinding, where bending sections are progressively lifted and placed down, should also be possible. Performing three-dimensional movements will require precise coordination of the bendingDOF with the rolling-DOF, making it necessary to understand how the dynamics of the bending mechanism, and particularly its moments of inertia, change as it bends. A representation of the mechanism, with variables used in the below moment of inertia calculations, is displayed below.

We define the $y$-axis as the axis perpendicular to the plane of bending and the $z$-axis as the one about which the rolling-DOF acts (referring to Fig. 1), and we define $I_{Y, i}$ and $I_{Z, i}$ as the moments of inertia of the $i$ th link about these respective axes. Modeling the links as thin rods, the $y$-axis moments of inertia may be computed recursively as $I_{Y, i}=$ $m_{i} \lambda_{i, y}{ }^{2}+m_{i} h_{i}{ }^{2} / 12$, where $\lambda_{i, y}=\left[\Lambda_{i-1}{ }^{2}+\left(h_{i} / 2\right)^{2}-\Lambda_{i-1} h_{i} \cos \left(\psi_{i-1}\right)\right]^{1 / 2}$ is the distance from the origin to the $i$ th link center and $\Lambda_{i}=\left[\Lambda_{i-1}{ }^{2}+h_{i}^{2}-2 \Lambda_{i-1} h_{i} \cos \left(\psi_{i-1}\right)\right]^{1 / 2}$ is the distance from the origin to the $i$ th joint. We define the angle $\psi_{i}=\theta_{i}-\alpha_{i}$, where $\alpha_{i}=\arcsin \left[\left(h_{i-1} / \Lambda_{i}\right) \sin \left(\psi_{i-1}\right)\right]$, and $\psi_{1}=\theta_{1}$. 


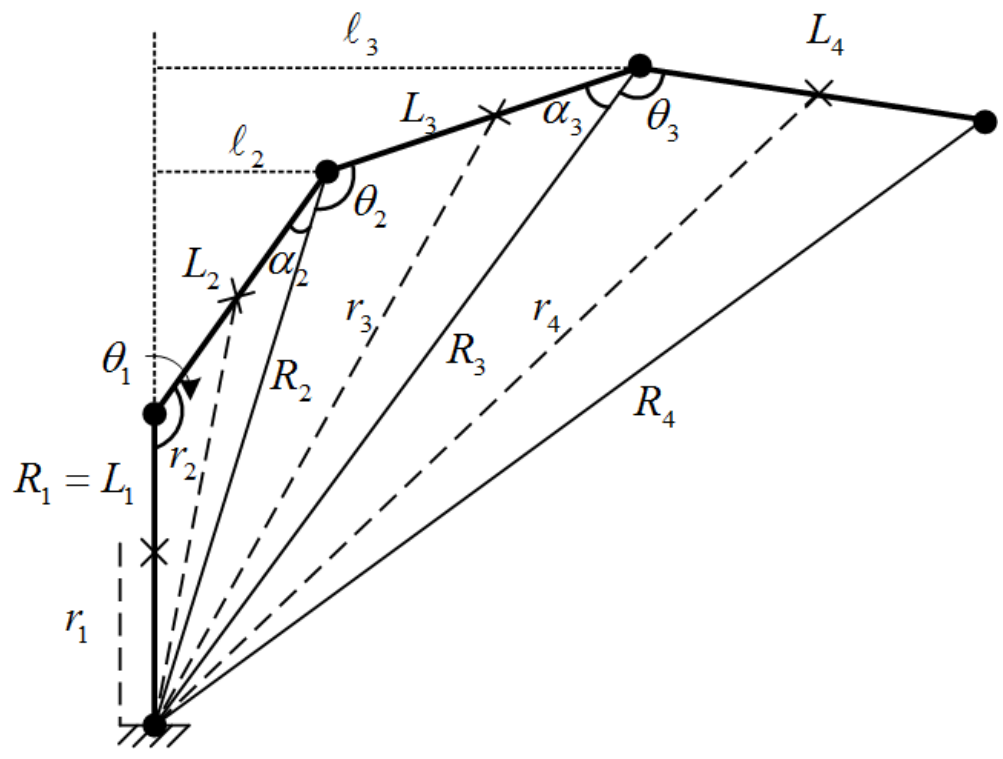

Fig. 27. Position and forward speed of an 8-link snake robot.

The $z$-axis moment of inertia may be modeled by treating each of the links as a solid cylinder of radius $l_{i}$, height $h_{i}$, and mass $M_{i}$. Employing moment of inertia tensor transformations, $I_{Z, i}=1 / 2\left(I_{z, i}+I_{x, i}\right)+1 / 2\left(I_{z, i}-I_{x, i}\right) \cos \left(2 \theta_{i}\right)$, where $I_{z, i}=1 / 2 M_{i} l_{i}^{2}$ is the moment of inertia of each cylinder about its axis and $I_{x, i}=\left(M_{i} / h_{i}\right)\left[l_{i}^{2}\left(h_{i}-\lambda_{i, z}\right) / 4+\left(h_{l}-\lambda_{i, z}\right)^{3} / 3\right]$ is the moment of inertia of each cylinder about a line normal to its surface and passing through its base. Here, $I_{x, i}$ is given by the relation $\lambda_{i, z}=\Sigma_{k=2}^{i}\left|h_{k-1} \sin \left(\theta_{k-1}\right)\right|$. The above expressions for $I_{y}$ and $I_{z}$ were computed numerically, where $M_{i}, h_{i}, l_{i}$, and $\theta_{i}$ were taken to be constant for all $i$. Fig. 28 displays the results of these calculations, normalized by the moments of inertia of the first link, as the angle of the first joint moves from $-60^{\circ}$ to $60^{\circ}$. 


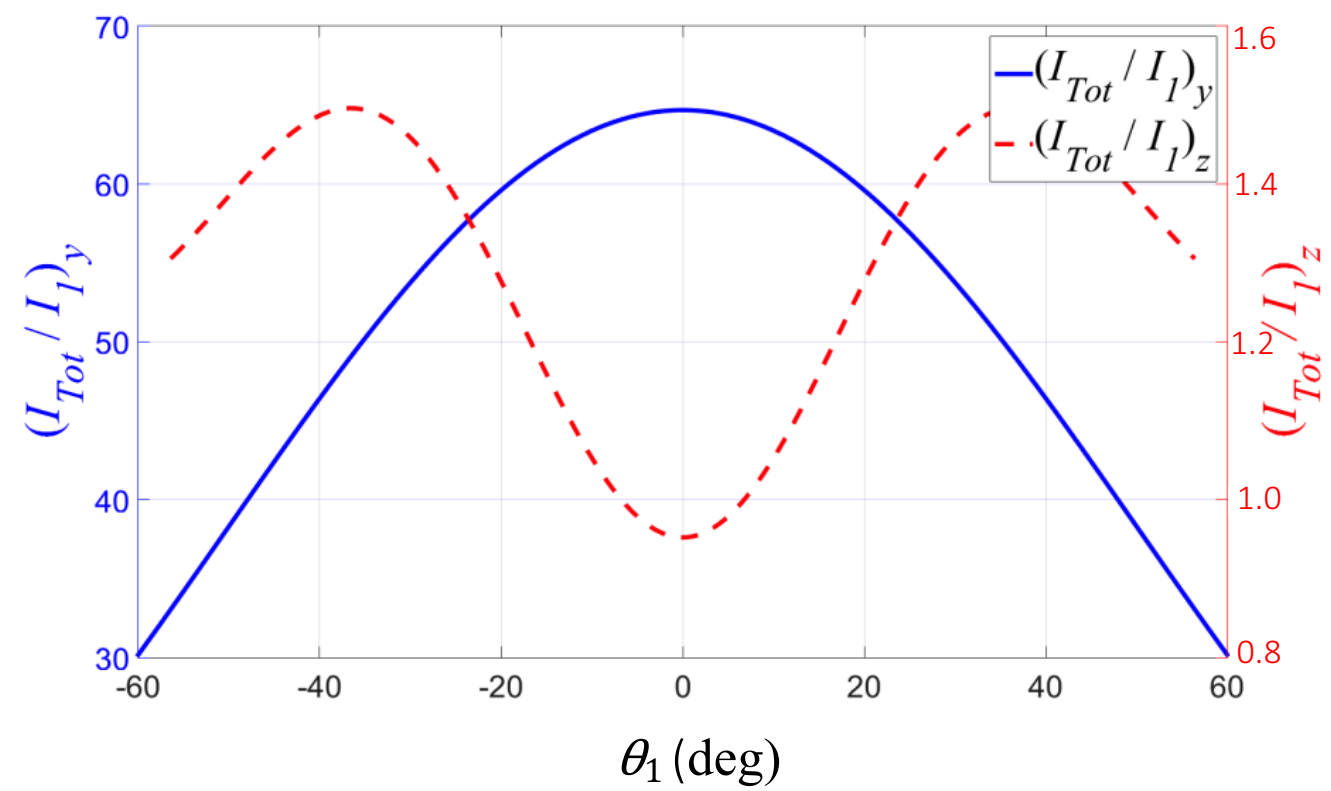

Fig. 28. Modeled Moments of Inertia of the Bending Module

\subsection{Tripedal Configuration}

Three benders may be arranged together at angular displacements of $\gamma=120^{\circ}$ to form a Triped Robot. Since each leg is restricted to a planar bending motion, the other two legs are entirely responsible for stabilizing the motion of that leg perpendicular to this plane. Thus, we may write a s-tability controller in three parts, in which each of the legs is stabilized separately. For each leg, we draw a plane perpendicular to the leg's plane of bending, onto which we project the other two legs, and control the orientation of the legs within that plane. The relative angles between benders are fixed, so the projection of a bender into the plane is given by multiplying its position in its own bending plane by the rotation matrix $R_{\gamma}=\left[\begin{array}{cc}\sin (\gamma / 2) & 0 \\ 0 & 1\end{array}\right]$. Multiply by the inverse matrix $R_{\gamma}^{-1}$ to transform back into the frame of the bender. A feedforward term may be calculated by counteracting the torque that gravity applies on the feet at a given configuration. A PD control law is then 
applied to maintain the foot orientations $\alpha_{i}$ at the desired values. The foot orientation is calculated using the IMU in the head. Locomotion may be achieved by varying the $\alpha_{i}$ sinusoidally:

$$
\alpha_{i, \text { ref }}=A_{i}\left(\sin \left(\omega t+\varphi_{i}\right)+\mid \sin \left(\omega t+\varphi_{i} \mid\right)-\delta_{i}\right.
$$

Setting $\varphi_{2}-\varphi_{1}=\pi$ and $\varphi_{2}=\varphi_{3}$ results in a gait in which a "front leg" and two synchronized "back legs" take turns stepping forward and standing in place. By tuning the values of the amplitudes $A_{i}$ and offsets $\delta_{i}$, a crawling gait may be achieved, in which the front leg moves through a large displacement to provide the primary source of propulsion, while the back legs act as stabilizers, or alternately, two legs propel while one stabilizes. During this motion, all of the feet always maintain contact with the ground, so locomotion requires anisotropic friction on the feet. For this purpose, wedge shaped adapter pieces were designed, which can connect to a bender module via the male-female interface.

Tipping is prevented by ensuring that the center of mass $(\mathrm{CM})$ remains inside the robot's support polygon. As in the above controller, we can simplify the situation by separately viewing the projections of the robot onto the planes perpendicular to each of the three legs. Preventing tipping than reduces to constraining each of the projections of the $\mathrm{CM}$ to remain on the "support line" in between each of the three pairs of adjacent legs. If the projection of the $\mathrm{CM}$ in a particular plane approaches one of the feet, a "tipping controller" acts on the leg so that its projection on that plane extends, reestablishing a stable configuration.

The control action can be made to be continuous by giving the tipping controller the form of a potential well: $\tau_{\min }=a\left(x-x_{\min }\right)^{-b}$ and $\tau_{\max }=a\left(x-x_{\max }\right)^{-b}$ up to the maximum torque, after which point the controller continues to apply its maximum value. Note that we have 
defined positive torque to correspond to inward bending of the leg. Tuning the parameters $a$ and $b$, the potential well can be made arbitrarily steep, to prevent interference with the action of the controller in (37). Each leg applies one $\tau_{\min }$ and $\tau_{\max }$, corresponding to the other two legs, so there are six total tipping controllers.

A multibody dynamic model of one such planar projection was used to validate the stability controller, the crawling gait given by (37), and the tipping controller. In this model, ground was treated as a series of stiff spring dampers, which apply forces at each $\mathrm{CM}$, and forces and torques at each link end. The simulated planar robot succeeded in (a) raising itself from a flat configuration to a standing position, (b) maintaining a standing position using the stability controller, c) crawling forward, and (d) smoothly lowering itself to the ground from a standing position. One of the advantages of this design is that the robot is symmetric with respect to the bending planes of its three legs. Thus, directional changes can be accomplished without any manipulation of leg orientation by simply changing which leg acts as the front leg. 


\section{CHAPTER 8}

\section{EXPERIMENTAL SETUP AND RESULTS}

This section presents the results of experimental studies of force control, locomotion, and obstacle interaction in the snake robot prototype introduced in Sec. 4.1.

\subsection{Force Control}

The force controller described in Sec. 6.6 was tested in a single module in two configurations, $\theta_{1}=0^{\circ}$ and $\theta_{1}=30^{\circ}$, for three desired force outputs, $10 \mathrm{~N}, 15 \mathrm{~N}$, and $20 \mathrm{~N}$. A single module was placed horizontally, so that gravitational effects could be ignored, and allowed to reach the desired configuration unimpeded. The last link of the module was then placed parallel to an MLP-25 compression load cell rated for $44.5 \mathrm{~N}$, and the module's base was fixed to ground. At $\theta_{1}=0^{\circ}$ and $10 \mathrm{~N}$ desired force, the model in Sec. 6.6 predicts a required pulley angular displacement of $\Delta \theta_{\mathrm{P}}=35.3^{\circ}$. In practice, $\Delta \theta_{\mathrm{P}}$ had to be adjusted approximately $6 \%$ above this value to achieve the desired force output, possibly as a result of frictional effects or errors in the kinematic constraints. This correction multiplier was held constant in all subsequent experiments. In accordance with the model in Sec. 6.6, in the $\theta_{1}=30^{\circ}$ configuration, the robot commands a $\Delta \theta_{\mathrm{P}}$ approximately 1.4 times the value required for the $\theta_{1}=0^{\circ}$ case. The results of these experiments are shown in Fig. 29, after application of a locally weighted linear regression smoothing. Note that the load cell used in these experiments has a settling time of over a second, which causes the force measurements to decrease slightly after the applied force has plateaued. The results in Fig. 29 demonstrate that the bending module is able to successfully control the perpendicular 
component of the end effector force by controlling the pulley's angular position, with less than $5 \%$ error in all but one of the six trials.
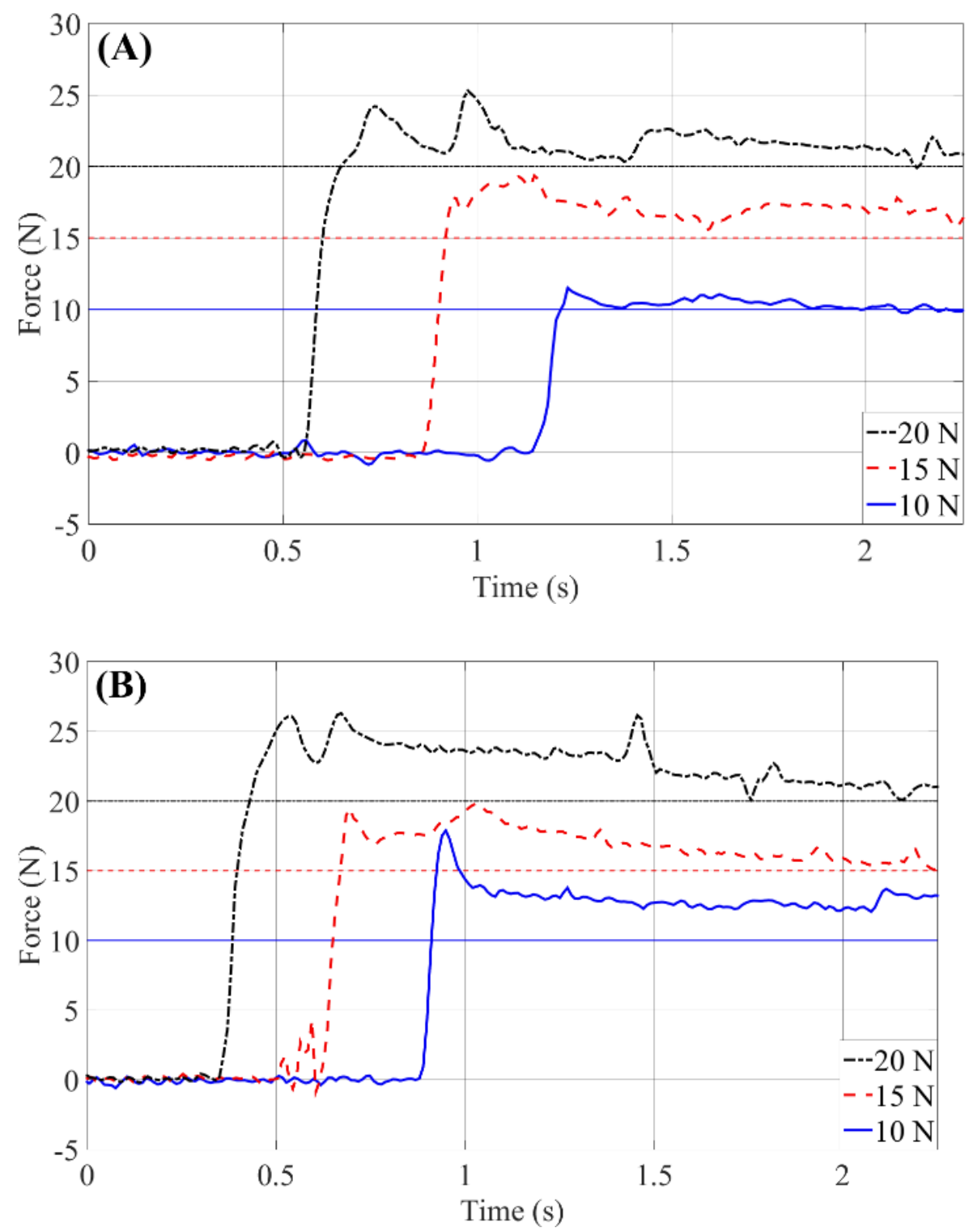

Fig. 29. Force control experiments for (A) Straight configuration $\left(\theta_{1}=0^{\circ}\right)$ and (B) Curved configuration $\left(\theta_{1}=30^{\circ}\right)$. 


\subsection{Locomotion and Steering}

As discussed in [49], the controller outlined in Fig. 27 was tested in simulation using the MBD model from Sec. 6.6. In the prototype, the feedback linearization is removed, the path follower is replaced by manual steering, and the output of the controller is cascaded into the servomotor's internal PID loop.

Planar locomotion and steering were tested in the prototype by tracking its trajectory on floor tiles over roughly four periods of oscillation, starting from a straight configuration and with $\alpha=2.45 \mathrm{rad}, \delta=1.27 \mathrm{rad}$, and $\omega=3.77 \mathrm{~Hz}$. The front link of the snake was labeled with a blue marker and the back link with a red marker, as shown in the snapshots in Fig. 30. (Locomotion seems to be more effective when the "head" module is dragged behind the snake rather than pushed ahead of it.)

A 12.3 megapixel camera positioned at a height of approximately two meters and facing the ground was used to record the motion of the snake. These videos were sampled at 10 $\mathrm{Hz}$ and scaled using the known dimensions of the tiles, after which the centroids of each marker were tracked. The resulting trajectories are shown in Fig. 31, for values of $\theta_{0}$ between $-0.15 \mathrm{rad}$ and $0.15 \mathrm{rad}$.

The prototype is capable of traveling at speeds of about 0.07 body lengths per second, comparable to the speed of serpentine locomotion in some large biological snakes [53], but more than an order of magnitude slower than the fastest snakes [4]. The prototype appears to have a slight bias to the left and exhibits higher amplitude oscillations during right turning than left turning, most likely on account of asymmetries in the cable constraints. However, an orientation offset of this size can also occur in simulation due to initial conditions, and is easily corrected by actively controlling $\theta_{0}$. 


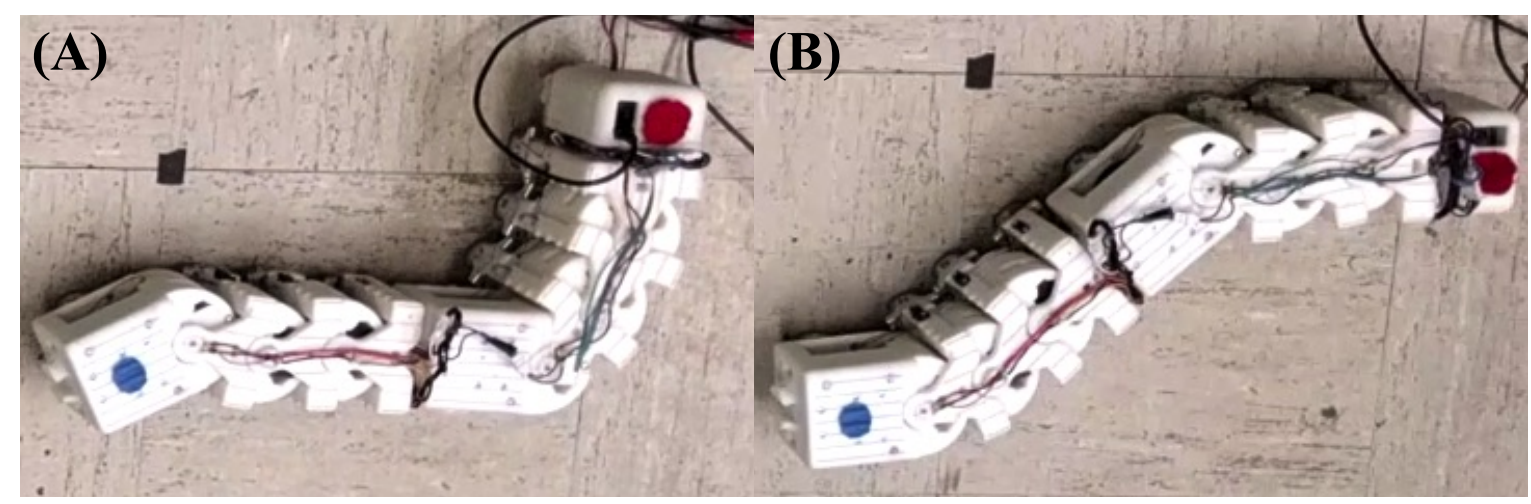

Fig. 30. Snapshots of the snake robot executing a counterclockwise turn, showing a half period of oscillation between (A) and (B). The black marker in the top left corner provides a point of reference.

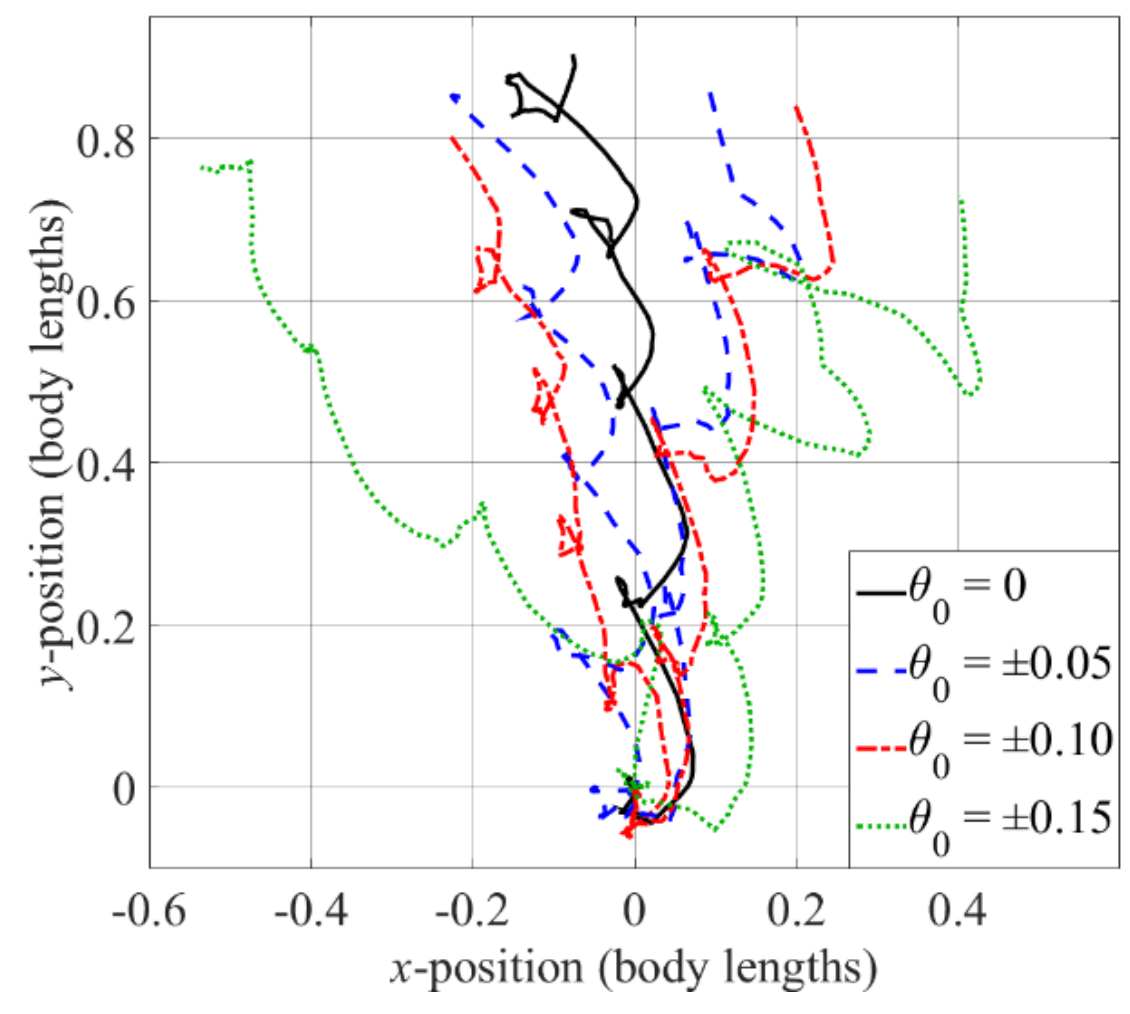

Fig. 31. Trajectories of the front link of the snake robot over approximately four oscillation periods, for various values of $\theta_{0}$. (Positive values correspond to clockwise turning). 


\subsection{Obstacle Interaction}

The obstacle interaction procedure of (35) was tested experimentally by recording the trajectory of the snake robot as it moved past a circular, $5 \mathrm{~kg}$ cast iron plate, with a radius of approximately $8 \mathrm{~cm}$. The robot was started in a straight configuration, flush with the obstacle and tangent to it, at an angle of approximately $45^{\circ}$ from the global $y$-axis. The robot was then commanded to move forward, with $\theta_{0}=0$ and the other controller parameters set at the same values as in the locomotion experiment in Sec. 8.2. The experiment was repeated for three values of $k_{\mathrm{obs}}$, the results of which are displayed in Fig. 32. In these experiments, the front and back of the snake are tracked as in Sec. 8.2 and the angle that the vector between the red and blue markers forms with the global $y$-axis is taken as a good approximation for the snake's global orientation. The initial and period-averaged final orientations of the snake are denoted in the figure by arrows.

Without the controller in (35) (i.e. $k_{\mathrm{obs}}=0$ ), the snake is observed to rotate about the obstacle by the following process. The front module contacts the obstacle first and induces a moment about the snake's CM that rotates the robot away from the obstacle. However, this movement brings the back module into contact with the obstacle, and a moment is then induced in the opposite direction. At this point, for an obstacle of this size, the front module has moved past the obstacle and only the back module continues to contact it. The snake is then progressively rotated, but the curvature of its trajectory is low enough that the point of contact with the obstacle moves down the length of the back module until the snake eventually loses contact with the obstacle and moves past it.

Thus, two competing tendencies determine the amount of rotation induced by an obstacle: the magnitude of the moment produced by each impact and the amount by which 
the point of contact moves down the length of the snake between impacts. In the experiment in Fig. 32, the case $k_{\mathrm{obs}}=0$ results in about a $168^{\circ}$ change in orientation before contact with the obstacle is lost. Increasing $k_{\text {obs }}$ to 1.33 , the snake tends to bend more in the direction of contact, resulting in an orientation change of about $221^{\circ}$ over the same period. On the other hand, a negative value of $k_{\text {obs }}$ effectively offsets obstacle induced rotation by rotating the reference heading in the opposite direction. The snake can thus be made to push off or continue past an obstacle, as in the case $k_{\mathrm{obs}}=-2$ in the figure, where the snake continues on with little change to its initial heading (only about $17^{\circ}$ ). Note however, that this behavior is highly dependent on the size and shape of the obstacle.

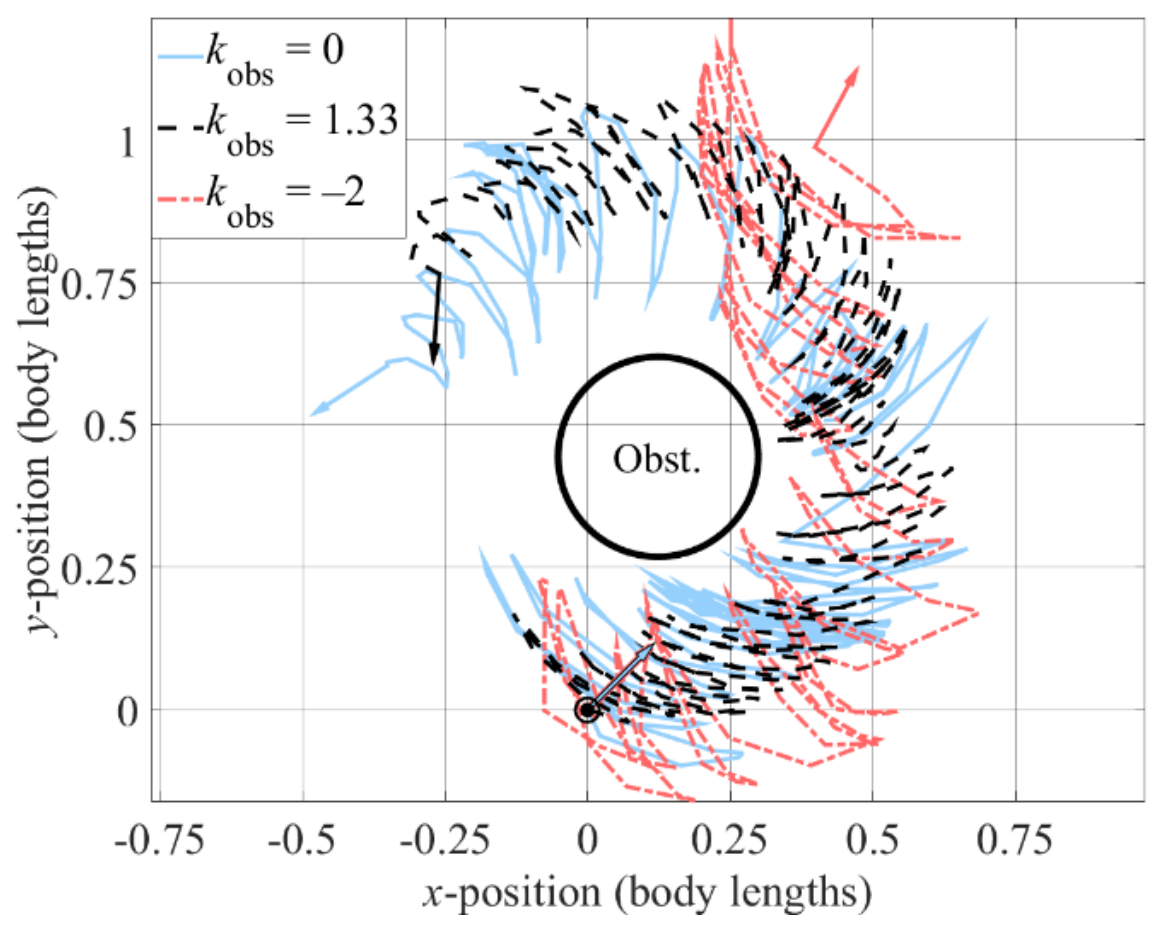

Fig. 32. Trajectories of the back link of the snake robot during interaction with a circular obstacle. Initial position is denoted by two concentric circles at $(0,0)$. 


\section{CHAPTER 9}

\section{CONCLUSION \& FUTURE WORK}

\subsection{Summary}

This paper presented the development of a cable-driven snake robot with coupled joints, which incorporates series elastic elements as a means of producing automatic obstacle interaction behavior. A simplified model of serpentine locomotion was developed and used to analyze how the locomotive efficiency of a snake robot is related to the number of links it contains. A control scheme was devised for a snake robot comprised of coupled linkages and combined with a feedback linearization of the joint dynamics. These ideas were tested in simulation studies and experiments with a prototype.

\subsection{Future Work}

Whereas the cable-actuated module presented in this paper can approximate planar curvature profiles, the antagonistic cable routing scheme of the present design is not well suited to three-dimensional motions. With more sophisticated cable routing, additional DOFs may be possible in a single module, enabling more natural and versatile locomotion. The use of the bending module as a modular reconfigurable robotic platform is also being explored, and a second "spinner" module, which allows for rotation of the snake about its principal axis, has been designed to allow for three-dimensional motions such as sidewinding. Decoupled "bending" and "spinning" degrees of freedom do not exist in biological snakes and may facilitate locomotion gaits not found in nature. 


\section{REFERENCES}

[1] S. Hirose, "Biologically Inspired Robots (Snake-like Locomotors and Manipulators)," Oxford Univ. Press, 1993.

[2] P. Liljebäck, K. Y. Pettersen, Ø. Stavdahl, and J. T. Gravdahl, "Fundamental properties of snake robot locomotion," IEEE/RSJ Int. Conf. Intell. Robot. Syst., no. May, pp. 2876-2883, 2010.

[3] J. Gray, "The Mechanism of Locomotion in Snakes," J. Exp. Biol., vol. 23, no. 2, 1946.

[4] D. L. Hu, J. Nirody, T. Scott, and M. J. Shelley, "The mechanics of slithering locomotion.," Proc. Natl. Acad. Sci. U. S. A., vol. 106, no. 25, pp. 10081-10085, 2009.

[5] P. Liljebäck, K. Y. Pettersen, Ø. Stavdahl, and J. T. Gravdahl, Snake Robots: Modeling, Mechatronics, and Control. Springer, 2013.

[6] A. Degani, H. Choset, B. Zubiate, T. Ota, and M. Zenati, "Highly Articulated Robotic Probe for Minimally Invasive Surgery," Conf. Proc. IEEE Eng. Med. Biol. Soc., vol. 2006, no. 1.642343E6, pp. 4167-4172, 2006.

[7] J. Burgner-Kahrs, D. C. Rucker, and H. Choset, "Continuum Robots for Medical Applications: A Survey," IEEE Trans. Robot., vol. 31, no. 6, pp. 1261-1280, 2015.

[8] A. A. Transeth, K. Y. Pettersen, and P. Liljebäck, "A survey on snake robot modeling and locomotion," Robotica, vol. 27, no. March 2009, p. 999, 2009.

[9] S. Hirose and H. Yamada, "Snake-like robots: Machine design of biologically inspired robots," IEEE Robot. Autom. Mag., vol. 16, no. 1, pp. 88-98, 2009.

[10] C. Wright et al., "Design and architecture of the unified modular snake robot," 
Proc. - IEEE Int. Conf. Robot. Autom., pp. 4347-4354, 2012.

[11] D. Rollinson, A. Buchan, and H. Choset, "Virtual chassis for snake robots:

Definition and applications," Adv. Robot., vol. 26, no. 17, pp. 1-22, 2012.

[12] M. Tesch et al., "Parameterized and Scripted Gaits for Modular Snake Robots," Adv. Robot., vol. 23, pp. 1131-1158, 2009.

[13] G. Granosik, M. G. Hansen, and J. Borenstein, "The OmniTread serpentine robot for industrial inspection and surveillance," Ind. Robot An Int. J., vol. 32, no. 2, pp. 139-148, 2005.

[14] J. Borenstein, G. Granosik, and M. Hansen, "The OmniTread serpentine robot Design and field performance," Proc. SPIE - Int. Soc. Opt. Eng., vol. 5804, pp. $324-332,2005$.

[15] S. Murata, E. Yoshida, A. Kamimura, H. Kurokawa, K. Tomita, and S. Kokaji, “M-TRAN : Self-Reconfigurable Modular Robotic System,” Trans. Mechatronics, vol. 7, no. 4, pp. 431-441, 2002.

[16] M. Yim, D. G. Duff, and K. D. Roufas, "PolyBot: a modular reconfigurable robot," Proceedings of the 2000 IEEE International Conference on Robotics \& Automation, vol. 1. pp. 514-520, 2000.

[17] H. Yamada, S. Chigisaki, M. Mori, K. Takita, K. Ogami, and S. Hirose, "Development of amphibious snake-like robot ACMR5," Proc. Int. Symp. Robot. ISR '05, 2005.

[18] B. Ouyang, Y. Liu, and D. Sun, "Design of a three-segment continuum robot for minimally invasive surgery," Robot. Biomimetics, vol. 3, no. 1, pp. 1-4, 2016.

[19] W. Mosauer, “On the locomotion of snakes,” Science (80-. )., no. 76, pp. 583-585, 
1932.

[20] J. Gasc, “Axial musculature,” Biol. Reptil., vol. 11, 1981.

[21] M. Mori and S. Hirose, "Development of active cord mechanism ACM-R3 with agile 3D mobility," Proc. IEEE/RSJ Int. Conf. Intell. Robot. Syst., vol. 3, pp. $1552-1557,2001$.

[22] M. J. Baum, A. E. Kovalev, J. Michels, and S. N. Gorb, “Anisotropic friction of the ventral scales in the snake lampropeltis getula californiae," Tribol. Lett., vol. 54, no. 2, pp. 139-150, 2014.

[23] B. C. Jayne, "Mechanical behaviour of snake skin,” J. Zool., vol. 214, no. 1, pp. $125-140,1988$.

[24] H. Marvi, G. Meyers, G. Russell, and D. L. Hu, "Scalybot: A snake-inspired robot with active control of friction," ASME 2011 Dyn. Syst. Control Conf. Bath/ASME Symp. Fluid Power Motion Control. DSCC 2011, vol. 2, pp. 443-450, 2011.

[25] M. M. Serrano, A. H. Chang, G. Zhang, and P. A. Vela, "Incorporating frictional anisotropy in the design of a robotic snake through the exploitation of scales," Proc. - IEEE Int. Conf. Robot. Autom., vol. 2015-June, no. June, pp. 3729-3734, 2015.

[26] W. Saab, A. Kumar, and P. Ben-Tzvi, "Design and Analysis of a Miniature Modular Inchworm Robot," ASME 2016 Int. Des. Eng. Tech. Conf. Comput. Inf. Eng. Conf., p. DETC2016-59386, 2016.

[27] B. L. Conrad, J. Jung, R. S. Penning, and M. R. Zinn, "Interleaved continuum-rigid manipulation: An augmented approach for robotic minimally-invasive flexible catheter-based procedures," Proc. - IEEE Int. Conf. Robot. Autom., pp. 718-724, 
2013.

[28] Z. Li and R. Du, "Design and analysis of a bio-inspired wire-driven multi-section flexible robot: Regular paper," Int. J. Adv. Robot. Syst., vol. 10, 2013.

[29] S. Takaoka, H. Yamada, and S. Hirose, "Snake-like active wheel robot ACM-R4.1 with joint torque sensor and limiter," IEEE Int. Conf. Intell. Robot. Syst., pp. 1081-1086, 2011.

[30] M. G. Catalano, G. Grioli, E. Farnioli, a. Serio, C. Piazza, and a. Bicchi, "Adaptive synergies for the design and control of the Pisa/IIT SoftHand," Int. J. Robot. Res. , vol. 33, no. 5, pp. 768-782, 2014.

[31] R. H. Grebenstein et al, 2011. C. Grebenstein, A. Albu-Schaffer, T. Bahls, M. Chalon, O. Eiberger, W. Friedl, R. Gruber, S. Haddadin, U. Hagn, “The dlr hand arm system," Robot. Autom., 2011.

[32] C. B. A. Gimenez A. Jardon, "Diseño y simulación de un actuador de rigidez variable," An. Ing. Mecánica Rev. la Asoc. Española Ing. Mécanica, 2012.

[33] P. Liljeback, K. Y. Pettersen, O. Stavdahl, and J. T. Gravdahl, "Compliant control of the body shape of snake robots," Proc. - IEEE Int. Conf. Robot. Autom., pp. 4548-4555, 2014.

[34] G. A. Pratt and M. M. Williamson, "Series elastic actuators," IEEE/RSJ Int. Conf. Intell. Robot. Syst. 'Human Robot Interact. Coop. Robot., vol. 1, no. 1524, pp. 399-406, 1995.

[35] M. Ahmed and M. M. Billah, "Smart Material-actuated Flexible Tendon-based Snake Robot," Int. J. Adv. Robot. Syst., p. 1, 2016.

[36] S. Ma, "Analysis of snake movement forms for realization of snake-like robots," 
Robot. Autom. 1999. Proceedings. 1999 IEEE Int. Conf., vol. 4, no. May, pp. 3007-3013 vol.4, 1999.

[37] F. L. Chernousko, "Snake-like locomotions of multilink mechanisms," JVC/Journal Vib. Control, vol. 9, no. 1-2, pp. 235-256, 2003.

[38] E. Rezapour and P. Liljebäck, "Path following control of a planar snake robot with an exponentially stabilizing joint control law," IFAC Proc. Vol., vol. 8, no. PART 1, pp. 28-35, 2013.

[39] P. Liljebäck, I. U. Haugstuen, and K. Y. Pettersen, "Experimental investigation of a path following controller for planar snake robots," 11th Int. Conf. Control. Autom. Robot. Vision, ICARCV 2010, no. December, pp. 2325-2332, 2010.

[40] P. Liljebäck and K. Y. Pettersen, "Waypoint guidance control of snake robots," Proc. - IEEE Int. Conf. Robot. Autom., no. 7491, pp. 937-944, 2011.

[41] P. Liljebäck, K. Y. Pettersen, and Ø. Stavdahl, "Modelling and control of obstacleaided snake robot locomotion based on jam resolution," Proc. - IEEE Int. Conf. Robot. Autom., no. 7491, pp. 3807-3814, 2009.

[42] P. Racioppo, W. Saab, and P. Ben-Tzvi, "Design and Analysis of a Reduced Degree of Freedom Modular Snake Robot," Int. Des. Eng. Tech. Conf. Comput. Inf. Eng. Conf., 2017.

[43] G. A. Pratt and M. M. Williamson, "Series Elastic Actuators," in Proceedings 1995 IEEE/RSJ International Conference on Intelligent Robots and Systems., 1995.

[44] D. Rollinson, S. Ford, B. Brown, and H. Choset, "Design and Modeling of a Series Elastic Element for Snake Robots,” ASME 2013 Dyn. Syst. Control Conf., p. 
V001T08A002, 2013.

[45] D. Rollinson et al., "Design and architecture of a series elastic snake robot," 2014 IEEE/RSJ Int. Conf. Intell. Robot. Syst. IROS 2014, Sept. 14, 2014 - Sept. 18, 2014, no. Iros, pp. 4630-4636, 2014.

[46] P. Racioppo and P. Ben-Tzvi, "Design and Analysis of a Reduced Degree of Freedom Snake Robot," Unpublished.

[47] E. Haug, "Computer-Aided Kinematics and Dynamics of Mechanical Systems, Volume I: Basic Methods," Massachusetts: Allyn and Bacon. 1989.

[48] E. J. Haug and J. S. Arora, Applied Optimal Design. New York: Wiley Interscience, 1979.

[49] P. Racioppo and P. Ben-Tzvi, "Modeling and Control of a Cable Driven Modular Snake Robot," IEEE Conf. Control Technol. Appl., 2017.

[50] P. Liljeback, K. Y. Pettersen, O. Stavdahl, and J. T. Gravdahl, "Controllability and stability analysis of planar snake robot locomotion," IEEE Trans. Automat. Contr., vol. 56, no. 6, pp. 1365-1380, 2011.

[51] H. Date and Y. Takita, "Adaptive Locomotion of a Snake Like Robot Based on Curvature Derivatives," pp. 3554-3559, 2007.

[52] D. Rollinson, K. V. Alwala, N. Zevallos, and H. Choset, "Torque control strategies for snake robots," IEEE Int. Conf. Intell. Robot. Syst., no. Iros, pp. 1093-1099, 2014.

[53] H. Marvi, J. Bridges, and D. L. Hu, "Snakes mimic earthworms: Propulsion using rectilinear travelling waves," J. R. Soc. Interface, vol. 10, no. 84, 2013. 